\title{
THE DETERMINATION OF SENSORY DEFICITS IN CHILDREN WITH HEMIPLEGIC CEREBRAL PALSY
}

\author{
$\star \star \star \star \star *$ \\ JASMINE COOPER, B.SC. \\ SCHOOL OF PHYSICAL AND OCCUPATIONAL THERAPY, \\ MCGILL UNIVERSITY, \\ MONTREAL, QUEBEC. \\ Date: March 17, 1992.
}

A thesis submitted to the faculty of Graduate Studies and Research in partial fulfillment of the requirements of the degree of Master of Rehabilitation Science.

(c) Jasmine F. Cooper, 1992. 


\begin{abstract}
Cerebral palsy is a non-progressive disorder associated with brain injury, defect, or disease, of early onset. Emphasis is placed on the more easily observable motor deficits, with any underlying sensory deficits often being overlooked. Existing sensory assessments have either been standardized on an adult population or for a paediatric population without significant neuromotor imparrment. Thus, there is a need to formulate a standardized sensory assessment battery that may be used to evaluate physically handicapped children. A review of the literature highlights the necessity to evaluate the presence and extent of sensory dysfunction in school-age hemiplegıc children, using a standardized clinical sensory assessment battery, as well as somatosensory evoked potentials.

A reliable sensory battery, which assesses 5 sensory modalities, was formulated and normative data for school-age children was derived. Sensory function was evaluated in 9 hemiplegic children (419 years) and 18 healthy age-matched controls. Significant bilateral sensory deficits were documented in hemiplegic children. Thus a sensory assessment should be an integral part of the evaluation of a child with hemiplegia.
\end{abstract}




\section{SOMMAIRE}

La paralysie cérébrale est un désordre non-progressıf causé par un accident cérébral qui se produit assez tôt dans le développement L'emphase est placée sur les déficits moteurs qui sont plus facilement observables que les déficits sensitifs, bien que ces derniers existent et sont souvent mis de côté. Des données relatives aux troubles sensitifs ont été accumulées solt pour les adultes, solt pour les enfants sans problèmes moteurs importants 11 serait cependant important de formuler une évaluation sensitive standardisée utilisable dans l'evaluation d'enfants handicapés physiquement. En revisant la littérature, on s'aperçoit de la nécessité d'évaluer la présence et l'éntendue des dysfonctions sensitives chez les enfants hémiplégiques d'âge scolaıre en utılısant un mode d'évaluation standard comprenant différents test aınsı que les potentiels évoqués somesthésiques

Différents tests sensitifs fiabies furent exécutés; ceux-cI évaluaient 5 modalités sensitives et les valeur normales furent obtenues chez des enfants d'age scolaire L'évaluation sensitive de 9 enfants hémiplégıques ( $4-19$ and) et 18 enfants sains fut obtenue De sérieux déficits sensitifs bilatéraux furent documentés chez les enfants hémiplégiques. Donc, l'évaluation sensitive devrait toujours faire partie integrante de l'evaluation globale d'enfants hémiplégiques. 


\section{STATEMENT OF AUTHORSHIP:}

I certify that 1 am the primary author of the manuscript contained in this thesis. I ciaim full responsibility for the content and style of the text included herein. 


\section{Abbreviations.}

$\begin{array}{ll}\text { 2pd } & \text { 2-point discrimination } \\ \text { BAEP } & \text { Brainstem Auditory Evoked Potentials } \\ \text { CII } & \text { Second Cervical Vertebra } \\ \text { Cc } & \text { Contralateral Parietal Scalp } \\ \text { CP } & \text { Cerebral Palsy } \\ \text { CT } & \text { Computed Tomography } \\ \text { EP } & \text { Erb's Point } \\ \text { Fz } & \text { Midline of Upper Forehead } \\ \text { ICC } & \text { Intraclass Correlation Coefficient } \\ \text { IF } & \text { Index Finger } \\ \text { K } & \text { Kappa Coefficient } \\ \text { LF } & \text { Little Finger } \\ \text { LH } & \text { Left Hemiplegic } \\ \text { m2pd } & \text { Moving Two-Point Discrimination } \\ \text { MAP } & \text { Miller Assessment For Preschoolers } \\ \text { MRI } & \text { Magnetic Resonance Imaging } \\ \text { QNST } & \text { Quick Neurological Screening Test } \\ \text { RH } & \text { Right Hemiplegic } \\ \text { S I } & \text { Somesthetic Sensory Area I } \\ \text { S II } & \text { Somesthetic Sensory Area II } \\ \text { SCSIT } & \text { Southern California Sensory Integration Tests } \\ \text { S.d. } & \text { Standard Deviation } \\ \text { SEP } & \text { Somatosensory Evoked Potentials } \\ \text { TH } & \text { Thumb } \\ \text { VEP } & \text { Visual Evoked Potentials } \\ \text { VPL } & \text { Ventroposteriolateral nucleus } \\ & \end{array}$


Table of contents

PREFACE

ACKNOWLDGEMENTS

1. LITERATURE REVIEW.

1.1 Definition and Classification of Cirebral Palsy -

1.2 Somatosensory System $\bullet \quad \bullet \quad \cdot \quad \cdot 5$

1.3 Sensory Tests $\bullet \quad \bullet \quad \bullet \quad \bullet \quad \bullet \quad \bullet \quad \cdot 9$

1.3.1 Sensibility Tests. $\bullet \quad \cdot \quad \cdot \quad \cdot 9$

1.3.2 Adult Sensory Tests • $\quad$ • $\quad$ - 12

1.3.3 Paediatric Sensory Tests • • • 12

1.3.4 Somatosensory Evoked Potentials • • 15

1.4 Sensory Testing in Static Encephalopathy - $\quad$ - 17

1.4.1 Clinical Sensory Testing in Cerebral Palsy 17

1.4.2 Neurophysiological Testing (SEP) In

Static Encephalopathy • • • • • 23

1.5 Summary of Literature Review. $\quad$ - $\quad 29$

2. OBJECTIVES •

3. METHODOLOGY •

3.1 Subjects $\bullet \quad \bullet \quad \bullet \quad \cdot \quad \cdot \quad \cdot \quad \cdot 032$

3.1.1 Inclusion Criteria $\bullet \quad \bullet \quad \bullet \quad \bullet \quad 32$

3.2 Controls $\bullet \quad \cdot \quad \cdot \quad \cdot \quad \cdot \quad \cdot \quad \bullet \quad 32$

3.3 Experimental Design $\bullet \quad \cdot \quad \cdot \quad \cdot \quad \cdot 33$

3.3.1 Procedures Followed • $\bullet \quad \cdot \quad \cdot 33$

3.4 Sensory-Motor Assessments • $\quad$ • $\quad$ - 34

3.4.1 Standardization/Reliability/Validity - 34

3.4.2 Motor Assessment • • • • 35

3.4.3 Neurological Examination • • • 35

3.4.4 Somatosensory Evoked Potentials • • 36

3.4.5 Sensory Assessment • • • • 37

3.5 Statistics $\bullet \quad \bullet \quad \bullet \quad \bullet \quad \bullet \quad \bullet \quad \bullet \quad 38$

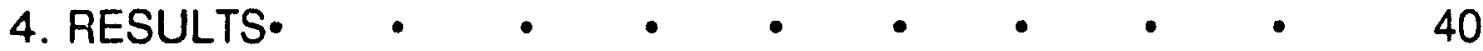

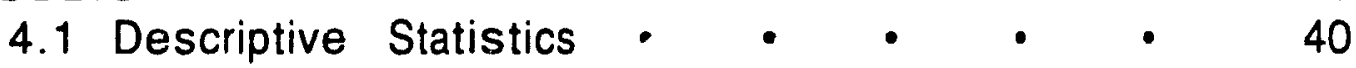

4.1 .1 Controls $• \quad \cdot \quad \cdot \quad \cdot \quad \cdot \quad \cdot 40$

4.1 .2 Subjects $\cdot$ - $\quad \cdot \quad \cdot \quad \cdot \quad \cdot 40$

4.2 Normative Data $\quad \cdot \quad \cdot \quad \cdot \quad \cdot \quad \cdot \quad \cdot 40$

4.2.1 Motor Assessment Battery In The
Control Group • . . . . 41 
4.2.2 Neurological Examination In The

Control Group • • • • • •

4.2.3 Scmatosensory Evoked Potentials In The

Control Group • • • • • •

4.2.4 Sensory Assessment Battery In The

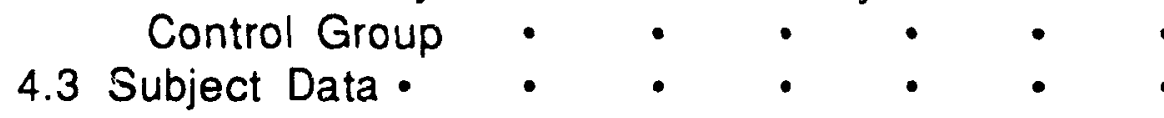

42

4.3.1 Motor Assessment Battery In The

Experimental Group • • • • •

4.3.2 Neurological Examination In The

Experimental Group

4.3.3 Somatosensory Evoked Potentials In The

Experimental Group • $\quad$ •

4.3.4 Sensory Assessment Battery In The

Experimental Group • • • •

4.4 Reliability

5. DISCUSSION

5.1 Formulation of a Reliable Paediatric Test Battery for Children with Neuromotor Deficits - •

5.2 Evaluation of Sensory Function In Hemiplegic Children Using Clırical and Electrophysiological

Techniques

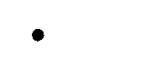

5.3 Relationship between Motor and Sensory Function

in Hemiplegic Children

5.4 Conclusions .

TABLES

FIGURES

APPENDICES•

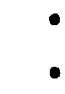




\section{List of tables}

1. Descriptive statistics 71

2. Descriptive statistics 71

3. Somatosensory evoked potentia! control values 72

4. Pressure sensitivity control values 73

5. Moving two-point discrimination control values 74

6. Stereognosis c!ntrol values 74

$\begin{array}{ll}7 . & \text { Proprioception control values } \\ & 75\end{array}$

8 Directionality control values 75

9. Grasp pattern scores of hemiplegic children 76

10. Neurological examination results of hemiplegic children 76

11. CT scan and MRI findings in hemiplegic children 77

12. Somatosensory evoked potential recordings on the 78 affected side in hemiplegic children

13 P values based on sensory function of the affected 79 side of subjects as compared to the non-dominant side of controls

14. $P$ values based on sensory function of the 'non-affected' side of subjects as compared to the dominant side of controls

15. $P$ values based on sensory function of the affected side of subjects as compared to their 'non-affected' side

16. Number of sensory modalities imparred on the affected 80 hand of hemiplegic children

17. Number of sensory modalities impaired on the 'non- 80 affected' hand of hemiplegic children

18. Sensory involvement on the affected hand of hemiplegic 81 children

19 Sensory involvement on the 'non-affected' hand of hemiplegic children

20. Summary of results of the experimental group 82

21. Reliability of the non-dominant/affected hand as 83 measured by the Kappa coefficient

22. Reliability of the dominant/'non-affected' hand as 84 measured by the Kappa coefficient

23. Reliability using the intraclass correlation coefficient 85 on the non-dominant/affected hand

24. Reliability using the intraclass correlation coefficient 85 on the dominant/'non-affected' hand 


\section{List of figures}

Figure 1: Scatter diagram of control values of the grip 86 strength of the non-dominant hand plotted as a function of age.

Figure 2: Scatter diagram of control values of the grip strength of the dominant hand plotted as a function of age.

Figure 3: Scatter diagram of control values of N13-N20 interwave latencies plotted as a function of age.

Figure 4: SEP waveform recordings of a child with left hemiplegia.

Figure 5: Mean pressure sensitivity thresholds of the thumb on the non-dominant/affected hand.

Figure 6: Mean pressure sensitivity thresholds of the dominant/'non-affected' hand.

Figure 7: Mean m2pd thresholds of the non-dominant affected hand

Figure 8: Mean stereognosis scores of the non-dominant/ affected and dominant/'non-affected' hands.

Figure 9: Mean proprioception scores of the non-dominant/ affected hand.

Figure 10: Mean directionality scores of the non-dominant/ affected hand. 


\section{PREFACE}

Cerebral Palsy (CP) is defined as a non-progressive motor disorder due to brain injury, defect, or disease, of early onset (Clark and Allen, 1985). Emphasis has been placed on the more easily observable motor deficits with any underlying sensory deficits often being overlooked. Several studies in the 1950's and 1960's suggested that the incidence of sensory deficits in CP children was quite high (Hohman et al, 1958, Kenney, 1963; Tachdjian and Minear, 1958; Tizard et al, 1954) At least $40 \%$ and up to $73 \%$ of spastic hemiplegic children were found to have sensory impairment (Jones, 1976; Kenney, 1963, Monfraix et al, 1961; Tachdjian and Minear, 1958) The most commonly identified sensory deficits were astereognosis, impaired two-point discrimination and impaired proprioception However these results need to be verified due to the many methodological and statistical errors which exist in these studies Thus, it can be seen that effective evaluation and treatment of children with CP should include sensory assessment due to the high prevalence of sensory deficits in this population

The existıng sensory assessments have either been standardized on an adult population or on a paediatric population with the necessary motor skills to perform the test (Dannenbaum and Dykes, 1988). Review of the literature thus highlights the need to formulate a standardized sensory assessment battery that can be used for children with neuromotor deficits.

Somatosensory Evoked Potentials (SEP) are electrical manifestations of the brain's reception of and response to the stimulation of the somatosensory system (Chiappa, 1990). SEP are a non-noxious, non-Invasive method of evaluating the integrity of the dorsal column-medial lemniscal system. They have been shown to have a high correlation with the severity of neurological impairment, magnitude of sensory deficits present and level of functional disability in an adult stroke population (Karnaze et al, 1987; Larson et al, 1966 Liberson, 1966; Zeman and Yiannikas, 1989).

The dorsal column-medial lemniscal system and parietal cortex mediate sensations of proprioception, two-point discrimination, 
point localization, stereognosis, positıon sense, kınesthesia and directionality (Carlson, 1981; Dykes, 1982; Roland, 1987; Seal, 1989). Thus it would be of interest to test these sensations clinically and compare the results obtained to SEP findings in children sustaining brain injury. It is as yet unclear whether SEP measure the integrity of specific sensory modalities

Hence this study will formulate a reliable sensory-motor battery which can be applied to a paediatric population with neuromotor impairment. This battery will be used to evaluate the presence of sensory impairments in children with hemiplegic CP The relationship between sensory deficits and SEP findings in these children will be explored. The correlation between the extent of sensory loss and the level of motor disability will be evaluated within subjects as well.

In Chapter 1, the literature on the somatosensory system, existing sensory tests, and sensory involvement in CP, as measured by clinical and electrophysıologic methods, is reviewed After careful survey of the literature objectives of this study are outlined. The following chapter summarizes the methodology used in my study. The selection criteria for subjects and controls, the experimental design, and the assessments used for the examination of sensory and motor function are delıneated. The thırd chapter discloses the findings of this study. The fourth chapter discusses the relationship between my study with previously performed research. Results of this study and their relevance to clinical practice are highlighted, and original contributions are emphasized

In this study a standardized sensory assessment battery for school-aged children was formulated, which can be applied to patients with neuromotor injuries. The extent and nature of sensory deficits in hemiplegic children was also illustrated The significance of these findings among health professionals, who treat children with CP is emphasized. 


\section{ACKNOWLEDGEMENTS:}

Thank you God for being there for me to lean on whenever I needed someone.

I would like to express my heartfelt gratitude to my parents for being able to let go, and giving me the opportunity to be able to pursue my degree. Also sincere thanks to all of my family members for their support.

* To my supervisor Dr. Annette Majnemer, without whose guidance and support this Master's degree would not have been accomplished.

* To Dr. Bernard Rosenblatt, many thanks for his patience in dealing with my endless questions.

* Sincere gratitude to Dr. Watters, Dr. Silver, Dr. Shevell and the Shriners Hospital for recruiting subjects.

* To Rena Birnbaum and all the staff of the Occupational Therapy Department of the Montreal Children's Hospital for their unswerving help.

* To Kathy, Jocelyn, Annick and all the EEG staff of the Montreal Children's Hospital for technical assistance.

* To Dr. Jones and Professor Dannenbaum for their advice

* To Barbara Mazer for her objective and critical appraisal of my work.

I am very grateful to the parents and children who have participated in this study.

Last but not the least, to all my friends for their support throughout the duration of this degree. 


\section{LITERATURE REVIEW}

\subsection{DEFINITION AND CLASSIFICATION OF CEREBRAL PALSY}

The first step is to have an understanding of the phenomenon called Cerebral Palsy (CP). CP is a global diagnostic category encompassing a group of disorders with multiple etiologies, neuropathologies and symptomatologies, grouped together for convenient managemient (Boone, 1978; Brown, 1984). Though emphasis has always been placed on aberrant motor control and posture which are its primary characteristics, sensory deficits are often thought to be present (Hohman et al, 1958; Tizard et al, 1954).

To date, CP remains a therapeutic challenge. Its uncertain rtiology frustrates attempts at prevention, and therapeutic efficacy had not been easily determined because of its poorly defıned prognosis (Lord, 1984).

In the U.S.A., CP occurs in every 1 to $5 / 1000$ live births it is estimated that approximately 400,000 living children in the U.S.A. have CP (Lord, 1984). It is one of the most common, chronic childhood handicapping conditions.

In 1956, Minear developed a classification for the American Academy of Cerebral Palsy based on motor symptoms corresponding to the suspected site of lesion. For example, the spastic form of CP could be due to a lesion of the cerebral motor cortex and/or a lesion of the internal capsule (Chapman and Wiesendanger, 1982) There have been some criticisms of this system of classifiration It was considered to be a very rigid form of classification for the very young infant, whose central nervous system is still developıng, and in whom there may be changes in symptomatology as the central nervous system matures (Clark and Allen, 1985).

Minear's classification can be expanded to include the severity, clinical presentation and anatomical distribution of the injury. Thus $C P$ can be of the mild, moderate or severe types. It can be clinically classified as spastic, athetoid, ataxic, flaccid or mixed (Appendix A). It may be classified according to the number of limbs involved. These clinical manifestations would include diplegia, monoplegia, hemiplegia, and quadriplegia. 
The possible etiologies and the associated neuropathology, and clinical features in static encephalopathy will now be described. Hypoxic-ischemic brain injury is 1 of the major causes of static encephalopathy (Volpe, 1987). There are 2 mechanisms by which the perinatal brain can face oxygen deprivation. These include hypoxemia, in which the oxygen in the blood supply is decreased, and ischemia, where the blood supply to the brain is diminished. Both hypoxemia and ischemia can occur as a result of asphyxia or impaired exchange of gases (i.e. oxygen and carbon dioxide). The maturity of the brain at time of the injury, and the severity and duration of the injury, will influence the nature of the hypoxicischemic lesion (Hill, 1991). Hypoxic-ischemic encephalopathy presents with 5 major patterns of brain injury. They are selective neuronal necrosis, status marmoratus, parasagittal injury, periventricular leukomalacia and focal/multifocal ischemic brain necrosis. I shall briefly review them all.

Selective neuronal necrosis is felt to be the most common type of hypoxic-ischemic injury in the neonatal brain. It often presents itself with the other varieties of hypoxic-ischemic injury. Neurons of the cerebral cortex, hippocampus, cerebellum, thalamus, reticular formation and brain stem nuclei are selectively vulnerable to brief periods of anoxia and result in neuronal necrosis. Increasing evidence is being obtained regarding the close relationship of these areas selectively vulnerable to neuronal necrosis and the anatomic distribution of glutamatergic nerve terminals (Hill, 1991). Following asphyxia the concentration of excitatory glutamate is high, which is felt to be destructive to the brain. Long-term clinical features exhibited include mental retardation, spastic motor deficits (i.e. mainly spastic quadriparesis), seizures, bulbar and pseudobulbar palsy, ataxia and attention disorders (Volpe, 1987).

Status marmoratus has been noted to be the least common of hypoxic-ischemic manifestations. It is most commonly seen in full term neonates and involves injury to the thalamus and basal ganglia following partial prolonged hypoxia. Clinically, extrapyramidal involvement is manifested by bilateral choreoathetosis, dystonia 
and resting tremor. Mental retardation of varying degrees and spastic quadriparesis may also be seen.

Parasagittal cerebral injury refers to involvement of the cortex and adjacent white matter parasagittally or in the superiomedial aspects of the cerebral convexities following ischemia (Volpe, 1987). The areas of injury are the end zones or watershed areas of the anterior, middle and posterior cerebral arteries, which are most susceptible to injury due to a diminished cerebral blood flow. In the fetal brain many anastomoses are present between the cerebral arteries. However these anastomoses regress as the fetus approaches term. Thus parasagittal cerebral injury has been most often noted in full term infants. The term 'watershed infarct' has often been used to describe parasagittal injury, which usually affects the posterior regions of the parieto-occipital lobes. This injury is usually perceived as bilateral with greater degree of involvement of 1 hemisphere. Clinical features include spastic quadriparesis (upper limbs tend to be more involved) and intellectual and perceptual disturbances (site of lesion contributing to visuomotor and auditory deficits) (Volpe, 1987).

Periventricular leukomalacia refers to the involvement of white matter adjacent to the anterior and posterior ends of the lateral ventricles, the centrum semiovale and the optic and acoustic radiations. The cerebral arterial borders and end zones (I.e. watershed regions), are the most susceptible to dimınıshed cerebral perfusion in the full term infant. The development of periventricular leukomalacia is primarily in the immature brain, as the blood supply is shunted primarily to the periventricular region which is therefore vulnerable to hypoxic-ischemic insult. Intraventricular hemorrhage has been described as a large contributing factor to periventricular leukomalacia. Clinically infants with periventricular leukomalacia exhibit spastic diplegia and mental retardation (Volpe, 1987).

Focal and multifocal ischemic necrosis include large, focal regions of necrosis, within the distribution of the 3 major cerebral arteries. Hill (1991) states the prevalence of neonates with asphyxia who exhibit this type of necrosis as $15-20 \%$. Incidence is greatest between $37-40$ weeks of gestation, with the middle 
cerebral artery being implicated in almost $50 \%$ of the cases. Cavity formation frequently occurs along with focal and multifocal ischemic necrosis. High water content along with a lesser degree of myelinated fibres and decreased astroglial response contribute to a tendency for the immature brain to undergo cavitation. Thus porencephaly (single unilateral cavity), hydranencephaly (large bilateral cavities filled with cerebrospinal fluid) and multicystic encephalomalacia (mainly bilateral multiple cavities) may ensue. Long-term neurological sequelae include hemiparesis or quadriparesis depending on the extent of the lesion. Fifty to sixty percent of cases with congenital hemiplegia have focal necrosis involving the middle cerebral artery. Porencephaly is usually associated with impaired intellect. Bilateral cerebral involvement is often thought to occur with porencephaly, with greater degree of injury to 1 hemisphere. Seizure disorders are also commonly seen in children with focal and multifocal lesions (Volpe, 1987).

Metabolic factors such as faulty glucose, bilirubin and amino acid metabolism, play an important role in static encephalopathy. Glucose is the primary metabolic fuel needed for the brain to function. Hypoglycemia makes the brain more vulnerable to ischemic injuries. Thus hypoglycemia concomittant with hypoxemia or asphyxia increases the risk and degree of brain insult, when compared to brain injury possible with hypoxemia or asphyxia alone (Volpe, 1987). The incidence of hyperbilirubinemia sufficient to cause kernicterus has been significantly decreased with the development of new therapeutic measures. However disruption of bilirubin metabolism is still present and should be monitored carefully. Bilirubin encephalopathy is usually associated with extrapyramidal features of athetosis or dystonia.

Aside from hypoxic or ischemic insults, intracranial infection, perinatal trauma and maternal exposure to teratogens could also cause static encephalopathy. Thus a variety of neuropathological factors could be responsible for giving rise to static encephalopathy. This can be manifested in a multitude of symptoms, which when grouped together for clinical convenience contribute to the phenomenon of $\mathrm{CP}$. 
In order to increase our appreciation of the range and character of sensory deficits probable in CP, and their effect on movement according to the site and extent of lesion, a background of the various components of the somatosensory system and the effect of lesions at various levels will be reviewed.

\subsection{SOMATOSENSORY SYSTEM}

This section will summarize the somatosensory system and the sensory impairments that are evident following lesions at varying levels.

Sensation is a general term that refers to the reception of sensory information (Guyton, 1991). Sensory receptors can be classified according to the nature and type of stimulation they receive and their location in or on the body. Thus, there are 5 types of receptors which include: mechanical, thermal, pain, chemical and electromagnetic. These somesthetic receptors collect sensory information from the body. For example, mechanoreceptors collect tactile (touch, pressure and vibration) and kinesthetic inputs Mechanoreceptors can be further divided into the rapioly adapting (resporise occurs at onset and removal of the stimulus), and slowly adapting (respond with sustained discharge). Factors such as temperature, force, velocity and sharpness of the stimulus will characterize the sensory experience (Martin and Jessel, 1991)

The somatosensory system consists of pathways that transmit peripheral cutaneous inputs along ascending pathways via the spinal cord, brainstem and thalamus to the cerebral cortex (Norsell,1980). The 2 important ascending central pathways are the dorsal columnmedial lemniscal system and the anterolateral spinothalamic system. The dorsal column-medial lemniscal system relays information via the ventroposteriolateral (VPL) nucleus of the thalamus to the anterior parietal lobe (Martin and Jessel, 1991). The peripheral input received by the VPL nucleus of the thalamus is also relayed to the motor cortex (Asanuma and Arissian, 1982; \& 1984).

Fibres of the spinothalamic pathway synapse on the neurons of 3 thalamic regions i.e. the VPL which projects to the primary somatosensory areas of the cortex, the intralaminar nuclei which 
project to areas of the cortex and basal ganglia, and the posterior nuclei which project to areas of the parietal lobe excluding the primary somatic cortex (Martin and Jessel, 1991).

The dorsal column-medial lemniscal system mediates tactile localization and fine discrimination, as well as phasic sensations such as vibration, kinesthetic awareness and pressure. It is also important for the performance of skills that require spatial resolution and precise timing of somatosensory feedback (Martin and Jessel, 1991). The spinothalamic system mediates pain, thermal input, protopathic touch and pressure, tickle, itch and sexual sensations (Guyton, 1991).

The primary sensory impulses are transmitted to the somesthetic cortex. This cortical region is divided into Somatic Sensory Area I (S I) and Somatic Sensory Area II (S II). The Posterior Parietal Lobule is also involved in sensory mediation.

Studies of the somatosensory cortex have been crucial in developing a better understanding of the underlying cortical mechanisms. S I includes the post-central gyrus and depths of the central sulcus (Dykes, 1978). S I had been divided into cytoarchitectonic areas 3,1 and 2 by Broadmann, and Vogts had further subdivided area 3 into $3 a$ and $3 b$ (Kaas, 1983; Semmes and Turner, 1977). Each area appears to respond preferentially to specific receptors. Thus, the majority of the neurons in $3 a$ are activated by subcutaneous muscle spindle afferents, area $3 b$ by cutaneous inputs, area 1 by a mixture of cutaneous and deep afferents, and area 2 neurons are excited by deep receptors. Area 1 can be differentiated from area $3 \mathrm{~b}$ as it has been shown to receive Pacinian corpuscle inputs (Dykes, 1982; Paul et al, 1972). Recent studies have shown the existence of many homunculi in the $S I$ of higher primates. Both area $3 \mathrm{~b}$ and 1 carry a detailed map of the body surface (Dykes, 1978; Kaas et al, 1979).

Lateral and somewhat posterior to $S$ I lies S II, which is located in the upper bank of the lateral sulcus (Kandel and Jessel, 1991). Sensory inputs to $S$ I originate from the contralateral body surface, whereas S II receives inputs from both the ipsilateral and the contralateral side of the body (Dykes, 1982; Kandel and Jessel, 
1991). Afferent inputs are also relayed to the posterior parietal lobule consisting of Broadmann area 5 and portions of area 7. Seal (1989) propounds that some selective area 5 neurons play a role in transformation of sensory activity into motor activity.

Lesions at different levels of the somatosensory system cause a variety of sensory deficits. For example, permanent impairment of pressure discrimination, loss of sensation of direction and form for stimuli moving across the skin, loss of graphesthesia, as well as decreased distal limb dexterity are seen in primates with ipsilateral dorsal column lesions. Asanuma and Arissian in 1984 studied the effect of severing the dorsal columns and $S \mid$ both individually and simultaneously. Elimination of both inputs produced severe motor deficits, whereas temporary motor impairment was documented when only one of the sensory areas was excised. Complete lemniscal lesions cause permanent deficits of graphesthesia, vibration, two-point discrimination (2pd), tactile discrimination, tactile localization, loss of direction sensitivity and proprioception. Unilateral lesions of the thalamus involving the VPL complex may cause defects on the contralateral side of the body involving the modalities of pressure, temperaiure and kınesthesia (Norsell, 1980). Removal of the $S I$ in primates may result in sensory deficits such as decreased 2pd, point localization, stereognosis, position sense, kinesthesia and tactual discrimination on the contralateral side (Carlson, 1981; Corkın et al, 1970; Darian-Smith et al, 1982; Roland, 1987; Semmes and Turner, 1977)

Severe impairments in tactile learning and memory may result from bilateral excision of $S \mid l$ with relative sparing of tactile sensitivity, or weight discrimination (Dykes, 1982; Ridley and Ettlinger, 1976; Semmes and Porter, 1972). Thus S II probably has a greater bearing on sensoiy-motor integration than on discriminatory uses (Roland, 1987).

Posterior parietal lobule lesions or excision leads to complex motor and sensory deficits (Hyvarian and Poranen, 1974; Seal, 1989). Spatial disorientation and stereognosis of the contralateral body surface (Roland, 1987) and severe disturbance in manipulation of objects (Deuel and Regan, 1985; Pause and Freund, 1989) are seen 
with posterior parietal cortex lesions. Human studies have also shown that unilateral lesions of the cerebral hemisphere result in bilateral sensory deficits (Carmon, 1971; Corkin et al, 1973).

While considering evaluation and treatmerit of a central nervous system injured patient, one must keep in mind the plasticity of the brain (Bach-y-Rita, 1981; Kaplan, 1988; Moore, 1986). Factors responsible for long term recovery are mainly collateral sprouting and unmasking of functionally depressed pathways (Bach-y-Rita, 1981). This may cause changes in the mapping and thus affect the recovery of a patient with a central nervous system injury.

Effective evaluation and rehabilitation of patients with central nervous system lesions depend on our understanding of the underlying neurophysiologic and pathologic features (Twitchell, 1958). Several studies have described the impact that sensory deficits may have on hand function (Bolanos et al, 1989; Curry and Exner, 1988; Green and Banks, 1962; Van Buskirk and Webster, 1955; Westling and Johansson, 1984). Curry and Exner (1988) state that sensory experience (like tactile and proprioceptive cues), is based on active movement. Thus, a child with motor limitations will have less sensory input. The authors observed that it is not movement that is learnt, but the 'sensations of movement'. Therefore, a lack of sensory information perceived may lead to impairment of hand function.

The prehensile ability of the hand is one of its most important and unique features. For example, a precision grip is used to manipulate small objects being held. There is a close relationship between sensory feedback, and the explorative movements of the fingers over the environment, and the corresponding fine adjustment of grip force during performance of tasks of daily living (Dannenbaum and Dykes, 1988). Studies have repoited that lesions of the pyramidal tract and dorsal columns in primates lead to severe impairment of precision grip (Westling and Johansson, 1984). The hand is used less functionally when severe sensory deficits are present even in the presence of good motor control (Curry and Exner, 1988; Green and Banks, 1962; Van Buskirk and Webster, 1955). In their study of $96 \mathrm{CP}$ children, Tachdjian and Minear (1958) found that in the presence of a 
sensory deficit hand function was no better than fair. Thus hand function can be significantly limited because of poor sensory function. Bolanos et al (1989) recommended that sensory testing should be an integral part of therapy, as tactile sensations/stereognosis are closely linked to the performance of tasks requiring finger dexterity and tactile gnosis. Therefore, sensory testing is critically impoltant when considering rehabilitation options to improve hand function in subjects with central nervous system dysfunction.

The next section deals with the methods used to evaluate the various sensory modalities, as well as the clinical and electrophysiologic tools currently available to assess sensory function.

\subsection{SENSORY TESTS}

Many studies in the literature report on the debilitatıng effects sensory deficits may have on motor function in patients with peripheral nerve lesions. Not only does this have a profound influence on the independence and vocation of the individual concerned, but may also compromise his/her family (Dellon, 1983) At present, there is a need for better identification and characterization of sensory deficits in central nervous system disorders in paediatric age groups (Curry and Exner, 1988).

Accurate sensibility testing aids physicians and therapists in identifying hand pathology and dysfunction, and in evaluating the efficacy of ongoing treatment. Many tests that measure cutaneous sensibility have been developed. However, a simple yet thorough, objective and reliable test is still needed, particularly for the paediatric population (Bell, 1984).

\subsubsection{Sensibility Tests}

Sensibility is defined as the ability to feel or perceive (Callahan, 1984). There are various sensibilities which can be tested using different methods. Pressure sensitivity can be tested with nongraded or graded instruments. The reliability of the testing is improved by using an instrument that can grade the amount of 
pressure applied to the skin from light to deep. Currently the most widely used measuring instrument is the Semmes-Weinstein Aesthesiometer (Bell, 1984). This is a variation of the amount of hairs developed by Von Frey in 1895. The Semmes-Weinstein Aesthesiometer was developed to Identify somatosensory changes in brain-injured adults (Semmes et al, 1960). The kit consists of 20 probes, each probe having a nylon monofilament attached to a Lucite rod. They are numbered from 1.65 to 6.65 according to the logarithm of 10 tımes the force required to buckle the monofilament. It had been shown to have a very high test-retest reliability and its force of application can be controlled. However, slippage of the filament on application and improper storage can decrease its reliability (Callahan, 1984; Jones, 1989; Levin, 1978).

Two-point discrimination ( $2 p d)$ is the ability to distinguish between 2 non-noxious light-touch stimuli which are applied simultaneously to the skin (Nolan, 1983). It can be tested with paper clips, or calipers with blunt ends such as the Enley gauge. However the reliability of these instruments is questionable because of the variability in the amount of force applied and the angle of application of the force (Callahan, 1984; Jones, 1989). The 2 point aesthesiometer consisting of 2 parallel filaments has been suggested as being the most reliable instrument for measuring $2 \mathrm{pd}$. However, the amount of pressure used is arbitrarily selected. Furthermore, a precise measurement of the distance between the 2 tips of the aesthesiometer is required to establish reliability. The width of the tips may vary and thus cause a poor inter-rater reliability (Jones, 1989). Dellon et al (1987) showed that moving 2pd measured with a Disk-criminator has a very high reliability.

Static two-point discrimination ( $22 p d$ ) is mediated by the slowly adaptıng receptor system and is an important factor in precision grip (Dellon, 1984). Moving two-point discrimination (m2pd) on the other hand is mediated by the rapidly adapting receptor system and is an important component of object manipulation and hand function. M2pd was found to be a better predictor of hand function when compared to s2pd in subjects with peripheral nerve dysfunction (Dellon and Kallman, 1983). 
Somesthetic information about the environment is primarily obtained by primates by the movement of their hands over an object (Essick and Whitsel, 1985). Mechanoreceptors detect the objects shape and texture, as well as the speed and direction of the object's movement across the skın. This input is mainly transmitted by the dorsal column-medial lemniscal system (Essick and Whitsel, 1985) Direction sensitivity has been shown to be an important component of graphesthesia and sterengnosis. Thus, evaluation of direction sensitivity should facilitate study of cutaneous dysfunction (Essick and Whitsel, 1985). To my knowledge none of the studies in the literature have tested directionality, but it would be an important test to assess.

Proprioception is the position of different parts of the body in relation to each other and in space. Excision of $S I$ in primates has been shown to result in a loss of proprioception (Corkın et al, 1970 , Darian-Smith et al, 1982; Roland, 1987). Thus, CP could result in an impairment of proprioception. Studies done by Hohman et al (1958), Tachdjian and Minear (1958) and Tizard et a! (1954) showed that proprioception was often affected in children with $C P$ Therefore, this would be an important somesthetic sense to evaluate Many studies have evaluated position sense in CP However the method of testing proprioception is often not specified. Proprioception was usually tested at the shoulder, elbow and joints of the hand, by asking the subject to identify the direction in which the joint was moved with vision occluded (Hohman et al, 1958; Jones, 1976 , Kenney, 1963, Tachdjian and Minear, 1958; Tizard et al, 1954, Twitchell, 1958).

Stereognosis is the perception and identification of the form and nature of objects by palpatinn with vision occluded (WaylettRendall, 1988). It can be measured by various tests. Dellon's modification of the Moberg pick-up test (tımed object recognition test) are often used. Here, 1? fixed objects are placed in a box Within a given time frame the subject is asked to identify each object placed in his hand, with vision occluded. This test relies on intact motor function (Callahan, 1984). 


\subsubsection{Adult Sensory Tests}

The functional level of the hand is dependent on its sensory ability. Therefore a valid and reliable tool is required for the effective management of patients with brain damage.

Although many methods for evaluation of motor function have been validated with high inter-rater as well as test re-test reliability, sensory assessment batteries for central nervous system disorders remain controversial and are in need of standardization (Dannenbaum and Dykes, 1988).

Currently, tests which are used to assess sensory function following central nervous system lesions have been adapted from tests designed to evaluate sensory loss of a peripheral nerve injured population (Dannenbaum and Dykes, 1988). For example, the tests of vibration (256 and 30 cycles per second), $\mathrm{m} 2 \mathrm{pd}$ and s2pd were combined by Dellon to assess sensory function of the hand after nerve repair (Dellon, 1984). Gelberman et al (1983) examined s2pd, m2pd (caliper), pressure sensitivity (using Semmes-Weinstein monofilaments), and vibration (tuning fork at 256 cycles per second) in 12 adults (19-41 years), with experimentally simulated median nerve compression. The authors found that the monofilaments and vibration were more sensitive measures of detecting decrease in sensory function.

\subsubsection{Paediatric Sensory Tests}

Paediatrıc test batteries, as compared to adult assessments, should have minımum expressive and receptive language requirements. The testing environment and the materials utilized should be modified to suit a paediatric population. The sensory tests which have been standardized for a paediatric population are generally not suited for neurologically handicapped children due to a greater degree of receptive (understanding complicated instructions) and expressive (complex answers required) language skills and motor abilities (e.g. graphesthesia needs sophisticated finger mobility), as well as good attention span required to complete the sub-tests (Hacker and Porter, 1987). Furthermore most of the existing paediatric assessments are not standardized on a school- 
age population. Thus, these assessments may not reliably assess sensory function in CP children of school age.

Few standardized paediatric test batteries that assess sensory function are available. Sensory components of the Quick Neurological Screening Test (QNST) (Mutti et al, 1978), Miller Assessment for preschoolers (MAP) (Miller, 1982), and Sensory Integration Test (SCSIT) (Ayres, 1980) are briefly described. These are the only standardized tests available which include aspects of tactile sensory testing appropriate for a paediatric population.

- The QNST consists of 15 observed tasks administered to children who are suspected of being learning disabled. The sensory items include graphesthesia, and double simultaneous stımulation The examiner needs to be well practised in admınıstering the QNST Graphesthesia is assessed by asking the child to identify 4 numbers drawn on each palm. Graphesthesia evaluates the sensory, as well as, perceptual capacity of the child. The numbers are traced on the palm by the examiner, with a fingertip or the blunt end of a pencil Thus the amount of pressure being placed on the palm is variable Administration of double simultaneous stimulation of the hand and cheek requires precise coordination on the part of the examiner. The test examiner must also be skilled in making qualitative observations required in this assessment, therefore scoring in this battery is subjective. It has been standardized on healthy and learning disabled children under 6 years to over 17 years However the 6-12 years cohort of learning disabled children is very small Test-retest reliability is good $(r=081)$, and inter-observer reliability is fair $(r=0.71)$.

-The MAP is a sensory-motor evaluation that is used clinically for preschool children (2 $1 / 2$ - $51 / 2$ years) with mild developmental deficits. It tests stereognosis, finger localization and kınesthesia Stereognosis was evaluated by asking the child to identify a penny, safety pin, rubber band, eight point star, key, moon, circle, trapezoid a scallop shape and a triangle, by tactile matching. For a test to be used with children, the objects used for stereognosis should be appropriate for a paediatric population. Therefore the need for a modified stereognosis test which is more geared towards children is 
apparent - it should be able to hold their interest, the objects used should be easily recognizable and be small enough to fit in their hand. Finger localization is tested on the proximal phalanx of 2 fingers of the hand. This test is difficult to administer in a standardized manner (Miller, 1982). The distal phalanx as compared to the proximal phalanx is a more functional area to test, because the environment is explored with it. Kinesthesia is tested by asking the child to imitate a posture (Simon says game). This requires a combination of sensory, motor and cognitive abilities (Stowers and Huber, 1987). Although the MAP has been standardized in preschoolers, these subtest items may not be good at reliably evaluating sensory function in this age group, as neurophysiological and psychological maturation is not complete. The MAP consists of a well organized test kit and has normative data based on the scores of 1200 healthy children. However it is not readily useable for a physically disabled population (CP).

-The SCSIT has been designed for school-age children (4.8 - 8.9 years) with learning difficulties. It tests kinesthesia, manual form perception, finger identification, graphesthesia, tactile localization and double tactile stimulation. Kinesthesia of the shoulder and elbow are tested by asking the child to place a finger on a spot previously toucned by the tester with vision occluded. Graphesthesia is tested by asking the child to identify the line drawing done on the back of their hand, and then to duplicate it with the other hand on the same place. Tactile localization is assessed by asking the child to localize touch on hands and forearms with vision occluded. These tests assess the sensory ability of the arm, forearm and dorsum of the hand. However, emphasis should be placed on the volar surface of tire hand since fine skillful activity is mainly a function of the hand. Furthermore, these are not very objective and reliable measures of sensory function [e.g. tactile localization could be more reliably assessed using a filament (Jones, 1989)]. The sensory tests performed in the SCSIT not only evaluate sensory function, but also require perceptual skills. The SCSIT has been criticized by many authors (DeGangi, 1987) for inadequate standardization procedures used (particularly regarding interpretation of standard scores and 
poorly described demographic information), poor reliability on most of the tests (only test-retest reliability has been reported with no information on the time difference between 2 assessments performed) and inadequate information regarding validity studies performed. Overall, the SCSIT is considered inappropriate to evaluate children with motor disabilities.

Thus, though all these tests do cover some aspects of sensory testing, none of these tests have been designed for clinical use in a paediatric population with significant central nervous system involvement and specifically with neuromotor impairment. Objective instruments (i.e. filaments, Disk-criminator), and standardized methods that yield more accurate sensory input are not always used. Therefore, accuracy of the results of these assessments could be confounded by the subjective nature of the testing and scoring.

\subsubsection{Somatosensory Evoked Potentials (SEP)}

An evoked potential (EP) is an electrical manifestation of the brain's reception of and response to an external stimulus (Chiappa, 1990). By stimulating a special sense organ or sensory nerve fibres such as the median nerve (a mixed nerve containing both sensory and motor fibres), a response is collected from the surface of the scalp using surface electrodes. An SEP can be generated by physiologic or electrical stimuli, however the latter is preferred because it is more easily controlled and yields more reliable responses. The action potential volley passes through the brachial plexus dorsal root entry zone, posterior columns, nucleus cuneatus, medial lemniscus, thalamus, thalamocortical radiations and cortex respectively. Electrodes are placed at the Erb's point (EP), over the second cervical vertebra (C II), and along the skull over the contralateral parietal lobe (Cc) using bony landmarks and the 10-20 International System of electrode placement. A series of waveforms are generated that reflect functional activity along the ascending pathway. The direction of trace movement is dependent on the way the amplitude and display unit electronics have been programmed to respond to a potential difference between Grid 1 and Grid 2 (the 2 input electrodes). EEG convention states that Grid 1 is negative with 
respect to Grid 2, thus yielding an upward deflection (Chiappa, 1990). There is no universally recognized convention in evoked potential studies. Three methods exist for SEP nomenclature. Components can be numbered in sequence by polarity; for example $N 1, N 2, N 3$ etc ( $N=$ negative). Waveforms can also be labelled according to their polarity and mean latency in healthy subjects; for example N20, P100 etc. Lastly the name of the generator site of the waveform could be used; for example EP - erb's point (Chiappa, 1990).

The utility of the SEP stems from the close relationship between the evoked potential waveforms and specific structures (Chiappa, 1990). Changes in SEP such as an increase in central conduction time (latency difference between 2 central peaks) or the absence of peaks may reflect central nervous system dysfunction due to a lesion or a pathologic process such as demyelination (Wong et al, 1982).

SEP are a non-noxious, non-invasive method of evaluating the integrity of the dorsal column-lemniscal pathways. However its utility and reliability may depend upon a variety of factors such as available norms, effects of developmental changes due to maturity of the somatosensory system, varying height, ease of measurement and effect of sleep. Furthermore, controversy exists surrounding the interpretation of the SEP waveform. However, they are well tolerated by children and the waveforms obtained are easily reproduceable due to minimal artifact present (Fagan et al, 1987, Laureau et al, 1988).

Useful information regarding the maturation and functional integrity of the nervous system can be obtained through SEP findings in the paediatric population. SEP are a valuable tool in the evaluation of children with both peripheral and central neurological impairments (Cracco, 1989).

Thus it would be of interest to study the extent of sensory dysfunction in CP children using a clinical sensory assessment as well as SEP. To facilitate this process, the next section will briefly review studies which have assessed sensory function in static encephalopathy using both clinical and electrophysiologic procedures. 


\subsection{SENSORY TESTING IN STATIC ENCEPHALOPATHY}

In the U.S.A. $0.1-0.5 \%$ of children have Cerebral Palsy (C.P). Thus $\mathrm{CP}$ is a chronic, highly prevalent childhood handicapping condition (Lord, 1984). To date CP remains a therapeutic challenge. Though motor deficits have been emphasized, sensory dysfunction may exist and may contribute to diminished use of the affected hand (Hohman et al,1958; Tizard et al,1954).

\subsubsection{Clinical Sensory Testing In Cerebral Palsy}

The clinical description of CP emphasizes motor dysfunction, obscuring other important symptoms (Boone,1978; Curry and Exner,1988). Studies evaluating the performance of CP children reveal the existence of sensory deficits of various types in $40-73 \%$ of the patient population (Hohman et al,1958; Jones and Ogg,1966; Kenney,1963; Tachdjian and Minear,1958; Tizard et al,1954). Form and tactual discrimination, light touch, temperature, two-point discrimination (2pd), point localization and weight perception have been evaluated. In all of these studies, stereognosis, $2 \mathrm{pd}$ and position sense were the sensory modalities chiefly impaired.

Tizard et al (1954) studied the sensory status of 106 children with congenital and acquired hemiplegia. Fifty percent of the children were found to have sensory deficits. Interestingly, they found that sensory impairment did not depend on the age at which the injury occurred. Children with both congenital and acquired hemiplegia had deficits in stereognosis and $2 \mathrm{pd}$. In addition, the children with acquired hemiplegia also had deficits in their discrimination of joint position, touch, pain and temperature The sensations were tested in the conventional manner, however, the measurement techniques and materials used were only briefly outlined. Two-point discrimination was measured by the two ends of a paper clip. This method has been found to be unreliable due to variation in both the width of the ends of the paper clip and in the adjustment of the distance between the two ends (Jones,1989). No mention of a control group is made and one is left to presume that 
the unaffected side was used as a control. Also, how cut-offs were made to determine sensory impairment was not delineated.

Hohman et al (1958) examined $47 \mathrm{CP}$ children ranging in age from 6 to 16 years. All children had either hemiplegia or spastic quadriplegia with more involvement of one side of the body. Thirteen sensory tests were performed and 34 of the children (72\%) were found to have sensory involvement. The most common modalities impaired were stereognosis, 2pd, and position sense. Interestingly, in some cases, small areas of the hand were affected with the rest of the hand having good sensation. Unfortunately, not all sensory tests were done reliably. The site of testing was mentioned in very general terms. Hot and cold differentiation was done using metal nutpicks. With the use of hot and cold differentiation one has to ascertain whether the subject is actually differentiating cold from hot, or rather cold from not cold (Callahan,1984). 2pd was measured by a divider which could be unreliable due to slight variation in adjusting the distance between the two ends (Jones, 1989). Furthermore the pointed ends of the divider may be assessing pain perception rather than touch (Bolanos et al,1989). Scoring criteria were not clarified and the cut-offs used for scoring sensory impairment were not described. Thus, in spite of unreliable methods of sensory assessment and errors in statistical evaluation, this study demonstrates that children with $C P$ have sensory deficits.

Monfraix et al (1961) stated that examination of various agnosias such as tactile gnosis, and body image are an integral part of evaluating a CP child's function. In a preliminary study, the authors have established stereognosis norms on 218 controls evaluating their ability to recognize 5 objects and 12 shapes. The degree of tactile gnosis could be measured depending on the gnosic age as compared to the mental age of the child. The authors then studied the tactile gnosic ability of $92 \mathrm{CP}$ children (Monfraix and Tardieu, 1961). Fourteen of the 22 hemiplegic children had tactile agnosia. Five of these hemiplegic children had bilateral agnosia showing that the non-affected side of hemiplegics is not functioning normally. Twenty-seven of the 52 children with bilateral motor disorders demonstrated tactile agnosia as well. The investigators state that 
re-education of tactile gnosis could be done, however success would depend on whether the tactile gnosis was caused by a cortical lesion or was due to disuse of the hand (Monfraix and Tardieu, 1961).

Curry and Exner (1988) used 5 pairs of objects with different textures, to determine the nature of textures consistently preferred by 15 preschool controls and $15 \mathrm{CP}$ children. They found that CP children chose hard objects significantly more often than they chose soft objects, which may be due to decreased tactile sensitivity and the need for greater magnitude of proprioceptive cues.

Lesny (1971) recognized that sensory input is necessary for any complex motor action. He measured 2-point discrimination (2pd) thresholds with Weber's scissors, and compared results in $143 \mathrm{CP}$ children to scores in 30 controis (6-15 years of age). It was found that the CP children, especially those with hemiplegia and diplegia, had significantly increased $2 p d$ thresholds. The most significant deficit was on the distal part of the third finger.

Brown et al (1987) evaluated 25 hemiplegic children, aged 5-15 years. They conducted clinical neurological tests (range of motion, muscle tone and power, speed of movement, fatiguability and sensory function), tests of hand function and neurophysiological tests (muscle power, speed of movement, fatiguability and tremor). Position sense, tactile localization, weight discrimination, graphesthesia, finger agnosia and pain were the sensations measured. Particulars regarding the method of assessment, areas assessed or the scoring procedures were not elaborated on. They found that these tests did not differentiate between the 'normal' and affected hands. However, they found a large developmental variation in subject scores. As a result of these findings, the authors concluded that there was a need for more detailed assessment with the comparison of sensory function in hemiplegic children to that of an age-matched control group.

Several studies have claimed that children with athetosis have fewer sensory deficits than children with spastic CP (Kenney,1963; Monfraix et al,1961; Tachdjian and Minear,1958). This may be due to differing lesion sites (Kenney 1963). In the study by Monfraix et al (1961), only tactile gnosis was tested in 92 CP children. Tactile 
agnosia was found to exist in all forms of $C P$, with the most significant deficits occurring in spastic CP. Kenney (1963) tested 19 children with CP; 6 had athetosis and 13 were spastic. The authors did not disclose whether the disabilities were unilateral or bilateral. He compared their performance on various sensory tests to 27 controls (aged 5-14 years). Kenney reported that the athetoid CP children showed sensory defects only in size differentiation, whereas the spastic children had multiple deficits. In this study there were many methodological weaknesses. The objects used to test stereognosis could be identified using other sensory cues such as temperature and texture e.g. a metal key as compared to a rubber ball, and cotton as compared to a wooden cube. Position sense appeared to be randomly measured either on a finger or toe without specifying whether there were differences between the two sites and the number of test trials. Temperature was tested using cold and hot water which is often an unreliable test (Callahan,1984).

Opila-Lehman et al (1985) showed that spastic CP children had a greater impairment in kinesthetic recall, when compared to athetoid CP children. It was suggested that spastic children normally have a decrease in movement experiences and therefore diminished kinesthetic input. This may contribute to the number of errors in kinesthetic recall.

Conversely Jones (1976) found that athetoid children had poor kinesthetic awareness and suggested that this may be due to the continuous barrage of random proprioceptive input that an athetoid child receives.

Tachdjian and Minear (1958) performed 13 sensibility tests in 96 CP children, 88 of whom had spastic hemiplegia. Not only were sensory deficits present in $41.7 \%$ of the children tested, but a high correlation was found between the functional ability of the hand and the severity of sensory deficits. Therefore moderate to severe motor disability correlated with maximal sensory dysfunction. The authors found that those children with moderate to severe sensory disability did not improve even after orthopaedic surgery to decrease motor disability. In this study, the contralateral side of the body was used as a control. However in many children the contralateral side may 


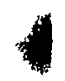


The likelihood of CP children having motor impairment due to insufficient sensory experience is significant (Curry and Exner,1988). The effect of limited sensory input on movement in babies with unilateral cerebral lesions has been studied. It was observed that babies tended to ignore the affected body part due to decreased sensory experience and failed to integrate the involved extremity into their body scheme. Hence a 1 year old hemiplegic infant would use his involved extremity far less than one would expect given the extent of physical involvement (Curry and Exner,1988). This study emphasizes the correlation between sensory input and motor function. Hence a child with a significant sensory deficit would likely have an impairment in hand function.

In summary few studies have examined the sensory function in CP children and methodological problems undermine the strength of these studies. The majority of them were done in the 1950's and 1960's. Traditional, subjective methods of test administration were used. These tests have not been standardized on children, have not been proven to be reliable, and have no normative data. Statistical procedures used in these studies were also inadequate. In spite of these methodological problems, these studies clearly indicate that health professionals working with CP children should consider that sensory deficits may be present, and that they may influence motor performance. Limitations in functional skills may reflect, in part, the type and magnitude of sensory loss (Curry and Exner,1988). In a clinical setting, effective management of a patient with CP should include evaluation of sensory deficits, with subsequent remediation (sensory retraining, for example) to maximize sensory awareness and functional performance.

Hence, it would be of interest to assess sensory function in one sub-group of $C P$, such as in hemiplegic children. I propose to assess sensory function, using a standardized paediatric sensory battery on both the so-called 'affected' and 'non-affected' side. In addition, it would be useful to evaluate the integrity of the somatosensory system not only by clinical tests, but also with a reliable neurophysiological tool. 


\subsubsection{Neurophysiological Testing (SEP) in Static Encephalopathy}

Not only are Somatosensory Evoked Potentials (SEP) a useful diagnostic tool in adult neurology, they have been shown to yield valuable information in the paediatric population (Cracco, 1989). In infants and children, SEP reliably evaluate the maturation of afferent pathways in the central and peripheral nervous system (Cracco,1989; Zhu et al, 1987). However, the clinical utility of SEP is dependent on careful interpretation of the waveforms because of the complexity of maturational changes of the central nervous system as well as changes due to increase in body stature (Bartel et al,1987; Gilmore,1989; Taylor and Fagan,1988; Zhu et al,1987).

Studies have shown that short latency SEP strongly correlate with severity of neurological impairment, magnitude of sensory deficits and leve! of functional disability in an adult stroke population (Karnaze et al,1987; Larson et al,1966; Liberson,1966; Maugiere et al, 1983; Zeman and Yiannikas, 1989).

Karnaze et al (1987) studied 49 hemiplegic subjects, (mean age. 54.6 years), who had suffered cerebral ischemia using SEP. Results obtained were compared to those of 48 healthy adults All subjects underwent neurological examination. Degree of neurological impairment was based on degree of weakness and sensory loss noted Sensations of pain, temperature, position sense, vibration and touch were evaluated. SEP abnormalities correlated with sensory deficits as well as severity of neurological impairment. However several methodological problems existed in this study. Time between onset of stroke and SEP evaluation was not specified. Controls were divided into two groups, a group of 36 controls with mean age of 31 years and the other of 12 healthy adults with mean age of 66 years, without justification. The methodology for the evaluation of sensation was not specified. The parietal SEP component is not linked to pain and temperature (Maugiere et al, 1983), and therefore should not have been included in the sensations evaluated.

Stroke may cause neurological impairment with a resultant functional loss or disability. Zeman and Yianrikas (1989) assessed functional outcome using the Barthel Index as well as length of stay, 
and evaluated the integrity of sensory pathways using SEP and sensory assessments on 39 acute stroke patients (mean age: 56.4 years) and 26 controls (mean age: 50.65 years). Specific information regarding the evaluation of light touch, stereognosis and pinprick was not provided. SEP waveforms and functional outcome were significantly correlated. Thus normal SEP correlated significantly with good functional outcome as measured by the Barthel Index and length of hospitalization. Abnormal SEP correlated significantly with a lower and more variable Barthel Index and longer length of hospitalization. Statistical correlation was also documented between SEP results and sensory evaluation. This study shows the prognostic value of sensory impairment, as measured by SEP and clinical evaluation, on the functional ability of a hemiplegic adult post stroke. When both sensory deficits and SEP abnormalities were combined, there was a greater correlation to poor functional outcome, than either factor individually.

Cerebral lesions may cause changes in the cortical component of SEP (N20). This is dependent on many factors: the structures involved (location of lesion), the extent and type of lesion, and the time interval between onset of injury and SEP recording (Wong et al, 1982). Several authors have studied the relationship between SEP abnormalities as well as functional outcome. Liberson (1966) studied SEP waveforms in 15 males with aphasia (all right hemıplegics). The SEP was flat or had a markedly lowered amplitude in 10/15 aphasics. The degree of involvement correlated well with the severity of aphasia. Deficits in somatic sensation were not described In 1976, Laget et al examined 21 patients with parietal lobe lesions They found a good correlation between clinical neurological findings (motor disability, mental retardation, seizures, astereognosis etc.) and SEP abnormalities.

Maugiere et al (1983) studied the relationship between motor and sensory deficits to specific SEP abnormalities in 22 subjects. Eight sensory modalities and 2 apraxias were assessed. Insufficient methodological details regarding testing and scoring procedures were given. The authors found parietal SEP components to be closely associated to pure astereognosis and were not linked to pain and 
temperature sensations due to different pathways of transmission. Thus SEP were thought to be of great diagnostic use.

However, despite several reports of a positive correlation between SEP and clinical sensory findings, each study described a few cases where this did not apply. Giblın (1964) found a correlation in sensory deficits and SEP alteration in $34 / 42$ patients with unilateral cerebral lesions. However, he noted normal SEP recordings in 7 patients with moderate to severe sensory defıcits (2pd, joint position sense and stereognosis). Furthermore, 1 patient with no sensory deficit had abnormal SEP. Testing procedures used for detecting sensory deficits and for grading degrees of impairment were not described.

Correlations between clinical sensory findings and SEP recordings was reported by Williamson et al (1970). Twenty cooperative subjects with unilateral lesions at or above the thalamic level were assessed. Age of subjects was not specified. Fifteen of the 20 subjects were found to have clinical sensory loss of varying degrees The authors did not specify which sensations were measured or what their criteria was for sensory loss. SEP were performed on all the subjects. Recording parameters were well described, however for each patient, 3 trials of only 64 responses were done. This number may be too low to be sure that the SEP waveform is reproduceable Sensory deficit was determined by the degree of impaired joint position sense (since that was the modality chiefly impaired in subjects). SEP abnormalities were found to correlate well with moderate to severe imparrment of joint position sense (sensory deficit) in 15 patients. Thus good correlation of sensory loss with SEP abnormality was stated as a basis for future clinical use of SEP in determining sensory loss. However, 2 patients with mildmoderate impairment of position sense had normal SEP. These false negative assessments could result in misidentifying some patients who are minimally affected.

Larson et al (1966) reported a correlation between SEP abnormalities and clinical sensory deficits in hemiplegics in the acute phase, but not during the recovery period. Wong et al (1982) found that SEP generally correlate well with sensory loss, although 
there are exceptions. In hemiplegic patients with mild involvement (the most common group), SEP recordings did not always reflect general neurological deficit and therefore SEP could not be used as an independent means of diagnosis of sensory dysfunction. Zeman and Yiannikas (1989) found a significant correlation between SEP and functional outcome in their study. They concluded that SEP correlated with sensory deficits, however only $68 \%$ of subjects with SEP abnormalities had sensory loss, and $81 \%$ of subjects with normal SEP had abnormal sensation. Thus, even though SEP generally correlate well with sensory function, exceptions exist. Hence if SEP were used as the sole means of determining sensory function, reliable identification of children with sensory deficits would not be possible. Hence clinical sensory testing is of great importance in accurately determining the nature and extent of sensory impairment.

Review of the literature suggests that sensory abnormalities exist in children with $C P$, yet delineation of the type and extent of sensory loss is needed. Reports on SEP by Laget et al (1976), Williamson et al (1970), Wong et al (1982) and Zeman and Yiannikas (1989) allude to the fact that SEP abnormalities correlate to different degrees of sensory and riotor dysfunction in subjects with static encephalopathy. However important details regarding the methodology used to clinically evaluate sensation are often not reported. Thus there is a need to further examine the relationship between SEP and specific sensory modalities.

Wong et al (1982) retrospectively studied 35 CP patients. Twelve of the 35 children were found to have sensory deficits. A new objective method using signal to noise ratio to study SEP objectively was used. The degree of variability of SEP on the nonaffected and affected sides was assessed. Thirty out of 35 SEP had greater variability. (as determined by component analysis of measurements of peak latencies and amplitudes of N20, P100, N140 and P220) on the affected side. Predictive accuracy was greatest in severe cases and least in children with minimal involvement. However, for this study long latency SEP were analyzed which are not as clinically useful as short latency SEP, due to their greater 
inter- and intra-subject variability (Cracco et al, 1979), and are more affected by state of consciousness (Laureau et al, 1988).

In summary, many studies have shown SEP to be strongly correlated with sensory deficits (Giblin, 1964; Karnaze et al, 1987; Larson et al, 1966; Liberson, 1966; Maugiere et al, 1983; Williamson et al, 1970; Wong et al, 1982; Zeman and Yiannikas, 1989) However several of these studies have inadequate descriptions of the methodology used and statistical analysis are not always rigidly applied. Several studies have noted several exceptions in which SEP findings do not correlate with sensory function (Giblin, 1964; Larson et al, 1966; Williamson et al, 1970; Wong et al, 1982; Zeman and Yiannikas, 1989). Therefore evaluation of sensory function using SEP alone could be misleading when attempting to identify sensory deficits in $C P$ children.

Majnemer et al (1987) evaluated the prognostic utility of SEP in a group of 34 high-risk newborns. Abnormal SEP (10/34) performed in the neonatal period predicted motor disturbances at 1 year of age (e.g. children with persistently flat SEP bilaterally developed spastic quadriplegia, and neonates with increased latencies had developmental delays). Therefore an abnormality in a sensory test (SEP) correlated with later motor dysfunction. This suggests that both motor and sensory dysfunction may co-exist in children with static encephalopathy. Majnemer et al (1988) also evaluated brain stem auditory evoked potentials (BAEP) in a second cohort of 34 high-risk neonates. BAEP abnormalities predicted motor outcome. However, those children with normal BAEP did not always have normal outcome. This may relate to the site of BAEP generation, which is at the level of the brain stem. Therefore lesions rostral to the brainstem may be missed. SEP were found to be a more sensitive prognostic tool of functional outcome.

These results were further supported by findings reported by Logan et al (1991). Eighteen CP children with motor deficits were studied. SEP and BAEP were performed on all 18 subjects. Visual evoked potentials (VEP) were also performed on 17 children. Fourteen out of 18 children showed SEP abnormalities. Of these, 11 had severe motor deficits and 3 had moderate motor dysfunction. 
Five out of 18 had an abnormal BAEP, and $4 / 5$ had severe motor deficits. Eight of 17 subjects had abnormal VEP (7/8 with severe motor deficit). The BAEP and VEP probably detected injuries which were multifocal in their origin and thus had severe motor deficits. In conclusion the SEP, BAEP and VEP correlated well with motor deficits, however the SEP were found to be the most sensitive tool in detecting moderate-severe motor abnormalities.

Thus SEP have been found to correlate well to clinical sensory function (Karnaze et al,1987. Maugiere et al, 1983; Zeman et Yiannikas, 1989). However, Inethod of sensory assessment was poorly described in many of these studies, and several false positive and false negative findings have also been reported. Thus, SEP alone are not a sensitive tool in determining sensory dysfunction especially in those children with mild sensory impairment. However they would lend useful information to that obtained by a clinical sensory ovaluation. SEP have also been found to correlate well to motor function in high risk newborns and CP children (Logan et al, 1991; Majnemer et al, 1987). Thus it would be of interest to further study the relationship between SEP recordings, and the level of sensory and motor disability in CP children. 


\subsection{SUMMARY OF LITERATURE REVIEW}

$C P$ is a term that is used to broadly define a multitude of disorders which have been classified according to the anatomical distribution of the lesion and the clinical symptoms exhibited. Clinical symptomatology of CP consists of prominent motor impairments and possible underlying sensory deficits (Boone, 1978; Curry and Exner, 1988). Hand function may be impaired in spite of good motor function, if important sensory deficits exist (Bolanos et al, 1989; Curry and Exner, 1988; Green and Banks, 1962; Van Burskirk and Webster, 1955; Westling and Johansson, 1984) Therefore sensory dysfunction may be linked to poor functional outcome. Thus sensation is an important part of the overall assessment of a child with central nervous system dysfunction.

Sensory tests adapted for a paediatric population need special considerations. The test should be of a short duration and be able to hold a child's interest. The state of the art of reliable standardized sensory tests for a peripheral nerve injured population has been well documented. However, there is a need for standardized sensory assessment batteries for children with neuromotor dysfunction due to a central lesion (Dannenbaum and Dykes, 1988). Currently, some developmental assessments include some aspects of sensory evaluation, however they have either been designed for a preschool population or the methods of administration of sensory input are often inprecise and therefore not reliable. Thus, there is a need to develop a standardized, reliable clinical sensory battery for a school-age paediatric population with neuromotor imparrment. SEP are a valuable non-invasive elctrophysiologic tool for measuring sensory function in children, transmitted via the dorsal columnmedial lemniscal pathways in children (Fagan et al, 1987). It would be interesting to correlate sensory function as measured by a reliable clinical battery with SEP.

Studies reported in the literature have concluded that sensory deficits are present in CP children (Hohman et al, 1958; Jones and Ogg, 1968; Kenney, 1963; Tachdjian and Minear, 1958; Tizard et al, 1954). However, the nature and severity of these sensory deficits and their relationship to functional outcome have not been 
adequately described. Further investigation is required to verify the presence and extent of sensory deficits in children with static encephalopathy using a reliable assessment.

A need for the formulation of a standardized sensory assessment battery for a school-age population is clear. This battery can then be used to delineate the extent of sensory deficits in hemiplegic CP children. It would be of clinical interest to correlate the degree of sensory deficits (measured clinically and electrophysiologically) to the motor impairments noted in this population. 


\section{OBJECTIVES}

1. To formulate a sensory test battery that can be easily administered to children of school-age with neuromotor deficits and develop norms from scores of controis.

2. To evaluate the presence and extent of sensory dysfunction in hemiplegic children of school-age using clinical and electrophysiologic techniques.

3. To examine the relationship between the degree of sensory deficits and hand dysfunction in hemiplegic children. 


\section{METHODOLOGY}

\subsection{SUBJECTS}

School-aged hemiplegic children were selected. The CP children were referred by neurologists at the Montreal Children's Hospital and through the diagnostic data bank at the Shriner's Hospital. Pertinent charts were reviewed and a questionnaire was filled out by myself (hereafter referred to as Therapist $A$ ) to ensure that the inclusion criteria were met (Appendix B).

\subsubsection{Inclusion Criteria}

-the child should have a primary diagnosis of hemiplegic CP, defined as the paralysis of one side of the body (Ryerson, 1990), with no other major medical illnesses (i.e. diabetes, myopathy, neuropathy, or other peripheral/central nervous system disorders).

-the minimum requirements for language abilities should be met and are as follows:

Expressive components - at least 24-27 months *

Receptive components - at least 30-33 months *

("Bzoch-League Receptive-Expressive Emergent Language Scale, Appendix C).

-there should be at least 45 degrees of range of motion in the wrist and the fingers.

-the child's age should be between 5-18 years.

Therefore hemiplegic children with severe language deficits or significant limitations in range were not asked to participate in this study. Subjects who met all the inclusion criteria were enrolled in the study once written consent was obtained from the parent(s).(Appendix D).

\subsection{CONTROLS}

Healthy age-matched controls were recruited from schools or personal contacts. A questionnaire was filled out by the parent(s) to ensure that the child had no known neurologic impairments that may influence performance on the sensory-motor battery (Appendix E). Consent was obtained from the parents prior to testing (Appendix D). 


\subsection{EXPERIMENTAL DESIGN}

Sensory-motor investigation was carried out on a convenience sample of hemiplegic children and on 18 healthy age-matched controls, in the Neurodevelopmental Laboratory. SEP were performed in the Evoked Potential Laboratory. Both laboratories are located at the Montreal Children's Hospital. In this cross-sectional, comparative study, assessments were carried out over 2 sessions lasting approximately 2 hours each. The sensory-motor assessment was performed on each child 3 times - twice by Therapist $A$ (A1, A2) on 2 separate occasions, and once by Therapist $B$. The sensory-motor assessment lasted approximately 45 minutes. SEP were performed on both upper limbs, taking about 30 minutes. Neurological examination was carried out by a neurologist in a blind fashion in about 20 minutes. Handedness was established via a questionnaire drawn from a combination of the Crovitz-Zener and Oldfield Questionnaires (Bryden, 1977) (Appendix F).

\subsubsection{Procedures Followed}

SENSORY-MOTOR BATTERY

THERAPIST A (A1)
SENSORY-MOTOR BATTERY THERAPIST B

NEUROLOGICAL

EXAMINATION 


\subsection{SENSORY-MOTOR ASSESSMENTS}

\subsubsection{Standardization/Reliability/Validity}

A 'good' assessment is one that is easy to administer and interpret, reliable and valid (Rogers, 1987). Standardized instructions and procedures were used to perform the sensory-motor assessment. The instruments used in the battery were always constant and should not be changed, e.g. using a hairbrush instead of a toothbrusi for testing stereognosis. Cut-offs were determined based upori control values. Motor and sensory function of subjects was scored depending upon these cut-offs.

Reliability refers to the consistency or reproduceability of the results obtained by this test. Test-retest reliability, defined as stability of test scores over time, was established by having the assessor (Therapist A-A1,A2) perform the sensory-motor battery on 2 occasions on all subjects and controls. Inter-observer reliability refers to the extent to which results of a test may vary due to the differerces in scoring and testing procedures utilized by different examiners (Rogers, 1987). This was established by having 2 trained occupational therapists perform the sensory-motor battery on each child (Therapist A-A1, Therapist B).

Validity refers to the extent to which a test measures what its supposed to measure. Validity has 3 components- construct, criterion and content validity. Construct validity is defined as the appraisal of the underlying structure of the trait measured. Criterion validity can be defined as the use of test results to estimate performance on behaviour other than that assessed in the tests itself. Both construct and criterion validity could not be demonstrated because a 'gold-standard' to compare this sensory test to does not exist. Content validity refers to the extent that the test items reflect an accurate sampling of the domain being measured. This assessment is expected to have adequate content validity because items were selected from existing standardized procedures in adult sensory batteries with good validity (Jones, 1989). 


\subsubsection{Motor Assessment}

Bange of motion: Complete passive range of motion of the elbow, forearm, wrist, thumb and the index and little finger of both upper extremities was assessed (Appendix G). A goniometer was used to measure the elbow, forearm and wrist. A finger goniometer was used to measure the range of motion at the metacarpophalangeal joints of the fingers.

Muscle tone: Upper extremity tone was evaluated using the ordinal scale for tone in the Einstein Neonatal Neurobehavioural Assessment Scale (Appendix H) (Kurtzberg et al, 1979).

Grase pattern test: The Functional Evaluation of the Congenitally Anomalous Hand was used to evaluate grips and grasps (Appendix I). Basic hand function can be defined by link grip, prehensile and nonprehensile grip, and precision handling (Skerik et al, 1971). The prehensile non-manipulative grip can be divided into power and hook grips. The lateral pinch is a link grip and the non-prehensile handling grips include the palmar and the tip pinch. The hook grip was evaluated by having the subjects hold a briefcase containing the Jamar dynamometer and pinch gauge. The children were asked to hammer pegs into a pegboard to observe the power grip. Lateral pinch was observed by asking the subjects to hold a key as if they were going to open a door. Palmar pinch was observed by asking them to string a bead. Scoring follows a scale of 0 to 2 , where 2 is normal grip. 1 is object manipulation present with inappropriate grasp, 0 is no handling of object at all (Appendix G).

Grip and pinch strength: Hand grip strength can be used to test gross motor power (Jones, 1989). Grip and pınch strength were measured using a Jamar dynamometer and pinch gauge. Norms have been established for children, with an inter-rater and test :e-test reliability of 0.98 and 0.88 respectively (Ager et a',1984, Mathiowetz et al, 1986).

\subsubsection{Neurologic Examination}

A paediatric neurologist performed an examination of all subjects and controls in a blind fashion. Abnormalities in tone, deep tendon reflexes (DTRs), quality of movements and side of hemiplegia were 
noted (Appendix J). The neurologist scored the examination as normal, or abnormal (mild, moderate or severe), depending on the extent of hand disability present. The nature of lesion in subjects with CP was also noted on the basis of past medical history as obtained from charts and the careful taking of medical history when parent(s) accompanied the child. The most recent computed tomography (CT) scan and magnetic resonance imaging (MRI) of subjects were also reviewed by the neurologist to determine site and extent of injury.

\subsubsection{Somatosensory Evoked Potentials}

Becordina: The subjects and controls were placed in a comfortable supine position. The Grass Model 10 ERS system was used with G2 negativity producing an upward deflection. Both median nerves were electrically stimulated individually, via a sensory stimulator, by electrical square wave pulses delivered at $0.2 \mathrm{msec}$ duration and a rate of $4 / \mathrm{sec}$, with an intensity sufficient to obtain a thumb twitch. A stımulus isolation unit was used to minimize stimulus artifact. The electrode-patient interface was carefully cleaned with Omniprep, a mild abrasive cleaning agent. Gold cup electrodes were applied to Erb's Point (EP), over the second cervical vertebra (CII), and over the contralateral parietal scalp (Cc), using bony landmarks and the 10-20 International system of measurement. EP, CII and $\mathrm{Cc}$ were referenced to $F z$, placed in the midline of the upper forehead. The ground was attached with a strap to the proximal pc:tion of the upper limb. Arm length was measured from the point of stimulation to Erb's point. Input was amplified $100,000 \mathrm{X}$. The filters used were set at $30-3,000 \mathrm{~Hz}$. The averager was set for a sweep duration of 50 msec. Automatic sweep repetitions were set to 512. Two trials were performed and superimposed to test reproducibility of the waveform. Written records of evoked potentials were obtained on a $X-Y$ plotter.

Analysis of the SEP waveform: Analysis of the SEP waveform was done by a neurologist. The absolute latencies $(E P=N 9, C l l=N 13$, $\mathrm{C} c=$ N20, P22) were measured from the onset of stimulation to the appearance of each peak. Inter-peak latency (N13-N20) was also 
calculated. Cut-offs based on healthy control absolute and interwave latencies will be established. In this study subjects with latencies greater than the cut-offs (2.5 standard deviations greater than the mean) established, or those with flat recordings will be graded as having an abnormal SEP.

\subsubsection{Sensory Assessment}

Pressure sensitivity: This was measured using the SemmesWeinstein monofilaments (Semmes et al, 1960). The psychophysical method of limits was used to determine threshold. The filaments were applied in an ascending and a descending order. The filament first perceived in the ascending order and the filament last perceived in the descending order were noted. Three areas were tested; the volar surface of the distal phalanx of the thumb, the index and the little finger of each hand. There were 3 trials for each finger and the highest threshold obtained was used for the purpose of calculation. The hand was occluded from sight by a screen and supported in a putty (Callahan, 1984). (Appendix G).

Iwo-point discrimination (2pd). This was measured by a Diskcriminator. The distance between the 2 prongs vary from $1 \mathrm{~mm}$ to $12 \mathrm{~mm}$. Only moving $2 \mathrm{pd}$ (m2pd) was measured since it has been found to relate more closely to hand function. With vision occluded by a screen, the testing ends were moved along the fingers in a proximal-to-distal direction. One or 2 points were randomly applied to the skin surface. The subjects were asked to identify whether they felt 1 or 2 points. The smallest distance between 2 points that the subject identified without an error was taken as their m2pd threshold. Areas tested were the volar surfaces of the distal phalanx of the thumb, index and little finger. (Appendix G).

Stereognosis: It was tested via the method of visual matching Five shapes (circle, triangle, square, diamond and octagon) and 5 objects of daily use (toothbrush, tennis ball, comb 4 inches long, large cup and candy in wrapper) were presented to the subjects in random order with vision occluded. The subject was then asked to point to the object from a selection of drawings containing all 
objects. The total number of objects correctly matched out of 10 was taken as their stereognosis score. (Appendix $G$ ).

Proprioception: The child's hand was supported by the examiner's hand. With eyes occluded, the metacarpophalangeal joints of the thumb, index and little fingers were moved, holding the proximal phalanx laterally, either up or down. The subject was asked to identify the direction of movement. The number of correct responses out of 5 was scored. (Appendix G).

Directionality: A Semmes-Weinstein monofilament 4 degrees higher than the pressure threshold obtained was selected and moved along the volar aspect of the distal phalanx of the thumb, index and little finger. Order of application of the moving stimulus was random. Direction of movement of the monofilament (i.e. towards or away from the body) was required to be identified by the subject. The number of correct responses out of 5 was scored. (Appendix $G$ ).

\subsection{STATISTICS}

Independent t-tests were performed to determine if the subjects and controls were matched for age. One-way analysis of variance (ANOVA) and independent t-tests were used to examine whether age and sex had any effect on sensory function in controls, respectively. Independent t-tests were also performed on scores obtained by subjects and controls on all the sensory modalities tested by Therapist $A$ in the first testing session (A1).

Cut-offs were set at mean values $+/-2.5$ standard deviation (s.d.) for the controls. Performance (i.e. normal vs. abnormal) on the sensory-motor test in subjects was compared with data obtained in controls. Sensory function of the dominant hand in healthy controls was compared to that of the non-dominant hand by independent ttests. If dominance was found to be a factor affecting sensory performance, then cut-offs would be assigned separately for the non-dominant and dominant hand, for each sensory modality. The results were based on the results obtained by Therapist $A$ in the first testing session (A1).

Sensory function of the hemiplegic (non-dominant) hand of subjects was compared to the non-dominant hand of controls. 
Correlation of sensory function of the 'non-affected' (dominant) hand of hemiplegics and dominant hand of the healthy children was also determined. Independent t-tests were also done comparing sensory function of the 'non-affected' to that of the affected hand in hemiplegic children.

The total number of sensory modalities impaired in subjects in the affected and non-affected hand were collated. Since SEP are normally distributed cut-offs were set at mean +2.5 s.d. Therefore values greater than the cut-off values would be considered as abnormal. Subject results on the SEP recordings, neurological examination and grasp pattern scores were correlated to sensory function. Chi square analysis was performed to determine if any particular sensory modality was significantly correlated to SEP findings, neurological examination and grasp pattern scores.

Reliability was tested using the Kappa coefficient to determıne the consistency of scoring children in the study as normal or abnormal during separate testing occasions (Therapist A1/ A2 \& Therapist $A 1 / B)$. Kappa $>0.60$ was considered to be significant Intra-class correlation coefficients (ICC) were obtaıned to determine inter-rater and test-retest reliability on the raw scores obtained by the controls and subjects. ICC $>0.65$ were thought to be significant. 


\section{RESULTS}

\subsection{DESCRIPTIVE STATISTICS}

\subsubsection{Controls}

Eighteen healthy children ( 9 boys and 9 girls) were evaluated. Their ages varied from 5.25 to 16.00 years with a mean of 10.54 (s.d. 2.96) years. Fourteen children were right-handed and 4 were lefthanded (Table 1). All children were seen twice. The time difference between the two evaluations varied from 4 days to 388 days. All 18 controls were seen twice by Therapist A (A1, A2) to establish testretest reliability and 11 controls were also seen in a blind fashion by Therapist $B$ in order to establish inter-rater reliability. Somatosensory Evoked Potentials (SEP) were performed on 17/18 controls, and a neurologist blindly evaluated all 18 controls (Table 2).

\subsubsection{Subjects}

Nine children with hemiplegia ( 7 boys and 2 girls) were evaluated for the study. Subjects ranged in age from 4.33 to 18.08 years with a mean of 11.25 (s.d. 5.36) years. Subjects and controls were agematched. T-tests performed on the age of controls and subjects showed no significant difference $(p=0.66)$. Five subjects were lefthanded (right hemiplegics) and 4 were right-handed (left hemiplegics) (Table 1). The time between two sensory-motor evaluations varied from 7 to 76 days. Eight out of nine subjects were seen once by both Therapists $A$ and $B$. One subject was seen by Therapist A (A1) only once. Therapist A (A2) saw 7 subjects for a second evaluation. SEP were performed on all 9 subjects and neurological examinations were also done on all 9 subjecis (Table 2).

\subsection{NORMATIVE DATA}

Criteria for motor impairment and sensory deficits were determined based upon the results obtained from healthy controls, 
when evaluated by Therapist $A$ during the first testing session (A1). $(n=18)$.

\subsubsection{Motor Assessment Battery In The Control Group}

Range of motion: All controls had range within the normal limits as established by Scott and Trombly (1989).

Muscle tone: All of the controls obtained a score of 2 on the Einstein Neonatal Neurobehavioural Assessment Scale, i.e. normal tone.

Grase pattern test: All 18 controls scored $8 / 8$ on the grips and grasps function test (therefore a score less than 8 on this test was considered to be abnormal) in both hands (Table 3). In other words these children were able to use power grip as well as hook, lateral and palmar grasps in a correct manner.

Grip strength: Grip strength, as measured by a Jamar Dynamometer, was noted to increase significantly in the controls as a function of age, particularly so in the dominant hand. (nondominant hand: $p=0.008$, slope $=4.0, r=0.34$; domınant hand: $p=0.002$, slope $=5.28, r=0.46$ ) (Figures $1 / 2$ ).

\subsubsection{Neurological Examination In The Control Group}

Neurologic examination was performed on all 18 controls by a paediatric neurologist who was blind to the group assignment. All of the controls were found to have normal neurologic examınations All were able to use their hands in an independent manner, had no history of delayed milestones, and had normal tone, tendon jerks and reflexes.

\subsubsection{Somatosensory Evoked Potentials In The Control Group}

Seventeen out of 18 controls had SEP recordings done. N9 ranged from 7.00-10.00 msecs (mean 8.34 s.d. 0.91 msecs in the nondominant extremity and 8.40 s.d. 0.95 msecs in the domınant lımb) for both upper limbs. N13 ranged from 9.00-13.50 msecs in the nondominant upper limb (mean of 11.04 s.d. 1.23 msecs) and from 9.5013.50 msecs in the dominant upper limb (mean of 11.24 s.d. 117 msecs). N20 had latencies of $15.00-19.40$ msecs in the non-dominant 
extremity (mean of 16.85 s.d. 1.39 msecs) and of 15.00-19.00 msecs in the dominant upper limb (mean of 16.78 s.d. 1.33 msecs). Interwave latencies (N13-N20) were $5.00-7.00$ msecs on the nondominant side (mean of 5.81 s.d. 0.52 msecs) and 4.50-6.10 msecs in the dominant upper extremity (mean of 5.54 s.d. 0.35 msecs). Since SEP latency values are normally distributed, cut-offs were set at 2.5 s.d. greater than the mean for absolute and interwave latencies. Therefore values greater than the mean +2.5 s.d. would be considered as abnormal. For this study, the values of interest included N20 values (cut-off of 20.33 msecs in the non-dominant limb and 20.11 msecs in the dominant upper limb) and N13-N2O values (cut-offs of 7.11 msecs and 6.42 msecs for the non-dominant and dominant upper limbs respectively). (Table 3). Greater variability documented in $\mathrm{N} 20$ values (s.d for non-dominant=1.39, dominant=1.33) as compared to N13-N20 (s.d. for non-dominant=0.52, dominant $=0.35$ ) may be attributed to increasing arm length/body stature (thereby increasing absolute latency) as well as increasing myelination (thereby decreasing absolute latency). To assess the effect of age on SEP components, N13-N20 latencies (whose transmission site extends from brainstem to sensory cortex, and therefore would not be affected by increasing arm length) were evaluated as a function of age. No trend was seen (slope $=-0.05$, $r=0.05$ ). The slight negative slope could be due to a faster rate of conduction with increasing age due to maturation of sensory system pathways, although this was not significant. (Figure 3).

\subsubsection{Sensory Assessment Battery In The Control Group}

It was discerned that the sensory assessment battery could be easily and reliably administered in about 20 minutes. Evaluation of the effect of age and sex on all the control sensory test scores was performed by 1-way ANOVA and independent t-tests respectively. Neither of these variables were found to affect sensory performance. Independent t-tests were also done to determine if control scores on all sensory modalities, for all fingers tested, differed significantly between the dominant and non-dominant hands. Dominance was ascertained to be a significant factor in 
determining m2pd thresholds of the thumb $(T H)(p<0.05)$. Differences in m2pd thresholds and proprioception scores of the index finger (IF), directionality scores of the $\mathrm{TH}$ and stereognosis scores also approached significance (between $p=0.10$ and $p=0.15$ ). In order to make the sensory assessment battery a more sensitive tool in measuring sensory function, a more conservative approach was taken. Separate cut-offs for the dominant and non-domınant hand were used as a criteria for measuring sensory impairment in hemiplegic children.

Pressure sensitivity: Controls were assessed for pressure sensitivity using Semmes-Weinstein monofilaments, which ranged from 1.65-6.65 (logarithm of 10 times the force in milligrams required to bend the monofilament). Minimum threshold was $1.65 \mathrm{in}$ the non-dominant IF and 2.36 in all the other fingers tested Among controls, maximum threshold was 3.22 in all fingers except for the dominant $\mathrm{TH}$ (2.83) and non-dominant little finger (LF) (3.61).

The distribution of scores for all sensory modalities tested were skewed, therefore cut-offs could not be simply set by usıng mean s.d. 2.5 s.d. Given the small sample size $(N=18)$, the BienaymeChebyshev (B-C) Inequality was used to establish cut-offs. The B-C Inequality states,

"in any population or sample, no more than $(1 / z)$ squared of the observations differ from the mean by $z$ or more times the s.d. (for $z>1$ ); that is, the maximum proportion of observations in the tails of the distribution is (1/z) squared "(Olson, 1987)

Using this statistical approach we gain a greater understandıng of the relationship of s.d. to the spread of a set of observations in a small data set. The size of the s.d. will imply certain limits on how many data points can be at specific distances from the mean. The B$C$ Inequality enables us to conservatively use s.d as a measure of variability. (Olson, 1987).

Using the B-C Inequality, cut-offs were set at 2.83 in the $T H, I F$ and LF of dominant and non-dominant hand and TH of non-dominant hand. IF and LF of non-dominant hand had cut-offs of 3.22. [e.g. $1 /(2.5 \times 2.5)=0.16>1 / 18=0.06$, where $2.5=2.5$ s.d. and $18=$ number of controls]. (Table 4). Therefore scores of and above 3.22 were 
considered abnormal in the three digits tested of the dominant hand and the TH of the non-dominant hand. Scores of and over 3.61 were considered to be abnormal in the IF and LF of the non-dominant hand.

Moving two-point discrimination (m2pd): M2pd thresholds were assessed using the Disk-criminator. Minimum threshold was found to be $2 \mathrm{~mm}$ in all fingers tested and maximum threshold ranged from $3 \mathrm{~mm}$ (in both TH and dominant IF) to $4 \mathrm{~mm}$ (in non-dominant IF and LF and dominant LF). Cut-offs using the B-C Inequality were set at $3 \mathrm{~mm}$ for $T H, I F$ and LF of the dominant hand and TH of the non-dominant hand. IF and LF of the non-dominant hand had m2pd thresholds of $4 \mathrm{~mm}$. (Table 5). Thus a score $\geq 4 \mathrm{~mm}$ for both TH, and the IF and LF of the dominant hand was considered abnormal. For the IF and LF of the non-dominant hand a score of $5 \mathrm{~mm}$ and above was abnormal.

Stereognosis: Stereognosis was graded depending on the number of shapes and objects $(N=10)$ correctly identified by visual matching. Scores ranged from $8-10 / 10$ in the noll-dominant hand and 9-10/10 in the dominant hand. Cut-offs were once again established using the B-C Inequality at 9. (Table 6). Therefore a child with a score of 8 or less out of 10 was considered to have poor stereognosis.

Proprioception: Proprioception was scored depending on the number of correct responses out of five that the child gave upon movement of the child's metacarpophalangeal joint up or down. Except for the dominant IF where proprioception scores varied from 4-5/5, all children scored $5 / 5$ in proprioception on all the other fingers tested. Using the B-C inequality proprioception cut-offs were established at 5 for TH, IF and LF of both hands. (Table 7). Thus a proprioception score of 4 or less out of 5 was taken to be abnormal.

Directionality: Directionality thresholds were measured by moving a monofilament randomly up or down along the palmar aspect of the distal phalanx, and asking the child to identify the direction of movement. The monofilament was four degrees higher than pressure threshold established for that finger. Five trials were performed for each finger. Scores ranged from 3-5/5 for the dominant hand and from 4-5/5 for the non-dominant hand. Thus cutoffs were established at 4 for the TH,IF and LF of the non-dominant 
hand and at 3 for the TH, IF, and LF of the dominant hand using the BC Inequality. (Table 8). Therefore a score $\leq 3$ would be considered abnormal in fingers tested in the non-dominant hand and a score $\leq 2$ for fingers tested in the dominant hand.

\subsection{SUBJECT DATA}

Subject performance on the sensory-motor tests was rated as either normal or abnormal by comparing their scores with data obtained in controls. The results that follow were derived from the findings obtained by Therapist $A$ in the first testing session (A1). $(n=9)$.

\subsubsection{Motor Assessment Battery In The Experimental Group}

Range of motion: Three of the 9 subjects had elbow flexion contractures of $10-15^{\circ}$, on the affected side.

Muscle tone: All 9 hemiplegic children had increased tone of the affected upper extremity (i.e. a score of 3-moderately hypertonic, on the Einstein Neonatal Neurobehavioural Assessment Scale).

Grasp pattern test: The grips and grasps hand function evaluation showed that 3 hemiplegic children (3/9) had normal grasp patterns All 3 were right hemiplegics $(\mathrm{RH})$. Of the 6 hemiplegic children with abnormal grasp patterns, 1 child showed mild-moderate impairment (i.e. a score of $5-7 / 8$ ) and 5 subjects had moderate-severe involvement (score of $0-4 / 8$ ). In summary, 6/9 hemiplegic children had abnormal scores on the test of hand function of which 4 were $\mathrm{LH}$ and 2 had RH (Table 9).

Grip strength: All subjects had decreased grip strength on the affected hand when compared to the non-affected hand. In $3 / 8$ subjects grip strength was decreased by $80 \%-100 \%$ on the affected side. In 3 other children the affected limb had a decreased strength of $50 \%-80 \%$ when compared to the non-affected hand. The remaining two children had minimal differences (approximately 20\%) in the grip strength of the affected vs. the non-affected hand. In one child, this sub-test could not be performed due to lack of cooperation. 


\subsubsection{Neurological Examination In The Experimental Group}

Neurological evaluation was performed by a neurologist who was blind to the neurological status of the 9 subjects The extent of independent hand and finger function was rated as normal, mild, moderate or severe. Mild involvement was present if some independent finger movement was possible, moderate impairment was present if voluntary grasp was present without individual finger movements, and severe deficits were present if no voluntary grasp was possible. The neurologist rated $3 / 9$ subjects as having mild hemiplegia, 1 had a rating of moderate hand involvement, and 4 with severe hemiplegia. All $4 \mathrm{LH}$ were found to have severe involvement of the affected side (hand) whereas the $5 \mathrm{RH}$ had varying degrees of hemiplegia (Table 10).

The nature of the brain lesion of all subjects was explored by the neurologist by carefully examining the medical charts and taking a history when parent(s) accompanied the child. The most recent CT scans of $8 / 9$ hemiplegic children and the MRI in 3 subjects were also evaluated by the neurologist to determine the extent and location of the lesion. The neurologist concluded that 3 hemiplegics suffered anoxic-ischemic insults, 2 of which occurred in the prenatal period, to deep structures as well as the frontal and parietal lobes of the affected hemisphere. Another 3 subjects had porencephaly, 1 of prenatal orıgin (fronto-central area involved), while in the other 2 , it appeared to occur in the neonatal period (only deep structures involved in 1 and diffuse frontal, parietal and occipital involvement in the other child). In the remaining 3 subjects, 1 had cerebral atrophy (suggesting a watershed infarct), another pachygyria (sylvain and calcarine fissures involved), and the last subject showed evidence of cerebral atrophy (deep structures as well as frontal and parietal lobe lesions) (Table 11).

\subsubsection{Somatosensory Evoked Potentials In The Experimental Group}

Three out of the 9 hemiplegic children assessed had flat N2O waveforms on the affected side. Two had increased N2O latencies with delayed conduction times (N13-N20) and diminished amplitudes 
on the affected side (Figure 4). Four subjects had normal SEP bilaterally. All $4 \mathrm{LH}$ had abnormal SEP recordings. Three had flat N2O waveforms and 1 had an increased N20 latency along with an increased N13-N2O interwave latency. Four of the $5 \mathrm{RH}$ had normal SEP and 1 had an abnormal SEP, with an increased latency and a delay in conduction time (Table 12).

\subsubsection{Sensory Assessment Battery In The Experimental Group}

Sensory function on the affected side: Independent $t$-tests demonstrated that significant sensory deficits are present on the affected side of hemiplegic children across all modalities, as compared to the non-dominant hand of controls. Pressure sensitivity of the $T H$ and IF, directionality of LF, m2pd of IF and LF, proprioception of all fingers (all significant at $p<0.05$ ) and stereognosis $(p<0.001)$ were significantly impaired (Table 13), (Figures 6-10).

In the affected hand, 1 hemiplegic child had no sensory deficit on modalities tested, whereas all the other children demonstrated deficits of 1 or more sensory modalities. There were 3 individuals who had 1, 2, and 3 modalities affected respectively. Three subjects had impairment of all 5 sensory modalities of the affected hand. Due to short attention span and lack of vooperation, 2 hemiplegic subjects were unable to complete the 5 sub-tests but had $2 / 3$ and 2/2 sensory modalities impaired. (Table 16).

Pressure Sensitivity was measured by the Semmes-Weinste monofilaments. Using the cut-offs as established on control data, it was found that 3 of the 7 hemiplegic children assessed had increased pressure sensitivity thresholds on the affected side (Table 18). Dellon's Disk-criminator was used to assess m2pd. Four out of 7 subjects (2/9 were un-cooperative) had increased m2pd thresholds on the affected side (Table 18). Accurate identification of shapes and objects was used to test stereognosis. This modality showed maximal impairment in these children. Seven of the 9 hemiplegic children had astereognosis of the affected hand (Table 18). Proprioception was tested by evaluating the subject's 
awareness of joint position in space. This modality was frequently impaired as well. Six of 9 hemiplegics had impaired proprioception on the affected side (Table 18). The subject's ability to correctly distinguish the direction in which a monofilament was moved was used to test directionality. Five of 8 hemiplegic children had impaired directionality (Table 18).

Sensory function on the 'nen-affected' side: Significant sensory deficits were also present on the so called "non-affected' side of hemiplegic children, as illustrated by independent t-test results, when compared to sensory test scores obtained in the dominant hand of the controls. Pressure sensitivity $(p<0.05)$ (Figure 5$), \quad m 2 p d$ threshold $(p<0.01)$, proprioception $(p<0.05)$ and stereognosis $(p<0.01)$ on the 'non-affected' hand showed significant sensory deficits in the hemiplegic children (Table 14).

In the so called 'non-affected' hand, $1 \mathrm{LH}$ had 4 sensory modalities impaired, and 3 children had involvement of 1 sensory modality. Due to short attention span, 2 hemiplegic children were unable to complete their sessions, but had $1 / 2$ sensory modalities tested impaired. The remaining 3 had no sensory involvement present. (Table 17). Thus $6 / 9$ (66\%) hemiplegic children had 1 or more sensory modalities impaired on the 'non-affected' side.

Two children were not evaluated completely due to poor cooperation. . On the 'non-affected' side, 2 children (both LH) had increased pressure sensitivity, therefore demonstrating bilateral impairment in pressure sensitivity. None of the hemiplegic children showed impaired mapd threshold on the 'non-affected' side. Three out of 9 subjects had impaired stereognosis, as well as a deficit in proprioception. Only 1 child (LH) had a deficit in directionality on the 'non-affected' side. (Table 19).

Comparison of sensory function on the affected and 'non-affected' hands in the experımental group by t-tests, displayed no significant differences between the 2 sides (Table 15).

Sensery vs. SEP findings: Scores obtained on all sensory modalities were correlated to SEP findings using chi-square analysis. SEP recordings were graded as 0 for a flat N20 wave, 1 if the N2O waveform was of increased latency and 2 if the SEP 
waveform latency was within normal limits. Children in the study were scored on all sensory modalities tested. Using cut-off values as determined by control data, children were scored as 1 if their scores were within normal limits for that sensory modality, and 2 for an abnormal score in 1 or more of the fingers tested, for that sensory modality. Chi-square analysis showed that SEP were significantly correlated with pressure sensitivity of the IF in both dominant and non-dominant hands, as well as the LF of the nondominant hand $(p<0.05)$. M2pd of the $T H, I F$ and $L F$ of the nondominant (affected) hand were found to significantly correlate to SEP on the same side $(p<0.05)$. Finally, directionality of the nondominant LF was also determined to have a significant correlation to SEP recordings.

Sensory findings across the 3 fingers tested were collated for the dominant and non-dominant hand, for all 5 sensory modalities. Thus, sensory impairment on even 1 site would correspond to a score of 2 (abnormal sensory function) for that particular sensory modality in the same hand, whereas a score of 1 implied normal sensory function in all 3 fingers tested. This sensory modality score was correlated with SEP findings. Once again SEP (on the affected side) were found to be significantly correlated to pressure sensitivity and $\mathrm{m} 2 \mathrm{pd}$ thresholds of the non-dominant hand $(p<0.05)$

Sensory function was assessed clinically by the Sensory Assessment Battery as well as using an electrophysiologic technique (SEP). Interestingly, this study shows that SEP do not always correlate positively with clinical sensory findings Four hemiplegic children with SEP abnormalities (flat N2O or delayed conduction time) had severe sensory deficits. However one child with an increased N2O latency had no sensory deficits Conversely, the 4 children with normal SEP had mild (1/4) or moderate (3/4) sensory impairment (Table 20).

Sensory vs. motor assessment results: Motor function was assessed by neurological examination and grasp patterns. Neurological examination was scored as 0 for a severe hemiplegia (no voluntary grasp), 1 for moderate impairment (voluntary grasp present, but no individual finger movements), 2 for mild hemiplegia 
(some individual finger movements), and 3 for a normal neurologic evaluation. Grasp patterns (hand function sub-test) were graded as 0 for a moderate-severe impairment (score of $0-4 / 8$ ), 1 for a mildmoderate impairment $(5-7 / 8)$, and 2 for normal grasp patterns (8/8).

Both methods of assessing motor function (neurological examination and grasp patterns) corresponded $100 \%$ using 2 independent examiners. Therefore all 3 of the children classified as mild hemiplegia by the neurologist had normal grasp patterns (as determined by Therapist $A 1)$. The child with moderate hemiplegia demonstrated mild-moderate impairment of grasp patterns, and the remaining 5 subjects with severe hemiplegia displayed moderatesevere grasp pattern involvement (Table 20).

Chi-square analysis was performed to determine if scores on all sensory modalities were significantly correlated to motor function, as assessed by neurological examination and grasp pattern scores. Neurological examination and grasp patterns were not ascertained to be significantly correlated to scores on any sensory modality tested. Collated sensory modality scores for the dominant and non-dominant hand was also correlated to neurological examination and grasp pattern scores, using chi-square analysis. Once again, no significant relationship was determined between any sensory modality and motor function.

When sensory ability of the hand was correlated to its motor function, 2 interesting findings were observed Firstly, sensory function as determined by performance on the Sensory Assessment Battery did not correlate with motor function. For example, in Table 20 , child \#4 had severe hemiplegia with normal sensory function. Conversely, child \#9 had mild hemiplegia with moderate sensory impairment. Thus sensory function scores did not correlate to motor function in $3 / 9$ hemiplegic children. In contrast, SEP correlated well with findings on the motor ability of the hand. All 5 hemiplegic children with SEP abnormalities (flat or increased conduction time) were graded by the neurologist as having severe impairment of their affected upper extremity. Furthermore they all had a rating of moderate to severe impairment in grasp patterns, as determined by Therapist A (A1). The other 4 subjects had normal SEP. One of these 
4 children was graded as moderately impaired and had a mild to moderate deficit in grasp patten. The other 3 with normal SEP had mild hemiplegia and normal grasp patterns (Table 20).

\subsection{RELIABILITY}

Both test-retest and inter-rater reliability of subjects and controls were assessed. Therapist $A$ tested the controls and subjects with the sensory-motor assessment battery on two separate occasions (A1, A2). Time between $A 1$ and $A 2$ varied between 4-388 days (mean of 202.72 s.d. 160.72 days) for controls and between 7-76 days (mean of 26.43 s.d. 25.61 days) for subjects Test results in this battery, as obtained by $A 1$ were also compared to results obtained by Therapist $B$, thereby establishing interobserver reliability.

The kappa coefficient was performed on scores obtained by controls and subjects in the non-dominant and dominant hand $A$ Kappa Coefficient $>0.60$ was considered significant. Pressure sensitivity scores in the IF showed $K=1.00$ for Therapist $A$ and $B$; and $K=0.78$ for Therapist $A(A 1, A 2)$. M2pd scores in the TH and IF of the non-dominant hand reported a $\mathrm{K}=1.00$ for hoth test-retest and interrater reliability. Test-retest relıability as determıned for stereognosis was $K=0.88$ for the non-dominant hand and $K=064$ for the dominant hand. Proprioception scores showed a test-retest reliability of $\mathrm{K}=0.78$ and $\mathrm{K}=0.64$ for the non-dominant and dominant TH respectively. Inter-rater reliability measured for proprioception showed $K=1.00$ and $K=0.63$ for the $T H$ in the non-dominant and dominant hands respectively. Directionality scores when measured for test-retest reliability showed $K=1.00$ for the $T H$ and LF of the dominant hand, and $K=0.67$ for the $\mathrm{TH}$ and $\mathrm{LF}$ of the non-dominant hand (Tables $21 \& 22$ ) Percentage agreement on sensory battery scores between raters and $A 1$ and $A 2$ assessments varied from $81 \%$ $100 \%$ for the non-dominant/affected hand and $75-100 \%$ for the dominant/non-affected' hand (Tables 21 \&22).

Intraclass Correlation Coefficients (ICC) were performed on the raw scores obtained by the children in the study on tests of sensory function on their dominant and non-dominant hands $A n$ ICC $>0.65$ 
was regarded as significant. Pressure sensitivity scores showed significant inter-rater reliability for all fingers tested in both the dominant and non-dominant hands. Significant test-retest reliability of pressure sensitivity of the non-dominant hand and the TH of the dominant hand was also determined. M2pd thresholds of nondominant showed significant reliability between Therapist $A$ and $B$, and $I F$ and LF also showed significant test-retest reliability. M2pd of the LF of the dominant hand was ascertained to have an ICC $=0.66$ for Therapist $A(A 1, A 2)$. Both test-retest and inter-rater reliability as determined for stereognosis in the non-dominant hand had $I C C=0.87$ and 0.74 respectively. $I C C$ for proprioception scores of the $\mathrm{TH}$ of the non-dominant hand was 0.89 . ICC for proprioception values on all the other fingers tested could not be computed due to no variation in proprioception scores obtained, however the scores indicate excellent reliability. In the non-dominant hand, directionality scores showed significant test-retest reliability for the TH and good inter-rater reliability for the IF (Tables 23 \&24).

Therefore overall, test-retest and inter-rater reliability was determined to be excellent across all modalities. 


\section{DISCUSSION}

\subsection{Formulation of A Reliable Paediatric Test Battery For Children With Neuromotor Deficits}

The standardized sensory battery formulated in this study assesses 5 sensory modalities in school-age children. Only children with a certain level of language function (expressive component of 24-27 months and receptive component of 30-33 months) can be reliably assessed using this test. This ensures that instructions are understood. Only non-verbal cues or simple responses are required (e.g. stereognosis was executed by visual matching), in order to minimize the expressive language requirement for test administration. All subjects were expected to answer either yes or no (pressure sensitivity testıng), 1 or 2 (m2pd evaluation) and up or down (proprioception and directionality assessments)

No provisions were made for behavioural factors which could influence the reliability of the test scores. Two hemıplegıc children in the study exhibited short attention spans and a high level of distractibility. These 2 children had difficulty completıng the test battery. Both of them were able to perform the sub-tests stereognosis and proprioception, which are of short duration and therefore require a shorter attention span They had great difficulty concentrating on and completing the other sensory tests; in particular, pressure sensitivity with the Semmes-Weinstein monofilaments. Thus behavioural difficulties (poor attention span, distractibility) in children to be assessed is an important factor to be considered in the clinical application of this assessment. The time required for sensory evaluation was approximately 20 minutes in the controls. The hemiplegic children required about $35-40$ minutes to complete the same test. About an hour was needed to assess each of the 2 children with attention deficits.

When comparing the sensory thresholds of the dominant and nondominant hand in controls, important (significant or approaching significance) differences were found for some modalities. Thus a more conservative approach was adopted; cut-offs for each sensory modality were derived separately for the dominant and non-dominant 
hand. Interestingly, cut-offs in the dominant hand were lower (higher sensitivity) for pressure sensitivity and m2pd. This could possibly be explained by both the nature or nurture theories. Propounders of the 'nature' theory postulate that an individual's genetic make-up is chiefly responsible for their traits and abilities. Based on this, theorists presume that the dominant hemisphere would mature earlier (i.e. may be more myelinated), thus sensory input would be more rapidly projected rostrally on the dominant side. Conversely, developmentalists supporting the 'nurture' theory propose that the environment and an individual's experience are the chief trait building factors. These nurture theorists would advocate that the greater sensitivity of the dominant hand may be due to its greater degree of sensory experience. Stereognosis and proprioception had the same cut-offs for both hands. However for stereognosis, the mean was higher (greater sensitivity) with less variability (s.d. $=0.38$ ) in the domınant hand as compared to the nondominant hand $(s . d .=0.62)$. Thus even though there was no difference between stereognosis values obtained for both hands overall, dominance did show some effect in individual cases. On the other hand directionality scores of the dominant hand were lower (decreased sensitivity) as compared to the non-domınant hand. One may speculate that this could be due to habituation, since the dominant hand is used more often. In summary, this study shows that there are important differences between the sensitivity of the dominant and non-dominant hand. Therefore, when performing this sensory assessment, hand dominance should be taken into consideration, as cut-off values could vary.

Age and sex were found to have no effect on the control values obtaıned for all sensory modalities. Thus it was not necessary to compute cut-off values for different age groups (within 5-16 years) or based on the sex of the child.

All sensory modalities were found to be reliable in identifying a child with sensory abnormality when assessed by chance-corrected Kappa (0.00-1.00). M2pd had the highest test-retest $(K=1.00$ for all fingers), and inter-rater reliability $(K=1.00$ for the $T H$ and $I F$, and $K=0.65$ for the LF) for the dominantaffected hand. Directionality had 
the lowest test-retest reliability $(K=0.00$ for the $I F$ and $K=0.67$ for the $\mathrm{TH}$ and $(F)$, as well as the lowest Kappa scores for inter-rater reliability ( $K=0.00$ for all fingers), for the dominant/affected hand. A reason for low directionality scores could be the slippage of the filament, while applying it on the digit. Another factor to be considered could be the mathematical layout of the Kappa test. This can be seen on considering the degree of percentage agreements between assessors (inter-rater reliability), and the 2 assessments performed by Therapist $A(A 1, A 2)$ (test-retest reliability). It was observed to be $\geq 75 \%$. All sensory modalities were found to have a good overall reliability between the actual scores obtained (ICC > 0.65 ) in both the non-dominant vs. the affected and dominant vs. 'non-affected' hands. Several of the proprioception ICC scores were 0.00 . This can be accounted for by the absence of any variability in the raw scores Thus this sensory battery was found to be a reliable tool for assessing sensory function.

The battery formulated for this study includes 5 sensory modalities which convey varying types of somesthetic information regarding the shape, size and texture of the surface that is being explored. These sensory cues are crucial for optimal hand performance. We selected m2pd because it has been found to be a good predictor of hand function (Dellon, 1984). Movement of our hands over an object is the primary means of obtaining somesthetic information about the object (Essick and Whitsel, 1985) Thus, directionality, pressure sensitivity, stereognosis and proprioception are important components of hand function. Hence these sensory modalities have important diagnostic utility, particularly in children with motor impairment and therefore should be included in a sensory assessment battery.

Though paediatric test batteries which evaluate components of sensory function exist, several problems with their use are evident. These assessments are not applicable to children with neuromotor deficits, due to the high degree of language ability and motor function required to complete the assessment. Furthermore, some of these assessments do not have norms for a school-age population (e.g. MAP, SCSIT) (DeGangi, 1987; Stowers and Huber, 1987). Scoring 
the QNST is subjective due to the qualitative nature of the observations that have to be made. The SCSIT requires that the examiners be specially trained in administering and scoring the assessment, and therefore is not widely available for clinical use. This test has also been found to have poor reliability. Modalities which are not objective and precise measures of sensation were assessed in these assessments (i.e. graphesthesia and kinesthesia). None of these test batteries assess m2pd, pressure sensitivity or directionality of touch, which are important components of hand function. All of these problems have been addressed in my study.

In a paediatric population the sensory system may still be immature, therefore norms may differ as compared to adult norms. We chose to develop specific norms, which would be applicable to a school-aged population. In this study it was determined that grip strength increases as a function of age and sex. This observation has been previously documented in several studies which have assessed and provided norms for grip strength for adults as well as children (Mathiowetz et al, 1984; Mathiowetz et al, 1985; Mathiowetz et al, 1986).

This study is unique in providing cut-off paediatric values for pressure sensitivity, m2pd and stereognosis. However a larger sample size would be necessary to generalize the results.

Cut-offs for pressure thresholds of most fingers were determined as 2.83 in this study. However, results obtained established nondominant IF and LF cut-off pressure thresholds at 3.22. In the adult literature, pressure thresholds above 2.83 are considered to be abnormal (Bell, 1984). Thus some adult pressure threshold values were found to differ slightly, when compared to my study's paediatric values. This may be due to the physical immaturity of the developing sensory system in children. Therefore it is very important to have a separate set of criteria with which to gauge sensory function in children.

In this study, control m2pd values are presented, as well as criteria or cut-offs to determine abnormal m2pd in the 5-16 year age group. Any $2 \mathrm{pd}$ values in the literature pertain to static 2pd, with a general agreement that $\mathrm{m} 2 \mathrm{pd}$ thresholds are lower and more 
closely related to hand function, than static $2 \mathrm{pd}$ thresholds (Dellon and Kallman, 1983; Dellon, 1984; Louis et al, 1984). Thus m2pd cutoffs obtained in miy study may be a good predictor of hand function. Though many researchers have specified a normal range of $2 \mathrm{pd}$ values, no specific cut-offs have been described which would help delineate a normal from an abnormal 2pd threshold (Jones, 1989). Norms have been provided for $2 p d$ for different regions of the upper and lower limbs (Gellis and Pool, 1977; Louis et al, 1984; Nolan, 1983). Studies by both Gellis and Pool (1977), and Louis et al (1984) evaluated $2 \mathrm{pd}$ in a wide age range of healthy controls (7-86 years; 4-92 years respectively). It was discovered that the lowest thresholds are obtained in the third decade. However no actual $2 \mathrm{pd}$ values were provided.

The objects chosen in our battery to assess stereognosis were all familiar to children, therefore differing from objects used in evaluations previously. Objects that may give other sensory cues to the children, enhancing their object identification (e.g. temperature cues - metal spoon and key), were not selected. Norms for stereognosis for a set of objects have been reported in the literature. However, the set of objects used in different studies have varied. thus norms available for a given set of objects are based on small samples (Jones, 1989)

No norms/control values have been established in the literature for proprioception and directionality. Therefore this study has provided a set of cut-off values for 5 sensory modalities, that can be used in a paediatric assessment of sensory function

The sensory assessment battery reliably assessed sensory performance in hemiplegic children, in spite of their motor involvement. This assessment battery could be applied to other children with central nervous system dysfunction, provided that they meet the criteria for administration (1.e language and behavioural limits).

All the children that participated in this study enjoyed the sensory-motor assessment, especially the evaluation of stereognosis, proprioception and grasp patterns However assessment of pressure sensitivity and directionality using the 
Semmes-Weinstein monofilaments was found to be tedious and uninteresting by most of the children. Therefore to maximize the cooperation of the child, it is recommended that those sub-tests which are fun and interesting to children should be performed first, with the more tedious sub-tests being left to the end. A larger number of controls are required to establish norms. Therefore, results from this study should be generalized with caution because of the small sample size.

In summary, a reliable sensory-motor battery was established for use with a paediatric population of school-age. Furthermore, this battery requires minimal language and motor skills, and therefore can be easily administered to children with neuromotor deficits. For example, it would be of interest to study the type and extent of sensory deficits that exist in other forms of static encephalopathy At present, it is being used to evaluate the effect of selective dorsal rhizotomy coupled with intensive post-operative therapeutic intervention, on sensory, as well as, locomotor function in CP children with spastic diplegia.

\subsection{Evaluation of Sensory Function In Hemiplegic Children Using Clinical And Electrophysiological Techniques}

In my study, sensory function of the affected hand of hemiplegic subjects was found to be significantly altered across all modalities $(P<0.05)$. A significant impairment of sensory function of the so called 'non-affected' hand was also determined $(p<0.05)$. Interestingly, no significant differences between a hemiplegic child's 2 hands were found. Based on these findings one can conclude that significant sensory deficits are present on both the affected and 'non-affected' hand in hemiplegic children

Descriptive statıstics were used to establish cut-off values for detecting normal/abnormal sensory function in our study sample. Results obtained suggest that $88 \%$ of hemiplegic children tested had involvement of one or more sensory modalities on their affected side. (Table 16) It was observed that $77.8 \%$ of children had impairment in stereognosis, $66.7 \%$ in proprioception, and $62.5 \%$ in directionality (Table 18). M2pd and pressure sensitivity were also 
impaired to a lesser extent $(57.1 \%$ and $42.9 \%$ respectively). These results are similar to the findings of studies by Hohman et al (1958), Monfraix et al (1961) and Tachdjian and Minear (1958), in which stereognosis and proprioception were 2 of the modalities found to be most often impaired in hemiplegics. Many o: the hemiplegic children assessed in my study also had varying degrees of sensory involvement on the 'non-affected' side (6/9). The 'nonaffected' side exhibited deficits of stereognosis $(3.3 .3 \%)$, proprioception (66.7\%), pressure sensitivity $(286 \%)$ and directionality $(14.3 \%)$. Interestingly no deficits in $\mathrm{m} 2 \mathrm{pd}$ were detected on the non-affected side (Table 19). Few studies have reported similar findings. Brown et al (1989) found that hemiplegic children, aged $3-13$ years may exhibit bilateral visuo-motor impairment, as tested by the accuracy, and reaction tıme taken to point to targets. Monfraix et al, (1961) reported the presence of sensory agnosia on the non-affected side. Brown et al (1987) concluded that their study results were based on the presumption that the 'non-affected' hand in hemiplegics has normal function, even though they pointed out that this might not be so. The findings of my study provide evidence that sensory dysfunction is present in both hands in hemiplegic children Therefore, studies whose results were based upon the use of the 'non-affected' side as a control are misleading (Tachdjian and Minear, 1958, Tizard et al, 1954).

Hence, clinicians dealing with children with hemiplegia should always be aware of the possible presence of bilatc,al sensory impairment. Diagnostic procedures in this population should include assessment of bilateral sensory function The hand that has so far been considered as 'non-affected' is actually a less affected hand (1 shall from now on refer to it as such).

The CT scan and MRI have been found to be of great value in defining the site and extent of focal and multifocal lesions of the cerebrum, intracranial hemorrhage and lesions of periventricular white matter (Volpe, 1987). It was therefore of interest to correlate clinical sensory findings on the sensory-motor assessment battery, to the location and extent of the injury as revealed by the CT scans and MRI impressions. 
CT scan and MRI findings correlated well to sensory function. Those children with severe sensory deficits were determined to have diffuse and multifocal involvement of the frontal and parietal areas of the cortex, and of the deep underlying structures (Table 11subjects \#1,2,3,5). The diffuse bilateral nature of the lesions of these 4 children was also reflected in the varying degrees of sensory deficits (1/4-mild, 2/4 moderate and 1/4 had severe sensory deficits) that they manifest on the contralateral hand.

The 3 children with moderate sensory impairment, demonstrated a lesser degree of cortical involvement on CT scan and MRI findings (Table 11-subjects $\# 6,7,9$ ). Interestingly the MRI findings of 1 of them showed a remote watershed infarct (i.e. a parasagittal injury in the posterior parietal-temporal region). This probably encompasses the posterior parietal lobule. Studies have shown that excision of the posterior parietal lobule leads to astereognosis, spatial disorientation of the contralateral side of the body (Roland, 1987) and an impairment in object manipulation (Pause and Freund, 1989). This was reflected by subject test scores on the clinical sensory battery, in which the subject showed a bilateral deficit of stereognosis with difficulty in object manipulation. Thus, this study reveals that clinical sensory findings reflected the site and extent of cortical injury, as evaluated by the CT scan and MRI.

One child had a unilateral mild impairment of sensation on the affected side. These findings correlated to $C T$ scan and MRI readings, which ascertanned a mild diffuse lesion involving the Sylvain fissure and extending posteriorly into the Calcarine fissure (Table 11subject \#8). Thus, SII which is located in the upper bank of the lateral sulcus may have been involved. SIl has been implicated in sensory-motor integration of the ipsilateral and contralateral sides of the body (Roland, 1987). This finding is demonstrated in this child as a deficit of stereognosis, which is a sensory modality requiring exploratory movements of the fingers to help identify the object.

Lastly, 1 child was determined to have normal bilateral sensory function. This child's CT scan revealed a porencephaly of only the deep central area with no cortical involvement (Table 11-subject \#4). Therefore, radiological findings on the CT scan and MRI 
correlated with the degree of sensory involvement as determined by the sensory assessment battery. However, information regarding the type of modalities affected obtained by the sensory-motor battery, is not provided by neuromaging techniques.

In my study, the sensations maximally affected were stereognosis and proprioception. Stereognosis has been determined to be a higher function, which is a composite of several integrated primary sensory inputs (Maugiere et al, 1983). Both proprioception and tactile discrimination of shape and texture of an object play an important role in object identification (stereognosis) Studies have determined that $S$ I modulates both proprioception and stereognosis on the contralateral side of the body (Carlson, 1981; Corkın et al, 1970; Darian-Smith et al, 1982; Roland, 1987; Semmes and Turner, 1977). The posterior parietal lobule is also involved in the modulation of stereognosis (Roland, 1987). Both stereognosis and proprioception involve a motor component as part of their testing procedure. (i.e. manipulation of objects for stereognosis, joint movement for proprioception) Lesions of $S 11$, which is responsible for the sensory-motor integration of the ipsilateral and contralateral sides of the body (Roland, 1987), could also result in impaired proprioception and stereognosis. Injury to any part of the somesthetic cortex would have a greater probability of leading to a deficit of stereognosis and proprioception, than the other more passive sensory inputs which are mediated only by SI (Pause and Freund, 1989) Thus, these 2 sensations are more prone to be affected and this is clearly illustrated in this study

An interesting observation of this study was that left hemiplegics $(\mathrm{LH})$ demonstrated an overall greater degree of bilateral sensory impairment, as compared to right hemiplegics $(\mathrm{RH})$. The children with right hemiplegia had primarily bilateral imparrment of stereognosis, with other modalities being impaired to a lesser degree on the affected side. This could also be linked to the site and extent of injury. The $\mathrm{RH}$ tended to have a mild diffuse involvement and focal lesions as compared to the left hemiplegics who had more diffuse lesions involving deep structures and large areas of the cortex (particularly the parietal and frontal lobes). Nass and Koch, 
(1991) postulated that intrahemispheric reorganization could also be the reason for most of the $\mathrm{RH}$ being reported as having a lesser degree of sensory deficits. In the case of congenital injury, the right hemisphere is more capable of taking over many of the functions of the left hemisphere. Therefore $\mathrm{RH}$ (children with lesions of the left hemisphere) would have a better chance of recovery. Another factor to be considered is that the uncrossed pyramidal tract is better developed on the right side. Therefore in the case of left cortical injury, it would probably be able to exert greater control on the right hand, as compared to its control on the left hand after right hemisphere injury (Brown et al, 1987). Thus left brain damaged children $(\mathrm{RH})$ may fare better functionally than children with right brain damage (LH) (Brown et al, 1987; Nass and Koch, 1991).

In my study sensory function was assessed by 2 methods-a clinical evaluation of sensory function using the sensory assessment battery, and through SEP findings. This is the first study that has examined the relationship between clinical and electrophysıological sensory evaluations, whereby the method of assessment is well documented and the correlation between assessments is statistically analyzed.

The dorsal column-medial lemniscal pathway mediates discrete touch, proprioception, pressure sensitivity, vibration, and regulates skillful activity (Martin and Jessel, 1991). SEP are a non-Invasive method of examining the integrity of the dorsal column-medial lemniscal system. SEP waveforms are an especially valuable tool in a paediatrıc population due to their high reliability (Cracco, 1989).

Studies reviewed in the literature have evaluated the relationship between SEP and clinical sensory function (Karnaze et al, 1987; Laget et al, 1976; Larson et al, 1966; Liberson, 1966; Maugiere et al, 1983; Williamson et al, 1970; Wong et al, 1982; Zeman and Yiannikas, 1989) and concluded that SEP have a good relationship to neurological impairment (muscle weakness and sensory loss), as well as, functional outcome. These studies have not described the specific methods by which the clinical sensory loss was measured and the degree of sensory deficits detected. Statistical computations of the relationship between SEP findings and sensory 
function were also not clearly stated. Some of these studies have not specified which sensory modalities were assessed (Williamson et al, 1970; Wong et al, 1982). Studies by Laget et al (1976). Liberson (1966), Maugiere et al (1983) and Williamson et al (1970), gauged sensory loss on the integrity of only 1 sensory modality. Even though one sensory modality may correlate to SEP findings other sensory modalities may or may not be affected.

In contrast to findings in the literature, this study demonstrated that SEP findings did not correlate to results obtained on the sensory battery for $5 / 9$ subjects. An abnormal SEP (flat N20 wave or increased N13-N20 interwave latency) was found to be a better predictor of severe sensory deficits ( $4 / 5$ children), than a normal SEP in predicting normal sensory function ( $0 / 4$ children) (Table 20), there were many false negatives. Children with a lesser degree of sensory impairment would be missed if SEP were the only tool being used for the evaluation of sensory function. This finding is supported by Giblin's (1964) investigations on 42 patients, wherein he noted that in spite of sensory deficits being correlated to SEP alterations in most of his subjects, 7 patients with moderate to severe sensory deficits had normal SEP recordings and 1 subject with normal sensory function had an abnormal SEP. Therefore SEP by themselves should not be used as a diagnostic tool for predicting sensory loss The standardized sensory assessment formulated in this study on the other hand has been shown to be a sensitive assessment that can detect even mild sensory loss. Thus the sensory battery would be invaluable in the diagnostic work-up of the child with hemiplegia The SEP could be used as an adjunct to the sensory assessment battery since it provides us with additional information on the integrity of the dorsal column-medial lemniscal system

The results described above may be explained by the generators of the SEP waveform. Though precise delineation of SEP generator sites is controversial, the N20 wave is thought to be generated, by most, from the posterior bank of the central sulcus (Fagan et al, 1987) Thus, the SEP cortical generators are located anterior to the transmission sites (S I, S II and posterior parietal lobule) of the sensory modalities tested. Therefore, one may speculate that the 
SEP waveform would not necessarily determine sensory function. If a lesion is focal and situated posterior to the central sulcus the SEP findings would not reveal this cerebral injury. This assumption is reinforced by the findings of this study. The 4 hemiplegic children with mild-moderate sensory involvement on the clinical sensory assessment and normal SEP recordings had lesions of the more posterior parieto-occipital regions (Tables 11 \& 20-subjects \#6-9).

A statistically significant correlation between the SEP recordings and the modalities of pressure sensitivity and $m 2 p d$ was found on the affected hand of subjects $(p<0.05)$. This could reflect the proximity of the SEP N20 generator site to the area of transmission of the sensations of pressure and $\mathrm{m} 2 \mathrm{pd}$, the $\mathrm{S}$ 1-postcentral gyrus and depths of the central sulcus (Dykes, 1978).

Hence, many interestıng and clinically relevant findings came to light in this study. The need to assess sensory function as a part of a comprehensive evaluation of a $C P$ child should once again be emphasized. Not only were sensory deficits present on the affected side, the so-called 'non-affected' hand was also determined to have sensory deficits. Thus no evaluation of a child with central nervous system disorder is complete without the assessment of sensory function bilaterally. Our findings suggest that the extent of sensory loss correlates with the site and extent of cortical injury in the subjects, as demonstrated by CT/MRI findings. In contrast SEP findings did not clearly relate to the extent of sensory loss in the subjects, especially in those with mild-moderate sensory deficits. The clinical sensory battery was shown to be a more accurate measure of the extent and nature of sensory function.

\subsection{Relationship between Motor and Sensory Function in} Hemiplegic Children

Sensory function was assessed in this study using the clinical sensory battery and SEP. Clinical sensory function was graded as normal, mild, moderate or severe depending on the number of sensory modalities involved. SEP recordings were either within the normal range, or the N13-N20 interwave latency was delayed, or the N20 wave was flat. 
Motor function was assessed by a neurologist and by the performance of subjects on a grasp pattern test. The neurologist graded the subjects on their ability to voluntarily grasp and release. The children were either rated as normal, or as having mild, moderate or severe hemiplegia. Four grasp patterns were evaluated Depending on their scores, subjects were graded as having normal grasp patterns, or as demonstrating a mild-moderate or moderatesevere grasp pattern impairment.

Both tests of motor function correlate perfectly ( $\mathrm{e}$ children with severe hemiplegia had moderate-severe impairment of grasp patterns, moderate hemiplegia corresponded to mild-moderate grasp pattern dysfunction, children with mild hemiplegia had normal grasp patterns). This suggests that both the neurological examination and test of grasp pattern were valid tools for evaluatıng motor function

When clinical sensory function was correlated to each individual's motor ability in this study some interesting findings were documented. Firstly, there was no significant correlation between the clinical sensory status and elther the neurological examination or the grasp pattern scores (Table 20). For example, subject \#4 had a severe degree of hemiplegia and moderate-severe impairment of grasp pattern but displayed no sensory loss Conversely subject \#9 had mild hemiplegia and normal grasp patterns, and demonstrated moderate sensory impairment on the sensory assessment battery Thus, the motor capability of a child does not appear to mirror his/her sensory function Therefore, clınicians while evaluatıng children with hemiplegia should bear in mind that a child demonstratıng a mild degree of hemiplegia might have significant sensory loss. Conversely, a child with severe motor involvement may not have significant sensory deficits.

Few studies performed in the past have studied the relationship between sensory deficits and motor function in $\mathrm{CP}$ children (Kenney,1966; Twitchell,1966). Methods of administering and scoring the sensory assessments in these studies were unreliable and often not clearly specified. Thus this is the first study of its kind to analyze the relationship between clinical sensory and motor function. 
SEP have been reported to be a sensitive electrophysiological tool for predicting functional outcome in $\mathrm{CP}$ children and high-risk newborns (Logan et al, 1991; Majnemer et al, 1987). Laget et al (1976) reported that SEP were a better predictor of motor impairment than EEGs, in their study of 43 hemiplegic children. In the current study it was found that SEP findings correlated well with motor impairment as determined by neurological examination and test of grasp patterns. Thus those children with abnormal SEP (flat or increased latency of the $\mathrm{N} 20$ waveform) were rated as severely impaired on neurological examination, and obtained a rating of moderate-severe impairment of grasp pattern. Normal SEP waveforms also correlated to mild impairment or normal hand function on the neurological evaluation and test of grasp patterns (Table 20). This supports the findings by Majnemer et al (1987) that SEP can be used as a predictor of motor outcome in newborns with static encephalopathy. The results of this study showed SEP to be a better predictor of motor function than of sensory ability. This could be related again to the generator site of the cortical component of the SEP waveform. Therefore the SEP findings would correspond with motor function due to the close proximity of its generator site to the motor strip. Those hemiplegic children with more diffuse, multifocal and frontal involvement as displayed by the CT/MRI fındings (Table 11-subjects \#1-5) had both SEP abnormalities and severe motor function impairment. The 4 children with normal SEP and mild-moderate involvement of motor ability had brain insults to the more posterior parieto-occipital region (Table 11-subjects \#69). This study supports the view that SEP whose cortical components are generated close to the precentral gyrus reflect the degree of motor dysfunction and severity of $\mathrm{CP}$

In conclusion, study results demonstrated that both methods of motor function assessment (neurological examınation and grasp pattern evaluation) correlated perfectly. Sensory evaluation using the standardized clinical sensory assessment battery displayed that sensory deficits did not correlate to motor function. However, SEP findings were positively related to motor ability, which is likely to 
be related to the $\mathrm{N} 20$ generator site and its close proximity to the motor strip.

\subsection{Conclusions}

Cerebral Palsy (CP) is one of the foremost childhood handicapping conditions. Though aberrant motor control and posture are its primary characteristics, a review of the literature suggests that sensory deficits in CP children are present as well. Though many valid and reliable motor assessments have been developed, the state of the art is quite lacking for sensory assessments for school-aged children. Thus, the need for a study to assess sensory function in CP children using rigorous methodological and statistical procedures was evident.

In view of the findings in previously reported studies, this study brings a new assessment to light A standardized sensory assessment battery for school-aged children was formulated This assessment evaluates 5 sensory modalities (pressure sensitivity, m2pd, stereognosis, proprioception and directionality) in a reliable fashion. Though some of the sensory sub-tests used in this battery exist as separate entities, it is for the first tıme that they have been combined as components of a sensory battery A standardized set of simple instructions and method of administration were used during test performance. The assessment was short and interesting for the children, thus being ideal for a paediatric population During performance of this assessment the therapist used objective, quantitative measures e.g. Semmes-Weinstein monofilaments and Disk-criminator, which have been established to have a high reliability Those modalities which were closely linked to effective hand manipulation were carefully selected. A mınımum level of language ability was required. Provisıons for measuring behaviour were not made, however this would be advisable in the future

This study determined paediatric cut-off values for the modalities of proprioception and directionality for the first time Though other studies have described norms for pressure sensitivity, m2pd and stereognosis, either the norms were for an adult population or the tests were not administered in a standardized 
fashion. Thus this study provides paediatric cut-off values for pressure sensitivity, $\mathrm{m} 2 \mathrm{pd}$ and stereognosis, measured in a standardized manner. While administering the sensory assessment, hand dominance was found to affect performance. Control data obtained demonstrated that cut-off values used to detect abnormal sensory function differed for the non-dominant and dominant hands. Analysis of tost-retest and inter-rater reliability revealed that all the sensory modalities tested had strong reliability, both in controls and hemiplegic children. Both age and sex had no effect on control values for any sensory modality. This is the first assessment of its kind that will enable the clinician to objectively assess sensory function in school-age children with a variety of disabling conditions, such as static encephalopathy.

Assessment of sensory function in the hemiplegic child revealed several clinically relevant features. The affected hand of $8 / 9$ hemiplegic children had sensory deficits Stereognosis (7/9), proprioception (6/9) and directionality (5/8) were the primary modalities impaired. Sensory deficits were not only observed on the affected side but the so-called 'non-affected' side also showed significant sensory impairment (6/9). Proprioception and stereognosis were the modalities maximally affecter in the 'lessaffected' hand. Thus clınıcians should bear in mind that when evaluating the functional integrity of a child with hemiplegia, a bilateral sensory assessment should be performed.

This study is unique in that it is the first study to ascertain sensory function in hemiplegic children using both a clinical sensory battery and SEP. It was observed that SEP did not correlate to sensory ability as determined clinically. It was hypothesized that this could be due to the relationship between the cortical site of SEP generation and the region of sensory transmission and modulation in the cortex. Findings on the CT scan correlated well with clinical sensory findings. Hence, the clinical sensory test provides more extensive and meanıngful information to clinicians regarding the extent (number of modalities imparred) and nature (the modalities impaired) of sensory involvement.as compared to SEP. This study reinforces the fact that SEP should not be used as a 
indicator of sensory loss, and furthermore does not reflect the integrity of any 1 sensory modality.

Finally, this was the first study that examıned the relationship between sensory and motor function in hemiplegic children using standardized methods of assessment. Sensory function was assessed clinically and by an electrophysiologic technique (SEP). Motor function was graded depending upon the child's performance on a standardized grasp pattern test and on neurological examination of motor ability. It was observed that both tests of motor function agree perfectly. Sensory function as assessed by the sensory assessment battery, did not correlate with motor function. However SEP were found to be a good predictor of motor function. This could be due to the location of the N20 generator site of the SEP potential, which lies in close proximity to the motor strip, but is somewhat more distant from the sensory transmission sites

A standardized sensory battery which can be utilized to assess school-age children was formulated. This assessment can be applied to children enveloping a wide range of diagnostic categories (e g orthopaedic, neurologic, and arthritic/rheumatic) including significant neuromotor impairment, and therefore has important clinical utility. Normative data for the sensory assessment battery needs to be improved by increasing the number of children evaluated, so that it can be applied with confidence in the clinical setting Norms on the lower extremity (i.e. big toe) would have clinıcal value as well. This study has reinforced the importance of carefully evaluating sensory function in hemiplegic childrer, and therefore a sensory assessment should be an integral part of the comprehensive evaluation of a hemiplegic child. The sensory assessment battery could be applied to children with other patterns of brain injury causing statıc encephalopathy i.e. periventricular leukomalacia with clinical evidence of spastic diplegia, children with inultifocal, diffuse (selective neuronal necrosis) or parasagittal cerebral injury manifested clinically as spastic quadriplegia

Not only does CP have an astounding effect on the functional ability of the individual, it also has a profound effect on the life of the immediate family. Therapeutic intervention and prognosis deperid 
upon accurate appraisal of abilities and disabilities (Banus, 1979). $C P$ is known to be a severely handicapping disorder. Currently, treatment is focused on the identified motor deficits, with the underlying sensory deficits often being overlooked. Sensory impairments, if present, could have a debilitating effect on the overall functional capabilities of the CP child. The importance of the intact sensory system on hand function has been repeatedly demonstrated by both experimental and clinical studies (Bolanos et al, 1989; Curry and Exner, 1962; Van Buskirk and Webster, 1955; Westling and Johansson, 1984). Thus, an objective and reliable sensory assessment battery may prove to be invaluable in the formulation of therapeutic goals, as well as gauging the efficacy of ongoing treatment, ultimately maximizing the handicapped child's functional potential. 


\begin{tabular}{|l|l|l|l|l|l|l|l|l|}
\hline & \multicolumn{4}{|c|}{ AGE (years) } & \multicolumn{3}{c|}{ SEX } & \multicolumn{3}{c|}{ HANDEDNESS } \\
\hline & $5-8$ & $8-12$ & $12-16$ & $16-20$ & FEMALE & MALE & LEFT & RIGHT \\
\hline $\begin{array}{l}\text { CONTROLS } \\
N=18\end{array}$ & 4 & 10 & 4 & 0 & 9 & 9 & 4 & 14 \\
\hline $\begin{array}{l}\text { SUBJECTS } \\
N=9\end{array}$ & 3 & 2 & 2 & 2 & 2 & 7 & 5 & 4 \\
\hline
\end{tabular}

Table 1: Descriptive statistıcs

\begin{tabular}{|c|c|c|c|c|c|c|c|c|c|}
\hline & \multicolumn{4}{|c|}{$\begin{array}{l}\text { Time in days between Assess. } \\
\qquad 1 \& 2\end{array}$} & \multirow[t]{2}{*}{ A 1} & \multirow[t]{2}{*}{ A 2} & \multirow[t]{2}{*}{ B } & \multirow[t]{2}{*}{ SEP } & \multirow[t]{2}{*}{$\begin{array}{l}\text { Neuro. } \\
\text { Exam. }\end{array}$} \\
\hline & 0.100 & $\begin{array}{lll}1 & 0 & 1- \\
2 & 0 & 0\end{array}$ & $\begin{array}{l}201- \\
300\end{array}$ & 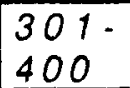 & & & & & \\
\hline Controls & 8 & 0 & 1 & 9 & 18 & 18 & 11 & 17 & 18 \\
\hline Subjects & 7 & 0 & 0 & 0 & 9 & 7 & 8 & 9 & 9 \\
\hline
\end{tabular}

Table 2: Descriptive statistıcs

KEY:

Assess. $\quad$ :Assessment

A 1 :Therapist A- Assessment 1

A 2 :Therapist A- Assessment 2

B :Therapist B

SEP :Somatosensory Evoked Potentials

Neuro. Exam. :Neurological Examination 


\begin{tabular}{|l|c|c|c|c|c|c|c|c|}
\hline & \multicolumn{6}{|c|}{ NON-DOMINANT UPPER LIMB } & \multicolumn{4}{c|}{ DOMINANT UPPER LIMB } \\
\cline { 2 - 9 } & N9 & $\mathbf{N 1 3}$ & N20 & $\begin{array}{c}\text { N13- } \\
\text { N20 }\end{array}$ & N9 & N13 & N20 & $\begin{array}{c}\text { N13- } \\
\text { N20 }\end{array}$ \\
\hline $\begin{array}{l}\text { RANGE: } \\
\text { m in- } \\
\text { max }\end{array}$ & $\begin{array}{c}7.00 \\
\text { to } \\
10.00\end{array}$ & $\begin{array}{c}9.00 \\
\text { to } \\
13.50\end{array}$ & $\begin{array}{c}15.00 \\
\text { to } \\
19.40\end{array}$ & $\begin{array}{c}5.00 \\
\text { to } \\
7.00\end{array}$ & $\begin{array}{c}7.00 \\
\text { to } \\
10.00\end{array}$ & $\begin{array}{c}9.50 \\
\text { to } \\
13.50\end{array}$ & $\begin{array}{c}15.00 \\
\text { to } \\
19.00\end{array}$ & $\begin{array}{c}4.50 \\
\text { to } \\
6.10\end{array}$ \\
\hline Mean & 8.34 & 11.04 & 16.85 & 5.81 & 8.40 & 11.24 & 16.78 & 5.54 \\
\hline s.d. & 091 & 1.23 & 1.39 & 0.52 & 0.95 & 117 & 1.33 & 035 \\
\hline $\begin{array}{l}\text { Mean + } \\
\text { 2.5 s.d. }\end{array}$ & 10.62 & 14.12 & 20.33 & 7.11 & 10.78 & 14.17 & 20.11 & 642 \\
\hline
\end{tabular}

$\mathrm{n}=17$.

Table 3: Somatosensory evoked potential control values

KEY:

min-max :Mınimum and maximum values obtained

s.d. :Standard deviation 


\begin{tabular}{|l|c|c|c|c|c|c|}
\hline & \multicolumn{3}{|c|}{ NON-DOMINANT HAND } & \multicolumn{3}{c|}{ DOMINANT HAND } \\
\hline & THUMB & INDEX F. & LITTLE F. & THUMB & INDEX F. LITTLE F. \\
\hline $\begin{array}{l}\text { RANGE: } \\
\text { m in- } \\
\text { max }\end{array}$ & $\begin{array}{c}2.36 \\
\text { to } \\
3.22\end{array}$ & $\begin{array}{c}1.65 \\
\text { to } \\
3.22\end{array}$ & $\begin{array}{c}2.36 \\
\text { to } \\
3.61\end{array}$ & $\begin{array}{c}2.36 \\
\text { to } \\
2.83\end{array}$ & $\begin{array}{c}236 \\
\text { to } \\
322\end{array}$ & $\begin{array}{c}236 \\
\text { to } \\
322\end{array}$ \\
\hline $\begin{array}{l}\text { Mean } \\
\text { s.d. }\end{array}$ & 2.51 & 2.53 & 2.57 & 2.51 & 2.58 & 261 \\
\hline $\begin{array}{l}\text { Mean + } \\
\text { 2.5 s.d. }\end{array}$ & 3.06 & 3.36 & 3.37 & 2.96 & 3.18 & 321 \\
\hline Cut-off & 2.83 & 3.22 & 3.22 & 283 & 283 & 283 \\
\hline
\end{tabular}

$n=18$

Table 4: Pressure sensitivity control values

KEY:

INDEXF. :Index finger

LITTLEF. :Little finger

min-max Minımum and maximum values obtained

s.d. :Standard deviation

The Semmes-Weinstein monofilaments are graded instruments for detecting pressure sensitivity thresholds They range from 165 to 6.65. The monofilament whose value was closest to the mean +25 s.d. numerical value without going over was chosen as the cut-off Therefore for the four fingers for whom the mean $+25 \mathrm{sd}$ value was between 2.83 and 322 monofilaments the cut-off was set at 2.83. Thus for those fingers a pressure threshold of 283 and below can be considered normal. For the two fingers whose mean $+25 \mathrm{~s} \mathrm{~d}$ value lay between 3.22 and 361 cut-off was set at 3.22 . 


\begin{tabular}{|c|c|c|c|c|c|c|}
\hline & \multicolumn{3}{|c|}{ NON-DOMINANT HAND } & \multicolumn{3}{|c|}{ DOMINANT HAND } \\
\hline & THUMB & INDEX F. & LITTLE $F$. & THUMB & INDEX F. & LITTLE F. \\
\hline $\begin{array}{l}\text { RANGE: } \\
\text { mir - } \\
\text { max }\end{array}$ & $\begin{array}{l}2.00 \\
t c \\
3.00 \\
\end{array}$ & $\begin{array}{c}2.00 \\
\text { to } \\
4.00 \\
\end{array}$ & $\begin{array}{c}2.00 \\
10 \\
4.00\end{array}$ & $\begin{array}{l}3.00 \\
t 0 \\
3.00 \\
\end{array}$ & $\begin{array}{c}2.00 \\
t 0 \\
3.00 \\
\end{array}$ & $\begin{array}{l}2.00 \\
\text { to } \\
4.00 \\
\end{array}$ \\
\hline Mean & 2.72 & 2.50 & 2.83 & 3.00 & 2.28 & 2,83 \\
\hline s.d. & 0.46 & 0.62 & 0.51 & 0.00 & 0.46 & 0.51 \\
\hline $\begin{array}{l}\text { Mean }+ \\
2.5 \text { s.d. }\end{array}$ & 3.87 & 4.05 & 4.11 & 3.00 & 3.43 & 411 \\
\hline Cut-off & $3 \mathrm{~mm}$ & $4 \mathrm{~mm}$ & $4 \mathrm{~mm}$ & $3 \mathrm{~mm}$ & $3 \mathrm{~mm}$ & $3 \mathrm{~mm}$ \\
\hline
\end{tabular}

$n=18$

Table 5: Moving two-point discrimination control values

\begin{tabular}{|c|c|c|}
\hline & NON-DOMINANT HAND & DOMINANT HAND \\
\hline $\begin{array}{l}\text { RANGE: } \\
\text { min-max }\end{array}$ & $\begin{array}{c}800 \\
\text { to } \\
10.00\end{array}$ & $\begin{array}{c}9.00 \\
\text { to } \\
10.00\end{array}$ \\
\hline Mean & 9.56 & 9.83 \\
\hline s.d. & 0.62 & 0.38 \\
\hline Mean - 2.5 s.d. & 8.01 & 8.88 \\
\hline Cut-off & 9 & 9 \\
\hline
\end{tabular}

$n=18$

Table 6: Stereognosis control values

KEY:

INDEX F. : Index finger

LITTLE F. : Little finger

min-max :Minimum and maximum values obtained

s.d. $\quad$ :Standard deviation 


\begin{tabular}{|l|c|c|c|c|c|c|}
\hline & \multicolumn{3}{|c|}{ NON-DOMINANT HAND } & \multicolumn{3}{c|}{ DOMINANT HAND } \\
\hline & THUMB & INDEX F. & LITTLE F. & THUMB & INDEX F. & LITTLE F. \\
\hline $\begin{array}{l}\text { RANGE: } \\
\text { min- } \\
\text { max }\end{array}$ & $\begin{array}{c}5.00 \\
\text { to } \\
5.00\end{array}$ & $\begin{array}{c}5.00 \\
\text { to } \\
5.00\end{array}$ & $\begin{array}{c}500 \\
\text { to } \\
5.00\end{array}$ & $\begin{array}{c}5.00 \\
\text { to } \\
5.00\end{array}$ & $\begin{array}{c}400 \\
\text { to } \\
5.00\end{array}$ & $\begin{array}{c}5.00 \\
\text { to } \\
5.00\end{array}$ \\
\hline s.d. & 5.00 & 5.00 & 5.00 & 5.00 & 489 & 5.00 \\
\hline $\begin{array}{l}\text { Mean - } \\
\text { 2.5 s.d. }\end{array}$ & 0.00 & 0.00 & 0.00 & 000 & 032 & 000 \\
\hline Cut-off & 5 & 5 & 5 & 5 & 5 & 5 \\
\hline
\end{tabular}

$n=18$

Table 7: Proprioception control values

\begin{tabular}{|c|c|c|c|c|c|c|}
\hline & \multicolumn{3}{|c|}{ NON-DOMINANT HAND } & \multicolumn{3}{|c|}{ DOMINANT HAND } \\
\hline & THUMB & INDEX $F$. & LITTLE F. & THUMB & INDEX F. & LITTLE F \\
\hline $\begin{array}{l}\text { RANGE: } \\
\text { m in- } \\
\text { max }\end{array}$ & $\begin{array}{l}4.00 \\
10 \\
5.00\end{array}$ & $\begin{array}{c}4.00 \\
10 \\
5.00\end{array}$ & $\begin{array}{c}4.00 \\
10 \\
500\end{array}$ & $\begin{array}{c}300 \\
10 \\
500\end{array}$ & $\begin{array}{c}300 \\
10 \\
500\end{array}$ & $\begin{array}{c}300 \\
10 \\
500\end{array}$ \\
\hline Mean & 4.67 & 4.56 & 4.67 & 4.39 & 444 & 450 \\
\hline s.d. & 0.49 & 0.51 & 0.49 & 070 & 062 & 062 \\
\hline $\begin{array}{l}\text { Mean - } \\
2.5 \text { s.d. }\end{array}$ & 3.45 & 4.05 & 3.45 & 2.64 & 289 & 295 \\
\hline Cut-off & 4 & 4 & 4 & 3 & 3 & 3 \\
\hline
\end{tabular}

$n=18$

Table 8: Directionality control values

KEY:

INDEX F. :Index finger

LITTLE F. : Little finger

min-max :Minimum and maximum values obtained

s.d.

:Standard deviation 


\begin{tabular}{|c|c|c|c|c|c|}
\hline \multicolumn{2}{|c|}{$\begin{array}{c}\text { NORMAL } \\
(8 / 8)\end{array}$} & $\begin{array}{c}\text { MILD-MOD. IMPAIR. } \\
(5-7 / 8)\end{array}$ & \multicolumn{2}{c|}{$\begin{array}{c}\text { MOD.-SEV. IMPAIR. } \\
(0-4 / 8)\end{array}$} \\
\hline \multicolumn{2}{|c|}{$3 / 9$} & \multicolumn{2}{|c|}{$1 / 9$} & \multicolumn{2}{c|}{$5 / 9$} \\
\hline LH & RH & LH & RH & LH & $R H$ \\
\hline $0 / 4$ & $3 / 5$ & $0 / 9$ & $1 / 5$ & $4 / 4$ & $1 / 5$ \\
\hline
\end{tabular}

Table 9: Grasp pattern scores of hemiplegic children

KEY:

MILD-MOD. IMPAIR. : Mild to moderate impairment of hand function

MOD.-SEV IMPAIR. : Moderate to severe impairment of hand function
UH
: Left Hemıplegic
PH
: Right Hemiplegic

\begin{tabular}{|c|c|c|c|c|c|c|c|}
\hline \multicolumn{2}{|c|}{ NORMAL } & \multicolumn{2}{c|}{$\begin{array}{c}\text { MILD } \\
\text { INVOLVEMENT }\end{array}$} & \multicolumn{2}{c|}{$\begin{array}{c}\text { MODERATE } \\
\text { INVOLVEMENT }\end{array}$} & \multicolumn{2}{c|}{$\begin{array}{c}\text { SEVERE } \\
\text { INVOLVEMENT }\end{array}$} \\
\hline \multicolumn{2}{|c|}{$0 / 9$} & \multicolumn{2}{|c|}{$3 / 9$} & \multicolumn{2}{|c|}{$1 / 9$} & \multicolumn{2}{c|}{$5 / 9$} \\
\hline LH & $\boldsymbol{R H}$ & $\mathbf{L H}$ & $\boldsymbol{R H}$ & $\mathbf{L H}$ & $\boldsymbol{R H}$ & $\mathbf{L H}$ & $\boldsymbol{R H}$ \\
\hline $0 / 4$ & $0 / 5$ & $0 / 4$ & $3 / 5$ & $0 / 4$ & $1 / 5$ & $4 / 4$ & $1 / 5$ \\
\hline
\end{tabular}

Table 10 Neurological examination results of hemiplegic children

KEY:

LH: Left Hemiplegic

$\mathrm{RH}$ : Rıght Hemıplegic 


\begin{tabular}{|c|c|c|}
\hline SUBJECTS & $\begin{array}{l}\text { TYPE OF } \\
\text { LESION }\end{array}$ & SITE AND EXTENT OF LESION \\
\hline$\# 1$ (LH) & anoxic-ischemic & $\begin{array}{l}\text { In right MCA territory Involv. of deep struct } \\
+ \text { temporal, parietal \& Irontal lobes }\end{array}$ \\
\hline$\# 2(\mathrm{LH})$ & cerebral atrophy & $\begin{array}{l}\text { Right hemisphere atrophy involving parietal } \\
\& \text { frontal lobes }+ \text { deep struct involv }\end{array}$ \\
\hline$\# 3(\mathrm{LH})$ & porencephaly & $\begin{array}{l}\text { Microcranıa + diffuse lesion involv fronto } \\
\text { central region }\end{array}$ \\
\hline$\# 4$ (LH) & porencephaly & $\begin{array}{l}\text { Substance loss in the deep central area + } \\
\text { enlargement of right ventricle }\end{array}$ \\
\hline$\# 5(\mathrm{RH})$ & anoxic-Ischemic & $\begin{array}{l}\text { Diffuse lesion involv frontal/central } \\
\text { processing dysfunction }\end{array}$ \\
\hline \#6 (RH) & anoxic-ischemic & $\begin{array}{l}\text { Left parietal/occipital thinning of brain } \\
\text { mantle + enlargement of lateral ventricle }\end{array}$ \\
\hline$\# 7$ (RH) & porencephaly/abscess & $\begin{array}{l}\text { Involv of right frontal lobe + left panieto } \\
\text { occipital region }\end{array}$ \\
\hline$\# 8(\mathrm{RH})$ & pachygyria & $\begin{array}{l}\text { Involv of the sylvain fissure up to the } \\
\text { calcarine fissure }+ \text { mild diffuse hemiatrophiy }\end{array}$ \\
\hline$\# 9(\mathrm{RH})$ & cerebral atrophy & $\begin{array}{l}\text { Developmental smallness of left hemisphere } \\
\text { remote left watershed infarct }\end{array}$ \\
\hline
\end{tabular}

Table 11: CT scan and MRI findings in hemiplegıc subjects

KEY:

UH .Left hemiplegia

PH :Right hemiplegia

MCA :Middle cerebral artery

Involv :involvement

Struct structures 


\begin{tabular}{|c|c|c|c|c|c|}
\hline \multicolumn{2}{|c|}{ NORMAL SEP } & \multicolumn{2}{c|}{$\begin{array}{c}\text { DELAYED } \\
\text { CONDUCTION TIME }\end{array}$} & \multicolumn{2}{c|}{ FLAT N20 WAVE } \\
\hline \multicolumn{2}{|c|}{$4 / 9$} & \multicolumn{2}{|c|}{$2 / 9$} & \multicolumn{2}{c|}{$3 / 9$} \\
\hline LH & RH & LH & RH & LH & $R H$ \\
\hline $0 / 4$ & $4 / 5$ & $1 / 4$ & $1 / 5$ & $3 / 4$ & $0 / 5$ \\
\hline
\end{tabular}

Table 12: Somatosensory evoked poteritial recordings on the affected side in hemiplegic children

KEY:

SEP : Somatosensory Evoked Potentials

LH · Left hemiplegic Children

A1 : Right Hemiplegic Children 


\begin{tabular}{|c|c|c|c|c|c|}
\hline & $\begin{array}{c}\text { PRESS. } \\
\text { SENS. }\end{array}$ & M2PD. & STEREO. & PROP. & DIR. \\
\hline TH & 0.024 & 0.095 & \multirow{2}{*}{0.001} & 0.003 & 0.069 \\
\hline IF & 0.036 & 0.015 & & 0.020 & 0.362 \\
\hline LF & 0.139 & 0.028 & & 0.020 & 0.031 \\
\hline
\end{tabular}

Table 13:P values based on sensory function of the affected side of subjects as compared to the non-dominant side of controls.

\begin{tabular}{|c|c|c|c|c|c|}
\hline & $\begin{array}{c}\text { PRESS. } \\
\text { SENS. }\end{array}$ & M2PD. & STEREO. & PROP. & DIR. \\
\hline TH & 0.050 & 0.009 & \multirow{3}{*}{0.008} & 0.017 & 0.598 \\
\hline IF & 0.082 & 0.605 & 0380 & 0487 \\
\hline LF & 0.371 & 0500 & & 0110 & 0500 \\
\hline
\end{tabular}

Table 14:P values based on sensory function of the 'non-affected' side of subjects as compared to the dominant side of controls

\begin{tabular}{|c|c|c|c|c|c|}
\hline & $\begin{array}{c}\text { PRESS. } \\
\text { SENS. }\end{array}$ & M2PD. & STEREO. & PROP. & DIR. \\
\hline TH & 0.227 & 0296 & \multirow{3}{*}{0.122} & 0200 & 0809 \\
\hline IF & 0.212 & 0.080 & 0182 & 1000 \\
\hline LF & 0.521 & 0.121 & & 0321 & 0490 \\
\hline
\end{tabular}

Table 15.P values based on sensory function of the affected side of subjects as compared to their 'non-affected' side

\section{KEY:}

PRESS. SENS. :pressure sensitivity

M2PD.

STEREO

:moving two-point discrimination

PROP.

DIR.

TH.

IF

LF ;stereognosis

:proprioception

:directionality

:thumb

:index finger

little finger

Significant $p$ values $(p<0.05)$ are represented in italics 


\begin{tabular}{|c|c|}
\hline $\begin{array}{c}\text { \# SENSORY MODALITIES } \\
\text { INVOLVED }\end{array}$ & $\begin{array}{c}\text { \# HEMIPLEGIC } \\
\text { CHILDREN }\end{array}$ \\
\hline $0 / 5$ & $1 / 7$ \\
\hline $1 / 5$ & $1 / 7$ \\
\hline $2 / 5$ & $1 / 7$ \\
\hline $3 / 5$ & $1 / 7$ \\
\hline $4 / 5$ & $0 / 7$ \\
\hline $5 / 5$ & $3 / 7$ \\
\hline
\end{tabular}

Table 16: Number of sensory modalities impaired on the affected hand of hemiplegıc children

\begin{tabular}{|c|c|}
\hline $\begin{array}{c}\text { \# SENSORY MODALITIES } \\
\text { INVOLVED }\end{array}$ & $\begin{array}{c}\text { \# HEMIPLEGIC } \\
\text { CHILDREN }\end{array}$ \\
\hline $0 / 5$ & $3 / 7$ \\
\hline $1 / 5$ & $3 / 7$ \\
\hline $2 / 5$ & $0 / 7$ \\
\hline $3 / 5$ & $0 / 7$ \\
\hline $4 / 5$ & $1 / 7$ \\
\hline $5 / 5$ & $0 / 7$ \\
\hline
\end{tabular}

Table 17: Number of sensory modalities impaired on the 'nonaffected' hand of hemiplegic children

\section{FOOTNOTE:}

Two subjects had short attention span and were uncooperative and therefore could not be tested on the complete test battery. On the affected side 1 child had impairment in 2 modalities of the 3 tested (2/3) and the other child had deficits in both the sensory modalities tested (2/2). On the 'non-affected' side both of these 2 hemiplegic children had 1 modality impaired of the 2 sensory modalities tested (1/2). 


\begin{tabular}{|c|c|c|c|c|c|c|c|c|c|}
\hline \multicolumn{2}{|c|}{ PRESS. } & \multicolumn{2}{c|}{ M2PD. } & \multicolumn{2}{c|}{ STEREO. } & \multicolumn{2}{c|}{ PROP. } & \multicolumn{2}{c|}{ DIR. } \\
\hline \multicolumn{2}{|c|}{$3 / 7$} & \multicolumn{2}{|c|}{$4 / 7$} & \multicolumn{2}{|c|}{$7 / 9$} & \multicolumn{2}{c|}{$6 / 9$} & \multicolumn{2}{c|}{$5 / 8$} \\
\hline$L H$ & $R H$ & $L H$ & $R H$ & $L H$ & $R H$ & $L H$ & $R H$ & $L H$ & $R H$ \\
\hline $2 / 3$ & $1 / 4$ & $2 / 3$ & $2 / 4$ & $3 / 4$ & $4 / 5$ & $3 / 4$ & $3 / 5$ & $2 / 3$ & $3 / 5$ \\
\hline
\end{tabular}

Table 18: Sensory involvement on the affected hand of hemiplegic children

\begin{tabular}{|c|c|c|c|c|c|c|c|c|c|}
\hline \multicolumn{2}{|c|}{ PRESS. } & \multicolumn{2}{c|}{ M2PD. } & \multicolumn{2}{c|}{ STEREO. } & \multicolumn{2}{c|}{ PROP. } & \multicolumn{2}{c|}{ DIR. } \\
\hline \multicolumn{2}{|c|}{$2 / 7$} & \multicolumn{2}{|c|}{$0 / 7$} & \multicolumn{2}{|c|}{$3 / 9$} & \multicolumn{2}{c|}{$3 / 9$} & \multicolumn{2}{|c|}{$1 / 7$} \\
\hline $\mathbf{L H}$ & $\boldsymbol{R H}$ & $\mathbf{L H}$ & $\boldsymbol{R H}$ & $\mathbf{L H}$ & $\boldsymbol{R H}$ & $\mathbf{L H}$ & $\boldsymbol{R H}$ & $\mathbf{L H}$ & $\boldsymbol{R H}$ \\
\hline 2.3 & 0.4 & 0.3 & 0.4 & 14 & 25 & 24 & 15 & 13 & 04 \\
\hline
\end{tabular}

Table 19: Sensory involvement on the 'non-affected' hand of hemiplegıc children

KEY:

PRESS. : Pressure Sensitivity Threshold

M2PD. : Moving Two-Point Discrimination Threshold

STEREO. : Stereognosis Threshold

PROP. : Proprioception Threshold

DIR. : Directionality Threshold

LH : Left Hemiplegic

RH : Right Hemiplegic 


\begin{tabular}{|c|c|c|c|c|}
\hline SUBJECTS & SEP & NEURO. EXAM. & MOTOR FN. & SENSORY FN. \\
\hline$\# 1$ (LH) & Flat & Severe & Mod-severe & Severe \\
\hline \#2(LH) & Flat & Severe & Mod-severe & Severe \\
\hline \#3(LH) & Flat & Severe & Mod-severe & Severe $^{*}$ \\
\hline \#4 (LH) & Inc. latency & Severe & Mod-severe & Normal \\
\hline$\# 5(R H)$ & Inc. latency & Severe & Mod-severe & Severe \\
\hline$\# 6(R H)$ & Normal & Moderate & Mild-mod & Moderate \\
\hline$\# 7(R H)$ & Normal & Mild & Normal & Moderate* \\
\hline$\# 8(R H)$ & Normal & Mild & Normal & MIId \\
\hline$\# 9(R H)$ & Normal & Mild & Normai & Moderate \\
\hline
\end{tabular}

Table 20 . Summary of results of the experimental group

\section{KEY:}

SEP

NEURO. EXAM

MOTOR FN.

SENSORY FN

$\mathrm{LH}$

PH

MOD

INC. LATENCY

MODERATE*
- Somatosensory Evoked Potentials

: Neurological Examination

- Motor Function

: Sensory Function

: Left Hemiplegic

: Right Hemiplegic

: Moderate involvement

: Increased N13-N20 interwave latency

: Moderate involvement, however not all sensory

modalities assessed due to short attention span

Motor function was graded normal, or with mild, moderate or severe motor impairment depending on grasp pattern.

Score of $8 / 8 \quad$ :Normal

$5.7 / 8$ :Mild-Moderate deficit

$0-4 / 8$ :Moderate-Severe deficit

Sensory Function was graded normal, or with mild, moderate or severe sensory impairment depending on the number of sensory modalities involved.

Modalities affected $0 / 5$ :Normal deficit

1/5 :Mild deficit

2-3/5 :Moderate deficit 


\begin{tabular}{|c|c|c|c|c|c|c|c|c|c|c|}
\hline \multirow{2}{*}{$\begin{array}{c}\text { KAPPA } \\
\& \\
\% \\
\text { AGREE. }\end{array}$} & \multicolumn{2}{|c|}{$\begin{array}{l}\text { PRESS. } \\
\text { SENS. }\end{array}$} & \multicolumn{2}{|c|}{$\begin{array}{c}\text { M2PD } \\
\text { THRESH. }\end{array}$} & \multicolumn{2}{|c|}{$\begin{array}{l}\text { STEREO. } \\
\text { SCORES }\end{array}$} & \multicolumn{2}{|c|}{$\begin{array}{l}\text { PROP. } \\
\text { SCORES }\end{array}$} & \multicolumn{2}{|c|}{$\begin{array}{l}\text { DIR. } \\
\text { SCORES }\end{array}$} \\
\hline & $A 1 / A 2$ & $A 1 / B$ & $A 1 / A 2$ & $A 1 / B$ & $\mathrm{~A} 1 / \mathrm{A} 2$ & $A 1 / B$ & $\mathrm{~A} 1 / \mathrm{A} 2$ & $A 1 / B$ & $\overline{A 1 / A 2}$ & $A 1 / B$ \\
\hline TH & $\begin{array}{l}0.47 \\
92 \%\end{array}$ & $\begin{array}{l}0.82^{\circ} \\
94 \%\end{array}$ & $\begin{array}{l}1.00 \% \\
100 \%\end{array}$ & $\begin{array}{l}1.00^{*} \\
100 \%\end{array}$ & \multirow{3}{*}{$\begin{array}{c}0.88^{*} \\
96 \%\end{array}$} & \multirow{3}{*}{$\begin{array}{l}0.32 \\
88 \%\end{array}$} & $\begin{array}{l}0.78 \% \\
96 \%\end{array}$ & $\begin{array}{l}1.00 \\
100 \%\end{array}$ & $\begin{array}{c}0.67^{\circ} \\
96 \%\end{array}$ & $\begin{array}{l}000 \\
81 \%\end{array}$ \\
\hline IF & $\begin{array}{l}1.00 \% \\
100 \%\end{array}$ & $\begin{array}{l}0.78^{*} \\
94 \%\end{array}$ & $\begin{array}{l}1.00^{\circ} \\
100 \%\end{array}$ & $\begin{array}{l}1.00^{\circ} \\
100 \%\end{array}$ & & & $\begin{array}{l}1.00^{*} \\
100 \%\end{array}$ & $\begin{array}{l}0.63^{*} \\
94 \%\end{array}$ & $\begin{array}{l}000 \\
88 \%\end{array}$ & $\begin{array}{l}000 \\
88 \%\end{array}$ \\
\hline LF & $\begin{array}{c}0.79^{*} \\
96 \%\end{array}$ & $\begin{array}{l}0.78^{\circ} \\
94 \%\end{array}$ & $\begin{array}{l}1.00^{\circ} \\
100 \%\end{array}$ & $\begin{array}{l}0.65^{\circ} \\
94 \%\end{array}$ & & & $\begin{array}{l}037 \\
88 \%\end{array}$ & $\begin{array}{l}063^{\circ} \\
94 \%\end{array}$ & $\begin{array}{r}067^{\circ} \\
94 \%\end{array}$ & $\begin{array}{l}000 \\
88 \%\end{array}$ \\
\hline
\end{tabular}

Table 21. Reliability of the non-dominant/affected hand as measured by the Kappa coefficient

KEY:

Kappa

:Kappa coefficient.

*

:significant Kappa correlation coefficient $(\geq 060)$

$\%$ agree.

PRESS.SENS. :pressure sensitivity

M2PD THRESH. :moving two-point discrimination threshold

STEREO.SCORES :stereognosis scores

PROP.SCORES :proprioception scores

DIR. SCORES :directionality scores

A $1 /$ A2

:Test-retest reliability measured on scores

obtained by Therapıst $A$ on 2 separate testing

occasions (A1, A2)

A $1 / B$

:Inter rater reliability measured on scores obtaıned by Therapist $A(A 1)$ and Therapist $B$

TH

IF

:palmar aspect of the distal phalanx of the thumb

:palmar aspect of the distal phalanx of the index

finger

LF :palmar aspect of the distal phalanx of the little finger

A blank space signifies that both raters scored all children on that variable as normal 


\begin{tabular}{|c|c|c|c|c|c|c|c|c|c|c|}
\hline \multirow{2}{*}{$\begin{array}{c}\text { KAPPA } \\
\& \\
\% \\
\text { AGREE. }\end{array}$} & \multicolumn{2}{|c|}{$\begin{array}{l}\text { PRESS. } \\
\text { SENS. }\end{array}$} & \multicolumn{2}{|c|}{$\begin{array}{c}\text { M2PD } \\
\text { THRESH. }\end{array}$} & \multicolumn{2}{|c|}{$\begin{array}{l}\text { STEREO. } \\
\text { SCORES }\end{array}$} & \multicolumn{2}{|c|}{$\begin{array}{l}\text { PROP. } \\
\text { SCORES }\end{array}$} & \multicolumn{2}{|c|}{$\begin{array}{l}\text { DIR. } \\
\text { SCORES }\end{array}$} \\
\hline & $\mathrm{A} 1 / \mathrm{A} 2$ & $A 1 / B$ & $\overline{A 1 / A 2}$ & $A 1 / B$ & $\overline{A 1 / A 2}$ & $\overline{A 1 / B}$ & $\overline{A 1 / A 2}$ & $A 1 / B$ & $\overline{A 1 / A 2}$ & $A 1 / B$ \\
\hline TH & $\begin{array}{c}0.67^{*} \\
96 \%\end{array}$ & $\begin{array}{l}0.48 \\
88 \%\end{array}$ & $100 \%$ & $100 \%$ & & & $\begin{array}{c}0.64^{*} \\
96 \%\end{array}$ & $\begin{array}{l}0.63^{\circ} \\
94 \%\end{array}$ & $\begin{array}{l}1.00^{\circ} \\
100 \%\end{array}$ & $\begin{array}{c}0.00 \\
100 \%\end{array}$ \\
\hline IF & $\begin{array}{l}0.37 \\
88 \%\end{array}$ & $\begin{array}{l}0.60^{\circ} \\
88 \%\end{array}$ & $100 \%$ & $100 \%$ & $\begin{array}{l}0.64^{*} \\
96 \%\end{array}$ & $\begin{array}{l}0.48 \\
75 \%\end{array}$ & $\begin{array}{c}0.64^{\circ} \\
96 \%\end{array}$ & $\begin{array}{l}0.63^{\circ} \\
94 \%\end{array}$ & $100 \%$ & $100 \%$ \\
\hline LF & $\begin{array}{l}0.37 \\
88 \%\end{array}$ & $\begin{array}{l}0.46 \\
88 \%\end{array}$ & $\begin{array}{l}0.00 \\
92 \%\end{array}$ & $\begin{array}{l}0.65^{*} \\
94 \%\end{array}$ & & & $\begin{array}{c}0.64^{\circ} \\
96 \%\end{array}$ & $\begin{array}{l}0.00 \\
94 \%\end{array}$ & $\begin{array}{l}1.00^{\circ} \\
100 \%\end{array}$ & $\begin{array}{c}0.00 \\
100 \%\end{array}$ \\
\hline
\end{tabular}

Table 22: Reliability of the dominant/'non-affected' hand as measured by the Kappa coefficient

\section{KEY:}

Kappa

$\%$ agree.

PRESS. SENS. M2PD THRESH.

STEREO. SCORES PROP.SCORES DIR. SCORES
A $1 / A 2$

A1/B

TH

IF

LF
:Kappa coefficient.

:significant Kappa correlation coefficient $(\geq 0.60)$ :this is actual percentage agreement between the 2 scores obtained on 2 separate testing occasions :pressure sensitivity :moving two-point discrimination threshold :stereognosis scores :proprioception scores :directionality scores

:Test-retest reliability measured on scores obtained by Therapist $A$ on 2 separate testing occasions (A1, A2)

:Inter rater reliability measured on scores obtained by Therapist $A(A 1)$ and Therapist $B$ :palmar aspect of the distal phalanx of the thumb :palmar aspect of the distal phalanx of the index finger

:palmar aspect of the distal phalanx of the little finger

A blank space signifies that both raters scoied all children on that variable as normal 


\begin{tabular}{|c|c|c|c|c|c|c|c|c|c|c|}
\hline \multirow[t]{2}{*}{ I.C.C. } & \multicolumn{2}{|c|}{$\begin{array}{l}\text { PRESS. } \\
\text { SENS. }\end{array}$} & \multicolumn{2}{|c|}{$\begin{array}{c}\text { M2PD } \\
\text { THRESH. }\end{array}$} & \multicolumn{2}{|c|}{$\begin{array}{l}\text { STEREO. } \\
\text { SCORES }\end{array}$} & \multicolumn{2}{|c|}{$\begin{array}{l}\text { PROP. } \\
\text { SCORES }\end{array}$} & \multicolumn{2}{|c|}{$\begin{array}{c}\text { DIR. } \\
\text { SCORES }\end{array}$} \\
\hline & $A 1 / A 2$ & $A 1 / B$ & $\overline{A 1 / A 2}$ & $A 1 / B$ & $A 1 / A 2$ & $\overline{A 1 / B}$ & $\overline{A 1 / A 2}$ & $A 1 / B$ & $\overline{A 1 / A 2}$ & $\overline{A 1 / B}$ \\
\hline TH & $0.82^{\circ}$ & $0.88^{\circ}$ & 0.40 & $0.96^{*}$ & & & 0.00 & $0.89^{*}$ & $0.68^{*}$ & 0.22 \\
\hline IF & $0.81^{\circ}$ & $0.87^{\circ}$ & $0.69^{\circ}$ & $0.95^{\circ}$ & $0.87^{*}$ & $0.74^{*}$ & 0.00 & 0.00 & 0.30 & $0.71^{\circ}$ \\
\hline LF & $0.66^{\circ}$ & $0.88^{\circ}$ & $0.71^{\circ}$ & $0.66^{\circ}$ & & & 0.01 & 0.00 & 0.20 & 0.13 \\
\hline
\end{tabular}

Table 23: Reliability using the intraclass correlation coefficient on the non-dominant/affected hand.

\begin{tabular}{|c|c|c|c|c|c|c|c|c|c|c|}
\hline \multirow{2}{*}{ I.C.C. } & \multicolumn{2}{|c|}{$\begin{array}{c}\text { PRESS. } \\
\text { SENS. }\end{array}$} & \multicolumn{2}{c|}{$\begin{array}{c}\text { M2PD } \\
\text { THRESH. }\end{array}$} & \multicolumn{2}{c|}{$\begin{array}{c}\text { STEREO. } \\
\text { SCORES }\end{array}$} & \multicolumn{2}{c|}{$\begin{array}{c}\text { PROP. } \\
\text { SCORES }\end{array}$} & \multicolumn{2}{c|}{$\begin{array}{c}\text { DIR. } \\
\text { SCORES }\end{array}$} \\
\cline { 2 - 8 } & A1/A2 & A1/B & A1/A2 & A1/B & A1/A2 & A1/B & A1/A2 & A1/B & A1/A2 & A1/B \\
\hline TH & $0.73^{*}$ & $0.83^{*}$ & 0.12 & 0.34 & & & 0.00 & 0.00 & 0.00 & 0.00 \\
\hline IF & 0.62 & $0.84^{*}$ & 0.40 & 0.31 & 0.12 & 0.33 & 0.01 & 0.00 & 0.22 & 0.22 \\
\hline LF & 0.43 & $0.78^{*}$ & 0.27 & $0.66^{*}$ & & & 0.00 & 0.00 & 0.00 & 0.10 \\
\hline
\end{tabular}

Table 24: Reliability using the intraclass correlation coefficient on the dominant/'non-affected' hand.

KEY:

I.C.C. :Intraclass correlation coefficient.

*

:significant ICC $(\geq 0.65)$

PRESS. SENS. :pressure sensitivity threshold

M2pd THRESH. :moving two-point discrimination threshold

STEREO SCORES :stereognosis scores

PROP.SCORES :proprioception scores

DIR.SCORES :directionality scores

A $1 /$ A2

:Test-retest reliability measured on scores

obtained by Therapist $A$ on 2 separate testing

occasions (A1, A2)

A1/B :Inter rater reliability measured on scores obtained

by Therapist $A$ (A1) and Therapist $B$

TH

:palmar aspect of the distal phalanx of the thumb

IF

:palmar aspect of the distal phalanx of the index

finger

LF

:palmar aspect of the distal phalanx of the little finger 


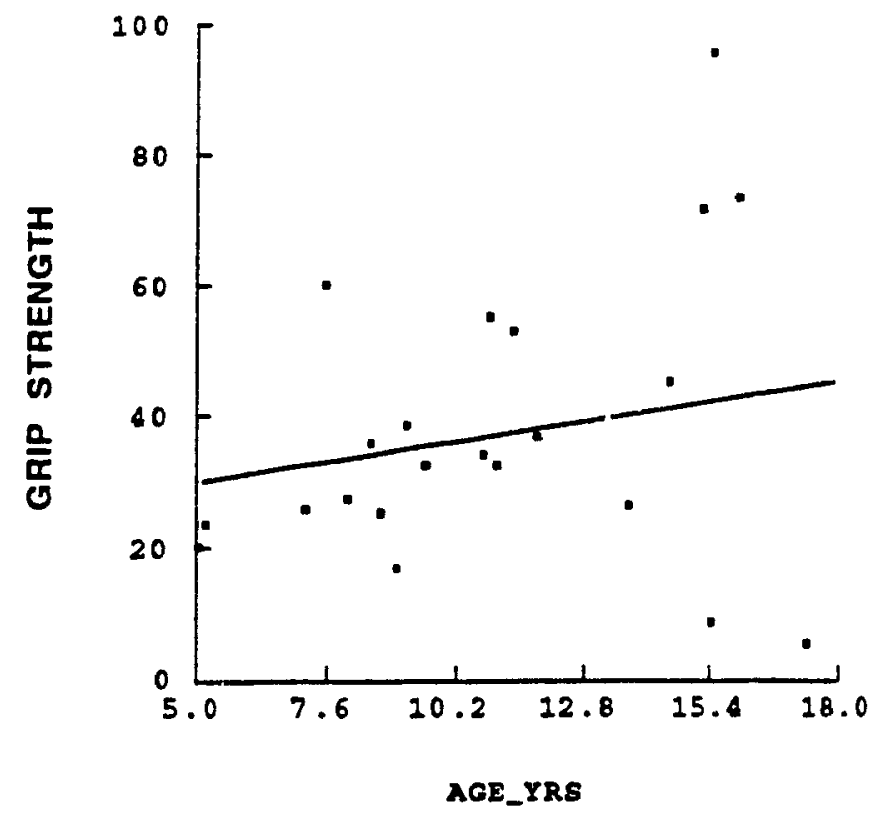

Figure 1:Scatter diagram of control values of the grip strength of the non-dominant hand plotted as a function of age.

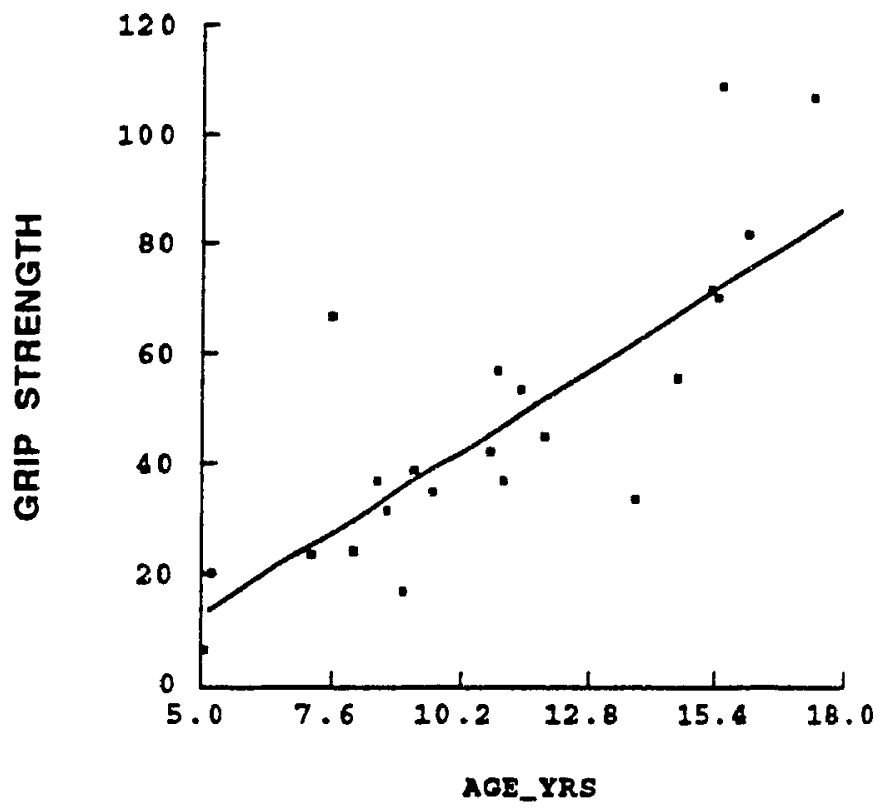

Figure 2: Scatter diagram of control values of the grip strength of the dominant hand plotted as a function of age. 


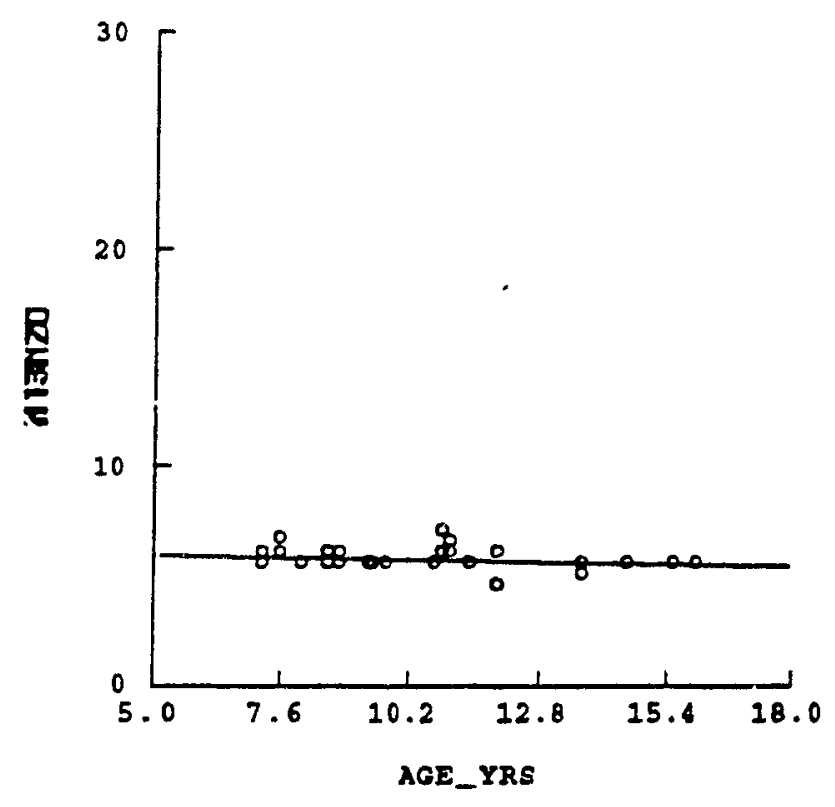

Figure 3: Scatter diagram of control values of N13-N2O interwave latencies plotted as a function of age. 

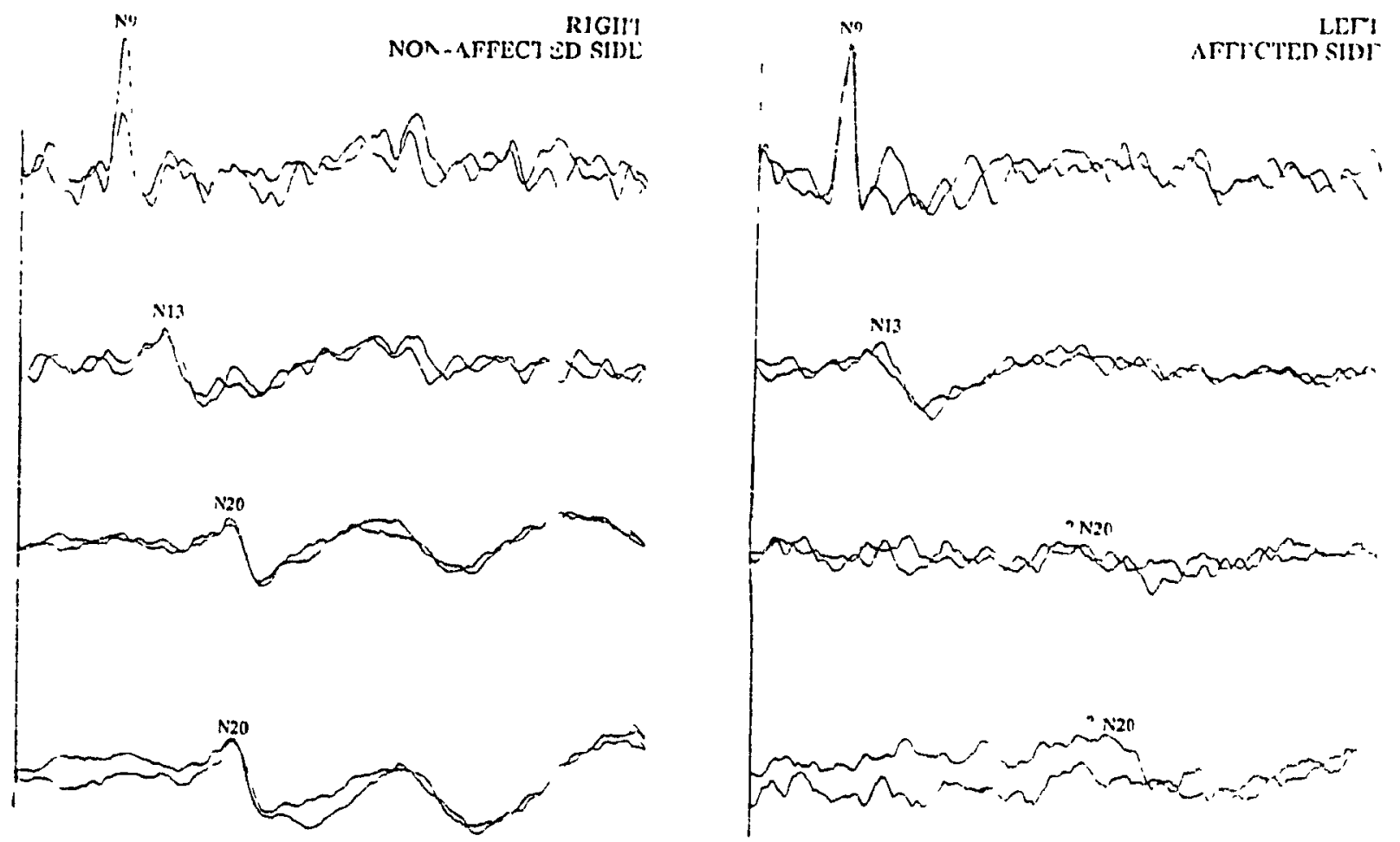

Figure 4: SEP waveform recordings of a child with left hemiplegia. 


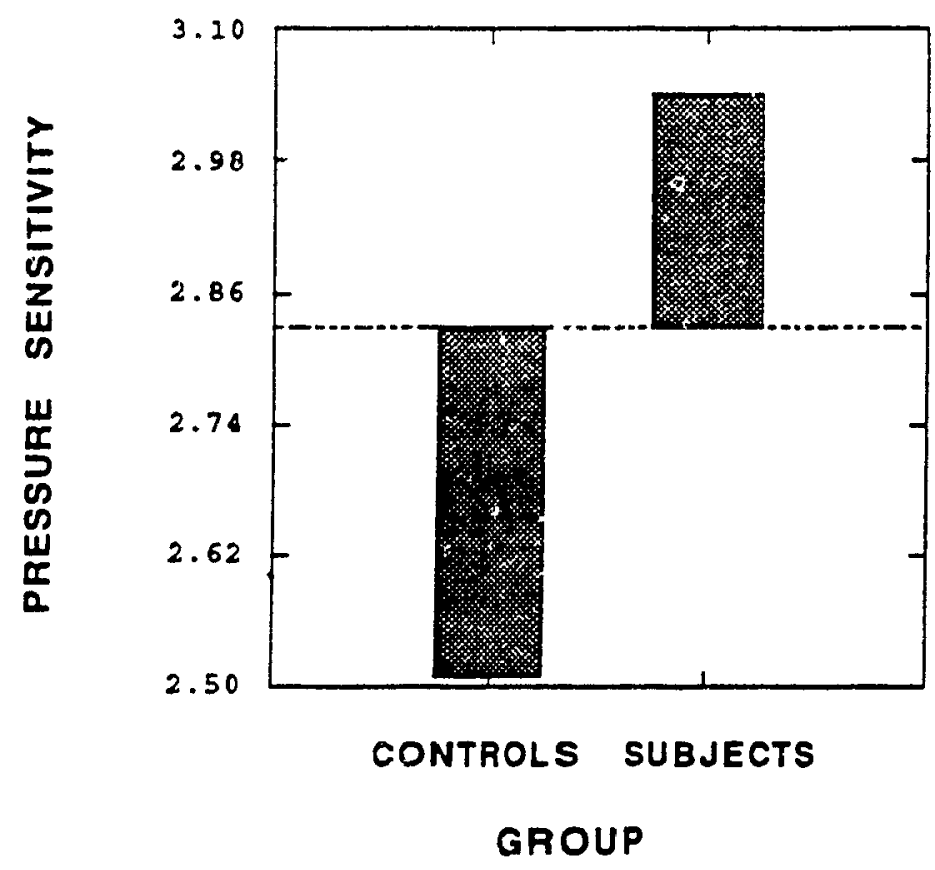

Figure 5: Mean pressure sensitivity thresholds of the thumb on the non-dominant/affected hand. 


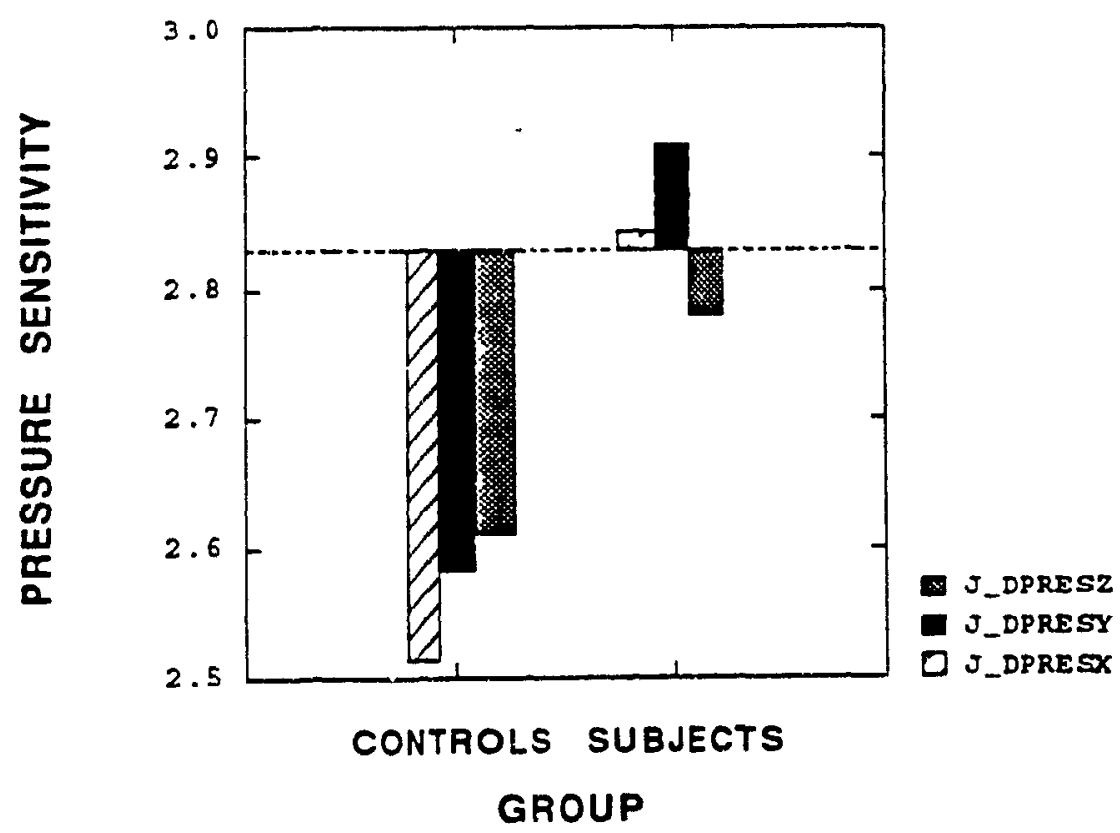

Figure 6: Mean pressure sensitivity thresholds of the dominant/'nonaffected' hand.

KEY:

J_DPRESX :Mean pressure sensitivity thresholds of the thumb of the dominant/'non-affected' hand

J_DPRESY :Mean pressure sensitivity thresholds of the index finger of the dominant hand

J_DPRESZ :Mean pressure sensitivity thresholds of the little finger of the dominant hand 


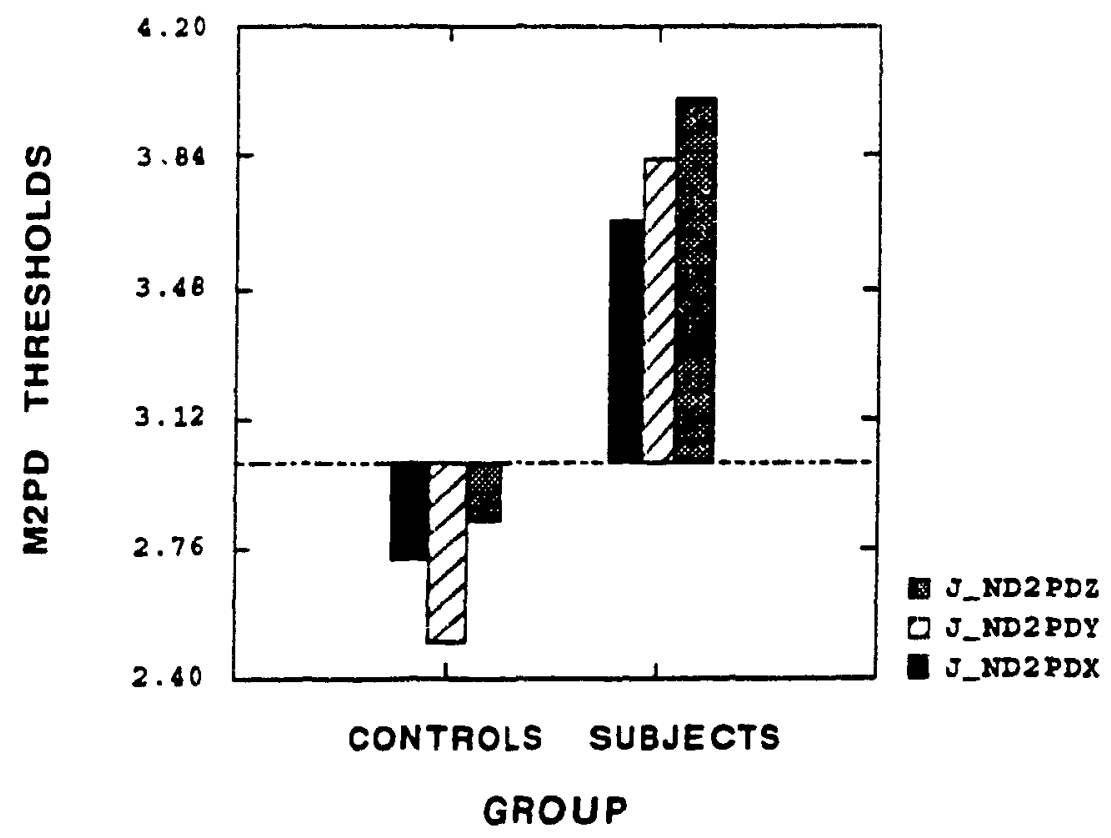

Figure 7: Mean m2pd thresholds of the non-dominantaffected hand.

KEY:

J_ND2PDX :Mean moving 2-point discrimination (m2pd) thresholds of the thumb of the non-dominant/affected hand

J_ND2PDY :Mean m2pd thresholds of the index finger of the nondominant hand

J_ND2PDZ :Mean m2pd thresholds of the little finger of the nondominant hand 


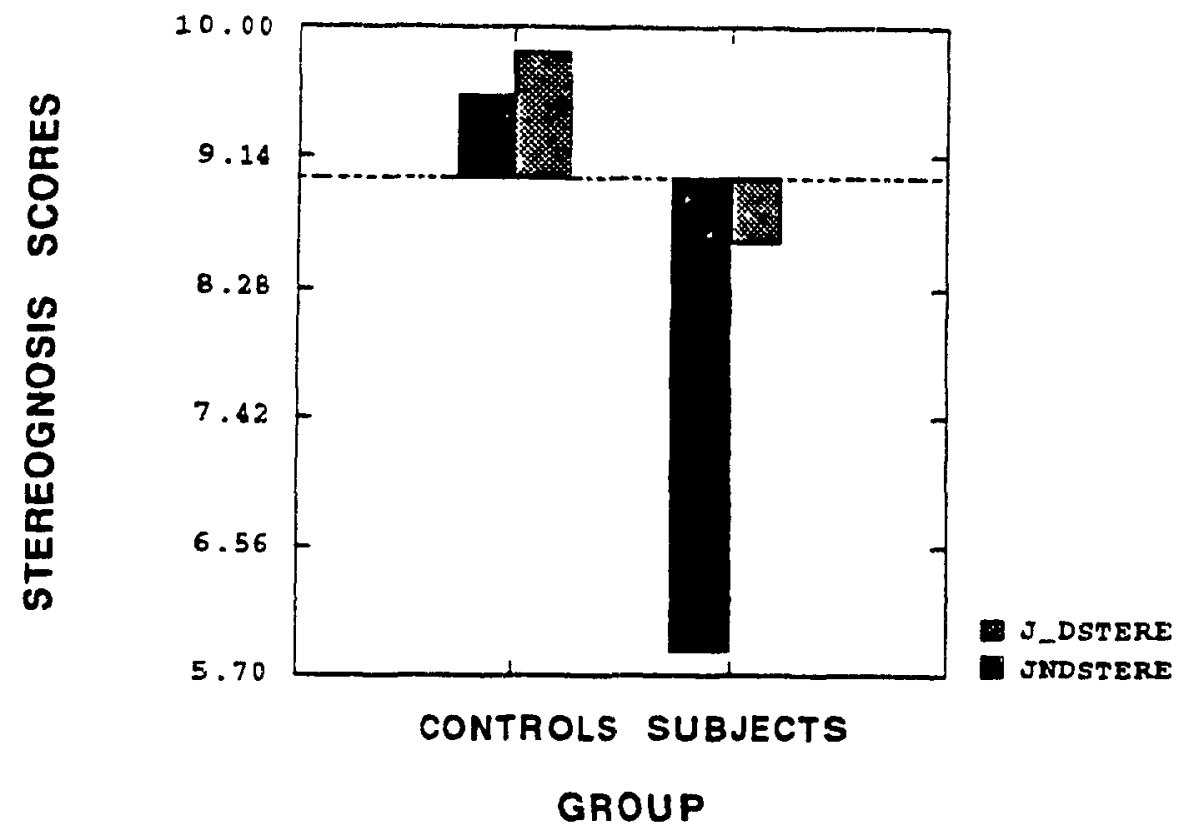

Figure 8: Mean stereognosis scores of the non-dominant laffected and dominant/'non-affected' hands.

KEY:

J_DSTERE :Mean stereognosis scores of the domınant/'non-affected' hand

JNDSTERE :Mean stereognosis score of the non-domınant/affected hand 


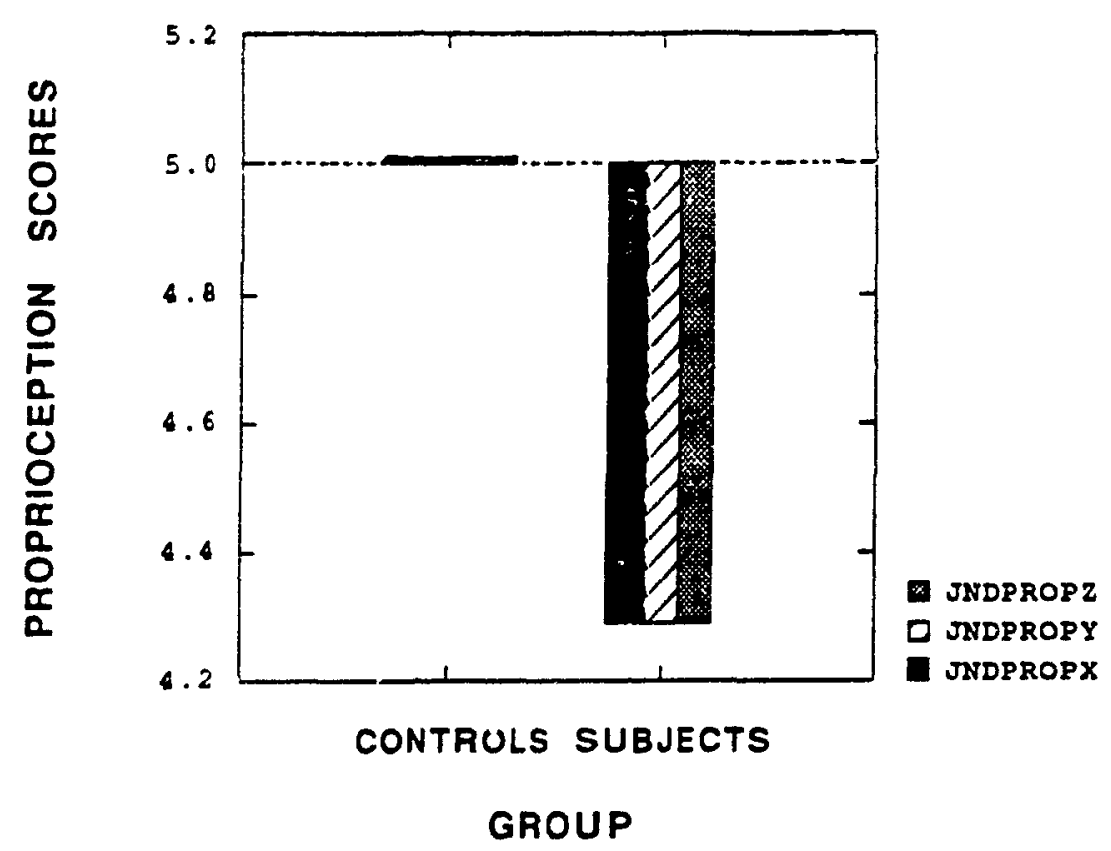

Figure 9: Mean proprioception scores of the non-dominant/affected hand.

KEY:

JNDPROPX :Mean proprioception scores of the non-dominant/affected hand

JNDPROPY :Mean proprioception scores of the non-dominant hand JNDPROPZ :Mean proprioception scores of the non-dominant hand 


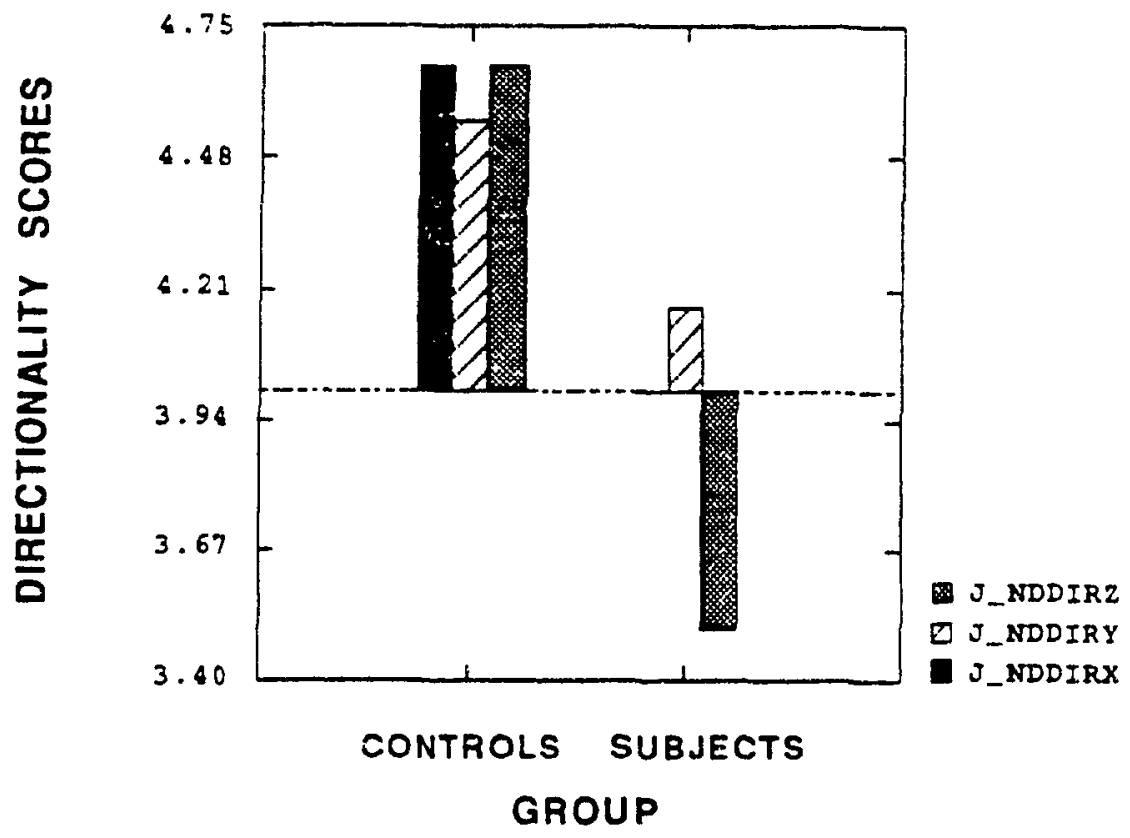

Figure 10: Mean directionality scores of the non-dominant/affected hand.

KEY:

J_NDDIRX :Mean directionality scores of the non-dominant/affected hand

J_NDDIRY :Mean directionality scores of the non-dominant hand

J_NDDIRZ :Mean directionality scores of the non-dominant hand 


\section{APPENDIX A}

\section{CLINICAL DESCRIPTION OF CEREBRAL PALSY (CP)}

1.Spastic CP: Spasticity of the clasp knife type, persistent primitive reflexes, absent or reduced righting and equilibrium reactions, increased tendon jerks and deformities such as kyphosis, lordosis, heel cord shortening and flexion contractures of the elbow, fingers, hip and knee are some of the common clinical symptoms seen. Spastic CP occurs in $60 \%$ of the CP population.

2. Athetoid CP: Here the muscie tone varies from low to high. There is inability to co-contract the muscles around a joint and stabilize it. This leads to joint subluxation. Slow, writhing involuntary movements are present, more distally than proximally. Muscle twitches may also be present. Five percent of the CP population exhibits athetosis.

3. Ataxic CP: Low tone is characteristic of this type of CP A stable base of support is lacking, and patients appear uncoordinated and clumsy. They have poorly timed motor patterns and also have difficulty in maintaining balance. Fourteen percent of the CP population is ataxic.

4. Flaccid CP: Decreased muscie tone is present. This may cause problems in respiratory function. There is a lack of development of stability patterns for gross motor function. Mental retardation is often present in this type of CP

Very often a mixed presentation of $C P$ is seen. These children present with symptoms from more than 1 of the individual types described above (Boone, 1978). 
APPENDIX B

\section{QUESTIONNAIRE TO BE FILLED OUT BY THERAPIST 'A' FOR SUBJECTS}

Name of child:

Age:

Diagnosis:
Sex:

Birthdate

PLEASE CHECK OFF OR FILL IN THE APPROPRIATE SPACE.

1.) Dominance:

Writing

Throwing

Drawing

Using scissors

Using a toothbrush
-Score $1-2-3-4-5$

-Score $1-2-3-4-5$

-Score $1-2-3-4-5$

-Score $1-2-3-4-5$

-Score $1-2-3-4-5$

Scoring:

1 - if the right hand is strongly preferred

2 - if the right is preferred

3 - if indifferent

4 - if the left hand is preferred

5 - if the left hand is strongly preferred

2.) Any other major medical illness present (1.e diabetes,myopathies, neuropathies, peripheral nerve injuries...)

YES

NO

Comments

3.) Does the child have the following Language skills?

A. Expressive Component: (24-27 months).

-Usually uses 2-word or 3-word sentences.

-Often uses personal pronouns correctly ( $I$, you, he, it, me etc.) 
-Asks for help with some personal needs (such as washing hands, going to the toilet, etc.)

YES

NO

B. Receptive Component: (30-36 months).

-Demonstrates an understanding of all common verbs.

-Understands very long and complex sentences.

-Demonstrates an understanding of most common adjectives (i.e. big, small, far, near, tall, short, etc.).

YES

NO

(Bzoch-League Receptive-Expressive Language Scale).

Comments

4.) Does the child have any known visual or hearing impairments?

YES

NO

Comments

5.) Is the child anglophone

francophone

bilingual

other

Comments

6.) Are you aware of any specific sensory deficits in this child? 
YES

NO

Comments 


\author{
APPENDIX C
}

\title{
BZOCH-LEAGUE RECEPTIVE-EXPRESSIVE EMERGENT LANGUAGE SCALE
}

(Bzoch and League, 1970).

RECEPTIVE LANGUAGE (30-33 months):

-demonstrates an understanding of all common verbs -understands very long and complex sentences -demonstrates an understanding of most common adjectives

EXPRESSIVE LANGUAGE (24-27 months):

-usually uses 2-word or 3-word sentences -often uses personal pronouns correctly ( $I$, you, he, it, me, etc.) -asks for help with some personal meeds (such as washing hands, going to the toilet, etc.) 
APPENDIX D

DEPARTMENTS OF NEUROLOGY \& OCCUPATIONAL THERAPY

THE MONTREAL CHILDREN'S HOSPITAL

$\underline{\&}$

SCHOOL OF PHYSICAL \& OCCUPATIONAL THERAPY

MCGILL UNIVERSITY.

THE IMPORTANCE OF SENSORY TESTING

FOR THE MANAGEMENT OF CEREBRAL PALSY

\section{Informed Consent Form}

This is a research study being performed by Jasmıne Cooper under the supervision of Prof. A. Majnemer (School of Physical and Occupational Therapy, McGill (Iniversity) and Dr. B. Rosenblatt (Dept. of Neurology, Montreal Children's Hospital).

Assessments of Cerebral Palsy focus on motor function and developmental abilities whereas sensory function is often less emphasized. However, sensory deficits will influence motor performance. It is hoped that this study will vield information which will help determine the value of performing a standardized sensory assessment in children with cerebral palsy.

The study will involve 2 types of testing procedures Each testing procedures will last for approx. 30 minutes each. The first testing procedure involves performance on a test of hand function and sensation. The test will require manipulation and identification of objects and discrimination of various sensations. During the testing, the hand will sometimes be placed behind a screen and hidden from view. This test will be repeated again on the same day and at a later date, so that the reliability of the test can be evaluated.

The second testing procedure involves the recording of Somatosensory Evoked Potentials (SEP). The SEP evaluates the brain's response to stimulation at the wrist. These procedures are in no way painful or dangerous to the child and no needles are used. Recording devices will be pasted to the head and neck. A sensory 
stimulator is placed on the wrist for this test. The child will be examined by a paediatric neurologist as well.

All personal data will be kept confidential. Any pertinent findings will be given to the referring therapist/doctor. Participation in this research is strictly voluntary, and your child/ward may withdraw at any time without prejudice.

Thanking you for your cooperation. If you have any questions, please feel free to contact Jasmine Cooper or Prof. Annette Majnemer at The Montreal Children's Hospital, Room 508, A Wing. Tel:934 4400/Ext. 2902 or 4453.

Jasmine Cooper, B.Sc.

Graduate Student

Rehabilitation Science

School of Physical \&

Occupational Therapy

McGill University

Annette Majnemer, Ph.D.

Associate Professor

School of Physical \&

Occupational Therapy

McGill University.
Bernard Rosenblatt, M.D.C.M., F.R.CP (C) Department of Neurology Montreal Children's Hospital 
I have been informed of the procedures to be used and understand them. I consent to the participation of my child/ward in the research study. I also understand that participation will not interfere in any way with my child's ongoing care and that I am free to withdraw from the study at any time.

Signature of the Parent/Guardian:

Signature of patient:

Signature of witness:

Signature of Principal investigator: 


\section{APPENDIXE}

\section{QUESTIONNAIRE TO BE FILLED OUT BY THERAPIST 'A' FOR CONTROLS}

Name of child:

Age:

Name of school:
Sex:

Birthdate:

PLEASE CHECK OFF OR FILL IN THE APPROPRIATE SPACE.

1.) Dominance:

$\begin{array}{ll}\text { Writing } & \text {-Score } 1-2-3-4-5 \\ \text { Throwing } & \text {-Score } 1-2-3-4-5 \\ \text { Drawing } & \text {-Score } 1-2-3-4-5 \\ \text { Using scissors } & \text {-Score } 1-2-3-4-5 \\ \text { Using a toothbrush } & \text {-Score } 1-2-3-4-5\end{array}$

Scoring:

1 - if the right hand is strongly preferred

2 - if the right is preferred

3 - if indifferent

4 - if the left hand is preferred

5 - if the left hand is strongly preferred

2.) Does the child have any known medical illness (i.e. diabetes, orthopaedic or nerve injuries of the hand)?

YES;

No

Comments:

3.) Does the child have the following Language skills?

A. Expressive Component: (24-27 months).

-Usually uses 2-word or 3-word sentences. 
- Often uses personal pronouns correctly ( 1 , you, he, it, me etc.) -Asks for help with some personal needs (such as washing hands, going to the toilet, etc.)

YES

NO

B. Receptive Component: (30-36 months).

-Demonstrates an understanding of all common verbs.

-Understands very long and complex sentences.

-Demonstrates an understanding of most common adjectives

(i.e. big, small, far, near, tall, short etc.).

YES

NO

(Bzoch-League Receptive-Expressive Language Scale).

Comments:

4.) Does the child have any known visual or hearing impairments?

YES

NO

Comments:

5.) Is the child anglophone

francophone

bilingual

other

Comments: 


\section{APPENDIX F}

\section{DOMINANCE OF THE HAND}

(adapted from the Crovitz-Zener and Oldfield Questionnaires)

(Bryden, 1977).

\section{WRITING}

THROWING

DRAWING

CUTTING WITH SCISSORS

\section{USING A TOOTHBRUSH}

Scoring:

1 - if the right hand is strongly preferred

2 - if the right hand is preferred

3 - indifferent

4 - if the left hand is preferred

5 - If the left hand is strongly preferred 


\section{APPENDIX G \\ SENSORY - MOTOR ASSESSMENT BATTERY}

NAME:

DATE OF BIRTH:

AGE:

SEX:

DATE OF EVALUATION:

EVALUATOR:

\section{MOTOR ASSESSMENT.}

1. Passive Range of Motion:

ELBOW

Extension to Flexion $0-145^{\circ}$

RADIOULNAR JOINTS

Pronation

$0-90^{\circ}$

Supination

$0-80^{\circ}$

WRIST

Flexion

$0-80^{\circ}$

Extension

$0.70^{\circ}$

THUMB

MP Extension to Flexion $0-50^{\circ}$

INDEX FINGER

MP Flexion

$0-90^{\circ}$

MP Hyperextension 
LITTLE FINGER

MP Flexion

$0-90^{\circ}$

MP Hyperextension

$0.45^{\circ}$

2. Muscle Tone of Upper Extremities:

$$
\begin{aligned}
& 0 \text { - hypotonic } \\
& 1 \text { - slightly floppy } \\
& 2 \text { - normal } \\
& 3 \text { - moderately hypertonic } \\
& 4 \text { - ophistotonic } \\
& 5 \text { - mixed tonicity }
\end{aligned}
$$

\section{LEFI}

3. Functional Evaluation of the Congenitally Anomalous Hand:

$$
\begin{aligned}
& 0 \text { - no grasp possible } \\
& 1 \text { - inappropriate grasp present } \\
& 2 \text { - normal grasp present }
\end{aligned}
$$

Hook

Lateral

Power

Palmar 
Grip strength(lbs.)

$\begin{array}{lll}\text { Mean: } & & \\ \text { Pinch Strength (lbs.) } & & \\ & \text { Mean: } & \text { BEFT }\end{array}$

\section{SENSORY ASSESSMENT.}

1. Light touch-deep pressure (Semmes-Weinstein monofilarrents):

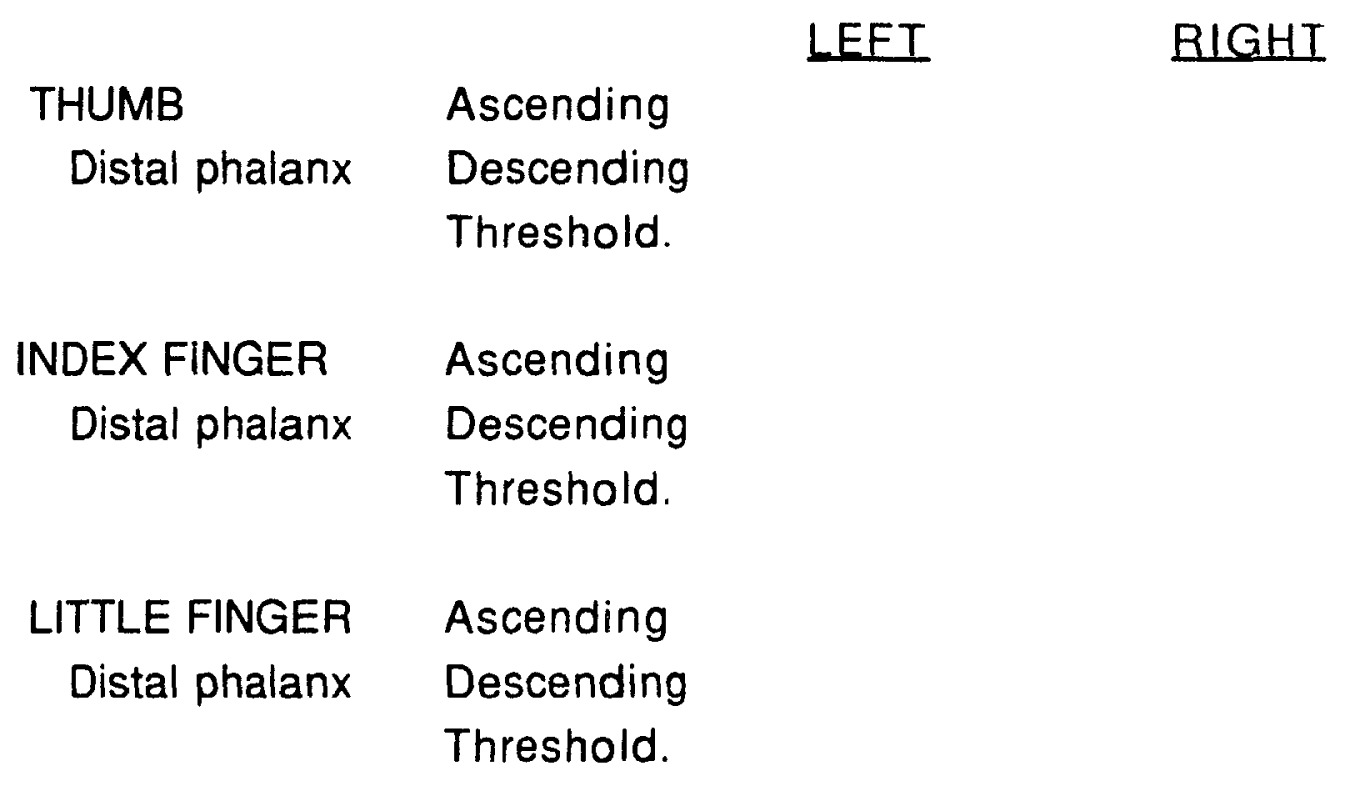

2. Moving two-point discrimination (Disk-criminator):

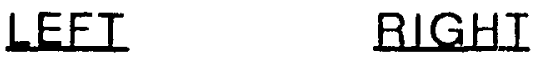

THUMB (mm)

Distal phalanx 
INDEX FINGER (mm)

Distal phalanx

LITTLE FINGER $(\mathrm{mm})$

Distal phalanx

3. Stereognosis:

LEFI

Circle

Square

Diamond

Triangle

Octagon

Toothbrush

Candy

Comb

Cup

Ball

Score(10):

4. Proprioception:

\section{LEFI}

THUMB

$M P$ joint

Score (5):

INDEX FINGER

MP joint

Score (5): 



\section{APPENDIXH}

\section{EINSTEIN NEONATAL NEUROBEHAVIOURAL ASSESSMENT SCALE} (Kurtzberg et al, 1979).

\section{TONUS}

0 - hypotonic (little or no resistance to manipulation)

1 - slightly floppy

2 - normal

3 - moderately hypertonic (exaggerated to extension of extremities, tendency for head extension to predominate)

4 - ophistotonic

5 - mixed tonicity across head, trunk, upper and lower extremities 


\title{
APPENDIX I
}

\section{FUNCTIONAL EVALUATION OF THE CONGENITALLY ANOMALOUS} HAND

(Weiss and Flatt, 1971).

\author{
Hook Grip - hold a bag. \\ Power Grip - hammer pegs into a pegboard. \\ Lateral Pinch - hold a key. \\ Palmar Pinch - String a bead.
}

Scoring:

0 - no handling of the object present

1 - object manipulation present with inappropriate grasp

2 - normal grip/grasp present 
APPENDIX J

3

NEUROLOGIC EXAMINATION 
DATE:

Referring $D r$.

No.

AREA OF CONCERN:

FAMIIY HISTORY

PRENATAI

IABOUR

DEIIVERY

NEONATAI

EDG

GSTN

B.WT.

HOSPITAL

PREVIOUS ADMISSIONS:

DEVELOPMENT
smile
sat
crawl
stood
cruise
step
word
phrase
toilet trained
pedal trike
tic shoe lace

Head injury

Unconse

Soizures

Faints

Moticn Sick

RAP

H.A

Eating

Sleeping

Night Terrors

Enuresis

Medications

Allergies 
MI:ZGi:"

UEI GIT

IIEND CIWS

GEUERIL
IEIraL STATLS

SPEECH

SPINE

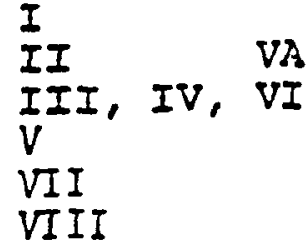

MOTOR BuzK

Tone

Power

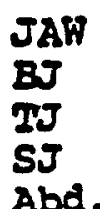

Abd. Up

Iow

ADD

KJ

AJ

PIANTAR

FUNDI

R.

I

PIELDS

$X I$

XII

GAIT On Toes
On Heels

RAM UE

$F-\mathbb{N}$

$\mathrm{H}-\mathrm{K}$
TANDEY POIWard

Baclewards

STAND

EOP

JORLP $\begin{array}{ll}\text { SENSORY Touch } & \begin{array}{l}\text { Ton } \\ \text { Pin }\end{array}\end{array}$

DIAGNOSIS :
Jt.Position

Vibration 2pt

stereognosis Graphesthesia

PIAN: 


\section{BIBLIOGRAPHY}

Ager,C.L. Olivett,B.L. and Johnson,C.L. Grasp and strength in children 5 to 12 years old (hand assessment, hand function, hand rehabilitation). Am.J.Occup. Ther 38:107-113,1984.

Asanuma, H. and Arissian,K. Motor deficit following interruption of sensory inputs to the motor cortex of the monkey. Kyete Symoosia(EEG Supplement no.36). Edited by Buser,P.A., Cobb,W.A. and Okuma,T., Amsterdam: Elsevier Biomedical Press, 1982,pp.415-421.

Asanuma,H. and Arissian,K. Experiments on functional role of peripheral input to motor cortex during voluntary movements in the monkey. J.Neurophysiol 52:212-227,1984.

Ayres,J.A. Southern California Sensory Integration Tests (SCSIT). California:Western Psychological Services, Copyright 1980.

Bach-y-Rita,P. Central nervous system lesions:Sprouting and unmasking in rehabilitation. Arch.Phys.Med.Rehabil. 62:413417,1981 .

Banus,B.S. Treatment and management In:'The Developmental Therapist' a prototype of the Pediatric Occupational Therapist. Edited by Banus,B.S. Kent,C.A. de Sinson Norton,Y. Sukiennicki,D.A. and Becker,M.L. 2nd edition, New Jersey:Slack Incorporated, 1979,pp.201-236.

Bartel,P. Conradie,J. Robinson,E. Prinsloo,J. Becker,P. The relationship between median nerve somatosensory evoked potential latencies and age and growth parameters in young children. Electroencephalogl. Clin.Neurophysiol. 68:180-186,1987.

Bell,J.A. Sensibility testing:state of the art. In:Rehabilitation of the hand. Edited by Hunter,J.M., Schneider,LH, Mackin,E.J. and 
Callahan,A.D. 2nd edition, St. Louis:The C.V.Mosby Co., 1984,pp.390398.

Bolanos,A.A. Bleck,E.E. Firestone,P. and Young,L. Comparison of stereognosis and two-point discrimination testing of the hands of children with cerebral palsy. Dev.Med.Child Neurol. 31:371-376,1989.

Boone,D.R. Cerebral Palsy: Bobbs Merrill studies in communicative disorders Edited by Halpern,H. Indianopolis:Bobbs-Merrill Educational Publishing, 1978,pp.4-8.

Brown,J.K. Hemiplegia in children. In:Textbook of Pediatrics. 3rd edition. Edited by Forfar,J.O. and Arneil,G., Edinburgh:Churchill Livingstone, 1984, pp.707-715.

Brown,J.K. van Rensburg,F. Walsh,G. Lakie,M. and Wright,G.W. A neurological study of hand function of hemiplegic children. Dev.Med.Child Neurol, 29:287-304,1987.

Brown,J.V. Schumacher,U. Rohlmann,A. Ettlinger,G. Schmidt,R.C. and Skerczek,W. Aimed movements to visual targets in hemiplegic and normal children:is the 'good' hand of children with infantile hemiplegia also normal? Neuropsychologia. 27:283-302,1989.

Bryden,M.P. Measuring handedness with questionnaires. Neuropsychologia, 15:617-624,1977.

Bzoch,K.R. and League,R. Bzoch-Leaque Receptive-Expressive Emergent Scale (REEL). Copyright 1970.

Callahan,A.D. Sensibility testing:clinical methods. In:Rehabilitation of the hand. Edited by Hunter,J.M., Schneider,LH, Mackin,E.J. and Callahan,A.D. 2nd edition, St.Louis:C.V.Mosby Co., 1984,pp.407-431. 
Carlson, M. Characteristics of sensory deficits following lesions of Broadmann's areas 1 and 2 in the postcentral gyrus of Macaca Mulatta. Brain Res. 204:424-430,1981.

Carmon,A. Disturbances of tactile sensitivity in patients with unilateral cerebral lesions. Cortex. 7:83-97,1971.

Chapman,C.E. and Wiesendanger,M. The physiological and anatomical basis of spasticity: a review. Physiother.Can. 34:125-136,1982.

Chiappa,K.H. (Ed.) Principles of evoked potentials In:Evoked potentials in clinical medicine. 2nd edition, New York:Raven Press, 1990,pp.1-36.

Chiappa,K.H. (Ed.) Short-latency evoked potentials:Methodology In:Evoked potentials in clinical medicine 2nd edition, New York:Raven Press, 1990,pp.307-370.

Chiappa,K.H. (Ed.) Short-latency evoked potentials:Interpretation In:Evoked potentials in clinical medicine 2nd edition, New York:Raven Press, 1990,pp.371-437.

Clark,P.N. and Allen,A.S. Qccupational Therapy for children 1st edition. Toronto:C.V.Mosbj' Co. 1985,pp.321-322.

Cracco,J. Somatosensory evoked potentials in infants and children L.Child Neurol 4:70-72,1989.

Cracco,R.O. Cracco,J.B. and Anziska,B.J. Somatosensory evoked potentials in man:cerebral, subcortical and peripheral nerve potentials. Amer.J.EEG.Technol, 19:59-81,1979.

Corkin,S. Milner,B. and Rasmussen,T. Somatosensory thresholds:Contrasting effects of postcentral gyrus and posterior parietal-lobe excisions. Arch.Neurol, 23:41-58,1970. 
Corkin,S. Milner,B. and Taylor,L. Bilateral sensory loss after unilateral cerebral lesion in man. Irans.Am.Neurol.Assoc. 98:118$122,1973$.

Critchley, $M$. The pher,omenon of tactile inattention with special reference to parietal lesions. Brain 72:538-561,1949.

Curry,J. and Exner,C. Comparison of tactile preferences in children with and without cerebral palsy. Am J.Occup Ther 42:371-377,1988.

Dannenbaum,R.M. and Dykes,R.W. Sensory loss in the hand after sensory stroke:Therapeutic rationale. Arch.Phys.Med.Rehabil. 69:833839,1988 .

Darian-Smith,I. Sugitani,M. and Heywood,J. Touching textured surfaces:cells in somatosensory cortex respond both to finger movement and to surface features. Science, 218:906-909,1982.

DeGangi,G.A. Sensorimotor tests In:A Therapist's Guide to pediatric Assessment Edited by King-Thomas,L. and Hacker,B.J. Toronto:Little, Brown and Company, 1987,pp.143-226.

Dellon,A.L. Touch sensibility in the hand. LHand Surg, 9:11-13,1984.

Dellon,A.L. and Kailman,C.H. Evaluation of functional sensation in the hand. LHand Sure 8:865-870,1983.

Dellon,A.L. Mackinnon,S.E. and Crosby,P.M. Reliability of two-point discrimination measurements. L.Hand Sura 12:693-696,1987.

Deuel,R.K. and Regan,D.J. Parietal hemineglect and motor deficits in the monkey. Neuropsychologia, 23:305-314,1985.

Dykes,R.W. The anatomy and physiology of the somatic sensory cortical regions. Proa.Neurobiol. 10:33-38,1978. 
Dykes,R.W. Parallel processing of cutaneous information in the somatosensory system of the cat. Can.J.Neurol.Sci. 9:9-19,1982

Essick,G.K. and Whitsel,B.L. Assessment of the capacity of human subjects and SI neurons to distinguish opposing directions of stimulus motion across the skin. Brain Res.Rev 10:187-212,1985.

Fagan,E.R., Taylor,M.J. and Logan,W.J. Somatosensory evoked potentials:Part I. A review of neural generators and special considerations in Pediatrics. Pediatr.Neurol. 3:189-196,1987.

Freund,H-J. Differential effects of cortical lesions in humans. Ciba Found.Symp. 132:269-281,1987.

Gelberman,R.H. Szabo,R.M. Williamson,R.V. and Dımick,M.P. Sensıbilıty testing in peripheral compression syndromes:an experimental study in humans. J.Bone Joint Sura. 65:632-638,1983.

Gellis,M. and Pool,R. Two-point discrimination distances in the normal hand and forearm. Plast.Reconstr. Sura. 59:57-63,1977.

Giblin,D. Somatosensory evoked potentials in healthy subjects and in patients with lesions of the nervous system. Am.N.Y.Acad.Sci 112:93-141,1964.

Gilmore,R. The use of somatosensory evoked potentials in infants and children. J.Child Neurol. 4:3-19,1989.

Green,W.T. and Banks,H.H. Flexor carpi ulnaris transplant and its use in cerebral palsy. J.Bone Joint Sura. 44:1343-1352,1962.

Guyton,A.C. (Ed.) Organization of the nervous system. In:Iextbook of Medical Physiology, 8th edition, Montreal:W.B.Saunders Co., $1991, p p .478-494$. 
Guyton,A.C. (Ed.) Somatic sensations I-General organization; the tactile and position senses. In:Iextbook of Medical Physiology 8th edition, Montreal:W.B.Saunders Co., 1991,pp.507-519.

Hacker,B.J. and Porter,P.B. Use of standardized tests with the physically handicapped. In:A Therapist's Guide to pediatric Assessment Edited by King-Thomas,L. and Hacker,B.J. Toronto:Little, Brown and Company, 1987,pp.35-42.

Hill,A. Current concepts of hypoxic-ischemic cerebral injury in the term newborn. Pediatr.Neurol. 5:317-325,1991.

Hohman,L.B. Baker,M.D. and Reed,R. Sensory disturbances in children with infantile hemiplegia, triplegia and quadriplegia. Am.J.Phys.Med. $37: 1-6,1958$.

Hyvarinen,J. and Poranen,A. Function of the parietal association area 7 as revealed from cellular discharges in alert monkeys. Brain. 97:673-692,1974.

Jones,B. The perception of passive joint-movements by cerebralpalsied children. Dev.Med.Child Neurol. 18:25-30,1976.

Jones,L. The assessment of hand function:a critical review of techniques. JHand Surg. 14:221-228,1989.

Jones,M.H. and Ogg,H.L. The use of sensory modalities in the training of infantile cerebral palsied patients. Clin.Orthop, 46:55-81,1966.

Kaas,J.H. What, if anything is SI? Organization of first somatosensory area of cortex. Physiol.Rev. 63:206-231,1983.

Kaas,J.H. Nelson,R.J. Sur,M. Lin,C-S. and Merzenich,M.M. Multiple representations of the body within the primary somatosensory cortex of primates. Science, 204:521-523,1979. 
Kandel,E.R. and Jessel,T.M. Touch. In:Principles of Neural Science Edited by Kandel,E.R., Schwartz,J.H. and Jessel,T.M. 3rd edition,. New York:Elsevier Science Publishing company, 1991,pp.367-384.

Kaplan,M.S. Plasticity after brain lesions:Contemporary concepts. Arch.Phys.Med.Rehabil. 69:984-991,1988.

Karnaze,D. Fisher,M. Ahmadi,J. and Gott,P. Short-latency somatosensory evoked potentials correlate with the severity of the neurological deficit and sensory abnormalities following cerebral ischemia. Electroencephaloar.Clin.Neurophysiol. 67:147-150,1987.

Kenney,W.E. Certain sensory defects in cerebral palsy. Clin.Orthep 27:193-195,1963.

Kenney,W.E. The importance of sensori-perceptuo-gnosia in the examination, the understanding and management of cerebral palsy. Clin.Orthor 46:55-81,1!:66.

Kenney,W.E. Pansera,F.T. and Verry,L.M. Training hands in children with astereognosis due to cerebral palsy. L.Bone Joint Surg. $44: 1490,1962$.

Kurtzberg,D. Vaughan,H.G.Jr. Daum,C. Grellong,B.A. Albin,S. and Rotkin,L. Neurobehavioral performance of low-birth weight infants at 40 weeks conceptional age:comparison with normal full term infants. Dev.Med.Child Néurol, 21:590-607,1979.

Laget,P. Salbreux,R. Raimbault,J. d'Allest,A.M. and Marianı,J. Relationship between changes in somesthetic evoked responses and electroencephalographic findings in the child with hemiplegia. Dev.Med.Child Neurol. 18:620-631,1976.

Larson,S. SancesJr,A. and Baker,J. Evoked cortical potentials in patients with stroke. Circulation. 33(2):15-19,1966. 
Laureau,E. Majnemer,A. Rosenblatt,B. and Riley,P. A longitudinal study of short latency somatosensory evoked responses in healthy newborns and infants. Electroencephalogr. Clin. Neurophysiol. 71:100108,1988 .

Lesny,1. Disturbance of 2-point discrimination sensitivity in different forms of cerebral palsy. DevMed.Child Neurol. 13:330$334,1971$.

Levin,S. Pearsall.G and Ruderman,R.J. Von Frey's method of measuring pressure sensibility in the hand:an engineering analysis of the Weinstein-Semmes pressure aesthesiometer. JHand Sura 3:211216,1978 .

Liberson,W. Study of evoked potentials in aphasics. Am.J.Phys.Med. 45:135-142,1966.

Logan,W. Taylor,M and Voros,J. Evoked potentials in non progressive disorders of the develcping motor system. Ann.Neurol 30:476,1991.

Lord,J. Cerebral palsy:A clinical approach. Arch.Phys.Med.Rehabil. $65: 542-548,1984$.

Louis,D.S. Greene,T.L. Jacobson,K.E. Rasmussen,C. Kolowich,P. and Goldstein,S.A. Evaluation of normal values for stationary and moving two-point discrimination in the hand. JHand Surg 9:552-555,1984.

Majnemer,A. Rosenblatt,B. Riley,P. Laureau,E. and O'Gorman,A.M. Somatosensory evoked response abnormalities in high-risk newborns. Pediatr.Neurol, 3:350-355,1987.

Majnemer,A. Rosenblatt,B. and Riley,P. Prognostic significance of the auditory brainstem evoked response in high-risk roonates. Dev.Med.Child Neurol, 30:43-52,1988. 
Martin,J.H. and Jessel,T.M. Anatomy of the somatic sensory system. In:Principles of Neural Science. Edited by Kandel,E.R., Schwartz,J.H. and Jessel,T.M. 3rd edition,. New York:Elsevier Science Publishing company, 1991,pp.353-366.

Mathiowetz,V. Kashman,N. Volland,G. Weber,K. Dowe,M. and Rogers,S. Grip and pinch strength:Normative data for adults. Arch.Phys.Med.Rehabil, 66:69-74,1985.

Mathiowetz,V. Weber,K. Volland,G. and Kashman,N. Reliabılity and validity of grip and pinch strength evaluations. J.Hand Surg. 9:222226,1984 .

Mathiowetz, V. Wieman,D. and Federman,S. Grip and pinch strength:norms for 6-19 year olds. Am.J.Occup.Ther, 40:705711,1986 .

Maugiere,F. Desmedt,J. and Courjon,J. Astereognosis and dissociated loss of frontal or parietal components of somatosensory evoked potentials in hemispheric lesions. Brain. 106:271-311,1983.

Miller,L.J. Miller Assessment for Preschoolers (MAP). Colorado:Foundation for the Knowledge in Development. Copyright, 1982.

Minear,W. Classification of cerebral palsy. Pediatr 18:841,1956.

Monfraix,C. Tardieu,G. and Tardieu,C. Disturbances of manual perception in children with cerebral palsy. Cereb.Palsy Bull.3. 6:544$552,1961$.

Monfraix,C. and Tardieu,G. Development of manual perception in the child with cerebral palsy during re-education. Cereb.Palsy Bull3. 6:553-558,1961. 
Moore,J.C. Recovery potentials following CNS lesions:A brief historical perspective in relation to modern research data on neuroplasticity. Am.J.Occup. Ther, 40:459-463,1986.

Mountcastle,V.B. (Ed.) Sensory receptors and neural encoding:introduction to sensory processes. In:Medical Physiology 14th edition. Toronto:C.V.Mosby Co. 1980,pp.327-347.

Mutti,M. Sterling,H.M. and Spalding,N.V. The Quick Neurological Screening Test (ONST). California:Western Psychological Services (WPS), Copyright by Academic Therapy Publications Inc., 1978.

Nass,R. and Koch,D. Innate specialization for emotion:Temperament differences in children with early left versus right brain damage. PediatrAdolesc.Med 1:1-17,1991.

Nolan,M.F. Limits of two-point discrimination ability in the lower limb in young adult men and women. J.Am.Phys. Ther.Assoc. 63:1424$1428,1983$.

Norsell,U. Behavioral studies of the somatosensory system. Physiol.Rev, 60:327-354,1980.

Olson,C.L. (Ed.) Measures of dispersion. In:Statistics:Making sense of data. Toronto:Allyn and Bacon Inc., 1987,pp.122-126.

Opila-Lehman,J., Short,M.A. and Trombly,C.A. Kinesthetic recall of children with athetoid and spastic cerebral palsy and of nonhandicapped children. Dev.Med.Child Neurol. 27:223-230,1985.

Paul,R.L. Merzenich,M. and Goodman,H. Representation of slowly and rapidly adapting cutaneous mechanoreceptors of the hand in Broadmann's areas 3 and 1 of Macaca Mulatta. Brain Res. 36:229249,1972 . 
Pause,M. and Freund,H.J. Role of the parietal cortex for sensorimotor transformation. Brain Behav.Evol 33:136-140,1989.

Randolph,M. and Semmes,J. Behavioral consequences of selective subtotal ablations in the postcentral gyrus of Macaca Mulatta. Brain Res. 70:55-70,1974.

Ridley,R.M. and Ettlinger,G. Impaired tactile learning and retention after removal of the second somatic sensory projective cortex (SII) in the monkey. Brain Res.109:656-660,1976.

Rogers,J.C. Selection of evaluation instruments. In:A Therapist's Guide to pediatric Assessment Edited by King-Thomas,L. and Hacker,B.J. Toronto:Little, Brown and Company, 1987,pp.19-34.

Roland,P.E. Astereognosis. Tactile discrimination after localized hemispheric lesions in man. Arch.Neurol 33:543-550,1976.

Roland,P.E. Somatosensory detection of microgeometry, macrogeometry and kinesthesia after localized lesions of the cerebral hemispheres in man. Brain Res.Rev 12:43-94,1987.

Ruch,T.C. Fulton,J.F. and German,W.J. Sensory discrimination in monkey, chimpanzee and man after lesions of the parietal lobe. Arch.Neurol.Psychiatr, 39:919-938,1938.

Ryerson,S.D. Hemiplegia resulting from vascular insult or disease. In:Neurological Rehabilitation. Edited by Umphred,D.A. 2nd edition, Toronto:C.V.Mosby Co., 1990,pp.619.

Scott,A.D. and Trombly,C.A. Evaluation. Occupational Therapy for Physical Dysfunction. Edited by Trombly,C.A. 3rd edition. Baltimore:Williams and Wilkins, 1989,pp.184-218. 
Seal,J. Sensory and motor functions of the superior parietal cortex of the monkey as revealed by single-neuron recordings. Brain Beh.Evol 33:113-117,1989.

Semmes,J. and Porter,L. A comparison of precentral and postcentral cortical lesions on somatosensory discrimination in the monkey. Cortex, 8:249-264,1972.

Semmes,J. and Turner,B. Effects of cortical lesions on somatosensory tasks. Junvestigative Dermatology 69:181$189,1977$.

Semmes,J. Weinstein,S. Ghent,L. and Teuber,H-L. Normative study. In:Sumatesensory changes after penetrating brain wounds in man Edited by Semmes,J. Cambridge:Harvard University Press, 1960,pp.411.

Skerik,S. Weiss,M. and Flatt,A. Functional evaluation of congenital hand anomalies. Part I. Am.J.Occup.Ther. 25:98-104,1971.

Stowers,S. and Huber,C.J. Developmental and screening tests. In:A Iherapist's Guide to pediatric Assessment Edited by King-Thomas,L. and Hacker,B.J. Toronto:Little, Brown and Company, 1987,pp.43-75.

Tachdjian,M.O. and Minear,W.L. Sensory disturbances in the hands of children with cerebral palsy. LBone Joint Sura 40:85-90,1958.

Tardieu,G. Some questions and answers regarding astereognosis in cerebral palsy. Clin.Orthoe 46:55-81,1966.

Taylor,M and Fagan,E. Somatosensory evoked potentials to median nerve stimulation:normative data for paediatrics. Electroencephalogr.Clin.Neurophysiol. 71:323-330,1988.

Tizard,J. Paine,R.S. and Crothers,B. Disturbances of sensation in children with hemiplegia. J.A.M.A. 155:628-632,1954. 
Tizard,J. and Crothers,B. Sensory disturbances in hemiplegia in childhood. Irans.Am.Neurol.Assoc. 77:227-229,1952.

Twitchell,T.E. Sensation and the motor deficit in cerebral palsy. Clin.Orthor 46:55-81,1966.

Twitchell,T.E. The grasping deficit in infantile spastic hemiparesis. Neurology, 8:13-21,1958.

Van Buskirk,C. and Webster,D. Prognostic value of sensory defect in rehabilitation of hemiplegics. Neurology 5:407-411,1955.

Volpe,J.J. (Ed.) Hypoxic-ischiamir, encephalopathy In:Neurology of the newborn. 2nd edition, Philadelphia:W.D.Saunders Company, 1987,pp.159-279.

Volpe,J.J. (Ed.) Intracranial hemorrhage In:Neurology of the newborn. 2nd edition, Philadelphia:W.D.Saunders Company, 1987,pp.281-361.

Volpe,J.J. (Ed.) Metabolic encephalopathies In:Neurology of the newborn. 2nd edition, Philadelphia:W.D.Saunders Company, 1987,pp.363-408.

Waylett-Rendall,J. Sensibility evaluation and rehabilitation. Orthop.Clin.North Am. 19:43-56,1988.

Weiss, M. and Flatt,A. Functional evaluation of the congenitally anomalous hand. Part II. Am.J.Occup. Ther. 25:139-143,1971.

Westling,G. and Johansson,R. Factors influencing the force control during precision grip. Exp.Brain Research 53:277-284,1984.

Williamson,P. Goff,W. and Allison, T. Somato-sensory evoked responses in patients with unilateral cerebral lesions. Electroencephaloar.Clin.Neurophysiol. 28:566-575,1970. 
Wong,P. Lombroso,C. and Matsumiya,Y. Somatosensory evoked potentials:Variability analysis in unilateral hemispheric disease. Electroencephaloar.Clin.Neurophysiol, 54:266-274,1982.

Zeman,B. and Yiannikas,C. Functional prognosis in stroke:use of somatosensory evoked potentials. LNeurolNeurosurg.Psychiatr $52: 242-247,1989$.

Zhu,Y. Georgesco,M. and Cadihac,J. Normal latency values of early cortical somatosensory evoked potentials in children. Electroencephaloar.Clin.Neurophysiol. 68:471-474,1987. 